\title{
The exit from a metastable state: Concentration of the exit point distribution on the low energy saddle points, part 1
}

\author{
Giacomo Di Gesù ${ }^{\mathrm{a}, \mathrm{b}}$, Tony Lelièvre ${ }^{\mathrm{b}, *}$, Dorian Le Peutrec ${ }^{\mathrm{c}}$, Boris Nectoux ${ }^{\mathrm{a}, \mathrm{b}}$ \\ a Institut für Analysis und Scientific Computing, E101-TU Wien, Wiedner Hauptstr. 8, 1040 Wien, \\ Austria \\ b CERMICS, École des Ponts, Université Paris-Est, INRIA, 77455 Champs-sur-Marne, France \\ c Laboratoire de Mathématiques d'Orsay, Univ. Paris-Sud, CNRS, Université Paris-Saclay, 91405 Orsay, \\ France
}

MSC:
60J60
58J65
35Q82
58C40
81Q20

Keywords:

Overdamped Langevin

Exit problem

Small temperature regime

Semi-classical analysis

\section{A B S T R A C T}

We consider the first exit point distribution from a bounded domain $\Omega$ of the stochastic process $\left(X_{t}\right)_{t \geq 0}$ solution to the overdamped Langevin dynamics

$$
d X_{t}=-\nabla f\left(X_{t}\right) d t+\sqrt{h} d B_{t}
$$

starting from the quasi-stationary distribution in $\Omega$. In the small temperature regime $(h \rightarrow 0)$ and under rather general assumptions on $f$ (in particular, $f$ may have several critical points in $\Omega$ ), it is proven that the support of the distribution of the first exit point concentrates on some points realizing the minimum of $f$ on $\partial \Omega$. Some estimates on the relative likelihood of these points are provided. The proof relies on tools from semi-classical analysis.

R É S U M É

Dans ce travail, nous étudions la distribution du point de sortie d'un domaine borné $\Omega$ pour le processus stochastique $\left(X_{t}\right)_{t \geq 0}$ solution de la dynamique de Langevin suramortie

$$
d X_{t}=-\nabla f\left(X_{t}\right) d t+\sqrt{h} d B_{t}
$$

initialement distribué suivant la distribution quasi-stationnaire dans $\Omega$. Dans la limite basse température $h \rightarrow 0$ et sous des hypothèses générales sur la fonction $f$ ( $f$ pouvant notamment avoir plusieurs points critiques dans $\Omega$ ), nous montrons que la distribution du lieu de sortie se concentre sur certains points réalisant le minimum de $f$ sur $\partial \Omega$. Nous calculons aussi les probabilités relatives de sortir autour de chacun de ces points. Nos preuves reposent sur des outils issus de l'analyse semi-classique.

* Corresponding author.

E-mail addresses: giacomo.di.gesu@asc.tuwien.ac.at (G. Di Gesù), tony.lelievre@enpc.fr (T. Lelièvre), dorian.lepeutrec@math.u-psud.fr (D. Le Peutrec), boris.nectoux@asc.tuwien.ac.at (B. Nectoux). 


\section{Introduction and main results}

\subsection{Setting and motivation}

We are interested in the overdamped Langevin dynamics

$$
d X_{t}=-\nabla f\left(X_{t}\right) d t+\sqrt{h} d B_{t}
$$

where $X_{t}$ is a vector in $\mathbb{R}^{d}, f: \mathbb{R}^{d} \rightarrow \mathbb{R}$ is a $C^{\infty}$ function, $h$ is a positive parameter and $\left(B_{t}\right)_{t \geq 0}$ is a standard $d$-dimensional Brownian motion. Such a dynamics is prototypical of models used for example in computational statistical physics to simulate the evolution of a molecular system at a fixed temperature, in which case $f$ is the potential energy function and $h$ is proportional to the temperature. It admits as an invariant measure the Boltzmann-Gibbs measure (canonical ensemble) $Z^{-1} e^{-\frac{2}{h} f(x)} d x$ where $Z=\int_{\mathbb{R}^{d}} e^{-\frac{2}{h} f}<\infty$. In the small temperature regime $h \rightarrow 0$, the stochastic process $\left(X_{t}\right)_{t \geq 0}$ is typically metastable: it stays for a very long period of time in a neighborhood of a local minimum of $f$ (called a metastable state) before hopping to another metastable state. In the context of statistical physics, this behavior is expected since the molecular system typically jumps between various conformations, which are indeed these metastable states. For modeling purposes as well as for building efficient numerical methods (see for instance [1-3]), it is thus interesting to be able to precisely describe the exit event from a metastable state, namely the law of the first exit time and the first exit point.

The main objective of this work is to address the following question: given a metastable domain $\Omega \subset \mathbb{R}^{d}$, what are the exit points in the small temperature regime $h \rightarrow 0$ ? This is formalized mathematically by the notion of concentration, which is now introduced. For a domain $\Omega \subset \mathbb{R}^{d}$ and a given initial condition $X_{0}$, let us consider the exit event $\left(\tau_{\Omega}, X_{\tau_{\Omega}}\right)$ from $\Omega$ where

$$
\tau_{\Omega}=\inf \left\{t \geq 0 \mid X_{t} \notin \Omega\right\}
$$

is the first exit time from $\Omega$. We will consider the family of laws of $X_{\tau_{\Omega}}$, as $h$ goes to zero, and prove that these distributions concentrate on a subset of $\partial \Omega$, as defined below.

Definition 1. Let $\mathcal{Y} \subset \partial \Omega$ and let us consider a family of random variables $\left(Y_{h}\right)_{h \geq 0}$ which admits a limit in distribution when $h \rightarrow 0$. The law of $Y_{h}$ concentrates on $\mathcal{Y}$ in the limit $h \rightarrow 0$ if for every neighborhood $\mathcal{V}_{\mathcal{Y}}$ of $\mathcal{Y}$ in $\partial \Omega$,

$$
\lim _{h \rightarrow 0} \mathbb{P}\left[Y_{h} \in \mathcal{V}_{\mathcal{Y}}\right]=1
$$

and if for all $x \in \mathcal{Y}$ and for all neighborhoods $\mathcal{V}_{x}$ of $x$ in $\partial \Omega$,

$$
\lim _{h \rightarrow 0} \mathbb{P}\left[Y_{h} \in \mathcal{V}_{x}\right]>0
$$

In other words, $\mathcal{Y}$ is the support of the law of $Y_{h}$ in the limit $h \rightarrow 0$.

Previous results on the behavior of the law of $X_{\tau_{\Omega}}$ when $h \rightarrow 0$. Let us report on previous results on the law of $X_{\tau_{\Omega}}$ in the limit $h \rightarrow 0$ (see also [4] for a comprehensive review of the literature).

First, some results have been obtained using formal asymptotic expansions. For example, in [5], the concentration of the law of $X_{\tau_{\Omega}}$ on $\arg \min _{\partial \Omega} f$ in the small temperature regime $(h \rightarrow 0)$ has been studied when $\partial_{n} f>0$ on $\partial \Omega$ (here and in the following, $\partial_{n} f$ is the outward normal derivative of $f$ on $\partial \Omega$ ). See also $[6,7]$ for generalizations to the case $\partial_{n} f=0$ on $\partial \Omega$. 
Using partial differential equation techniques, some of the formal results above have been rigorously proven. For example, when

$$
\partial_{n} f>0 \text { on } \partial \Omega
$$

and

$$
\{x \in \Omega,|\nabla f(x)|=0\}=\left\{x_{0}\right\} \text { with } f\left(x_{0}\right)=\min _{\bar{\Omega}} f \text { and } \operatorname{det} \operatorname{Hess} f\left(x_{0}\right)>0,
$$

the concentration of the law of $X_{\tau_{\Omega}}$ in the limit $h \rightarrow 0$ on $\arg \min _{\partial \Omega} f$ has been obtained in [8-10], when $X_{0}=x \in \Omega$, see also [11,12] for more recent results with similar techniques.

Finally, another rigorous approach to study the exit point distribution is to rely on the theory of large deviations. When (3)-(4) hold and $f$ attains its minimum on $\partial \Omega$ at a single point $y_{0}$, [13, Theorem 2.1 in Chapter 4.2] implies that the law of $X_{\tau_{\Omega}}$ concentrates on $y_{0}$ in the limit $h \rightarrow 0$, when $X_{0}=x \in \Omega$. This result has then been generalized in [14,15] when only (3)-(4) are satisfied. In [13, Theorem 5.1 in Chapter 6.5], under more general assumptions on $f$, for $\Sigma \subset \partial \Omega$, the limit of $h \ln \mathbb{P}\left[X_{\tau_{\Omega}} \in \Sigma\right]$ when $h \rightarrow 0$ is related to a minimization problem involving the quasipotential of the process (1). Let us mention two limitations when applying [13, Theorem 5.1 in Chapter 6.5] in order to obtain some information on the first exit point distribution. First, this theorem requires to be able to compute the quasipotential in order to get useful information: this is trivial under the assumptions (3)-(4) but more complicated for a general function $f$ (in particular when $f$ has several critical points in $\Omega$ ). Second, even when the quasipotential is analytically known, this result only gives the subset of $\partial \Omega$ through which exit occurs with an exponentially small probability in the limit $h \rightarrow 0$. It does not allow to exclude points through which exit occurs with a polynomially small probability in $h$ for example (this indeed happens, see Section 1.4). Besides, it does not give the relative probability of the exit points which have a non-zero probability in the limit $h \rightarrow 0$.

The quasi-stationary distribution approach which is used in this work to study the exit event has been introduced in $[16,17,1]$. Notice that compared to the work [1], we here only identify the support of the first exit point distribution, and the relative likelihood of the points in this support, whereas in [1], we also study the exit through points which occur with exponentially small probability in the limit $h \rightarrow 0$. The results here are thus less precise than in [1], but the assumptions on $f$ are also much more general.

To conclude this short review of the literature, let us mention that $[8-10,14,15,13]$ also cover the case of non reversible diffusions, while we here only consider the reversible dynamics (1).

Purpose of this work: a general geometric setting and a precise description of the exit point distribution. In this work, we study the concentration of the law of $X_{\tau_{\Omega}}$ on $\arg \min _{\partial \Omega} f$ in the limit $h \rightarrow 0$. Compared to results previously obtained in the literature, the novelty is twofold: first, the geometric setting is much more general, and second, we obtain a precise description of the first exit point distribution, by providing the relative probabilities of the exit points.

More precisely, we exhibit assumptions on $f$ which ensure that when $X_{0}$ is distributed according to the quasi-stationary distribution $\nu_{h}$ in $\Omega$ (see Definition 2 below), the law of $X_{\tau_{\Omega}}$ concentrates in the limit $h \rightarrow 0$ on some global minima of $f$ on $\partial \Omega$ : these global minima and the relative probability of these exit points are made explicit. The geometric setting is much more general than (3)-(4). For instance, it is not assumed that $\partial_{n} f>0$ on $\partial \Omega$, there is no restriction on the number of critical points of $f$ in $\Omega$ and $f$ is allowed to have critical points in $\Omega$ with larger energies than $\min _{\partial \Omega} f$ (however we do not consider the case when $f$ has critical points on $\partial \Omega$, and we work under some Morse assumption on $f$ ).

Let us emphasize that this work requires that $X_{0}$ is distributed according to the quasi-stationary distribution $\nu_{h}$ in $\Omega$ (see Definition 2 below). This is relevant when the exit from the domain $\Omega$ is metastable, namely when the process (1) reaches a local equilibrium within $\Omega$ before exiting $\Omega$ (see Section 1.2 below 
and [1-3]). The companion paper [18] builds on and extends the result of the present work to general initial conditions in $\Omega$, as explained in Remark 8 below.

Here are representative examples of outputs of this work. First, if $\left\{y \in \Omega, f(y)<\min _{\partial \Omega} f\right\}$ is connected and contains all the critical points of $f$ in $\Omega$, and if $\partial_{n} f>0$ on $\arg \min _{\partial \Omega} f$, then the law of $X_{\tau_{\Omega}}$ concentrates on $\arg \min _{\partial \Omega} f$ when $X_{0} \sim \nu_{h}$. Besides, when some critical points of $f$ in $\Omega$ are larger in energy than $\min _{\partial \Omega} f$, then the law of $X_{\tau_{\Omega}}$ concentrates on a subset of $\partial \Omega$ which may be strictly included in $\arg \min _{\partial \Omega} f$. In particular, the following phenomena may occur:

(i) There may exist points $z \in \arg \min _{\partial \Omega} f, C>0$ and $c>0$, such that for every sufficiently small neighborhood $\Sigma_{z}$ of $z$ in $\partial \Omega$, in the limit $h \rightarrow 0, \mathbb{P}_{\nu_{h}}\left[X_{\tau_{\Omega}} \in \Sigma_{z}\right] \leq C e^{-\frac{c}{h}}$ (see (24) in Theorem 1 and the discussion after the statement of Theorem 1 ).

(ii) There may exist points $z \in \arg \min _{\partial \Omega} f$ and $C>0$ such that for every sufficiently small neighborhood $\Sigma_{z}$ of $z$ in $\partial \Omega, \mathbb{P}_{\nu_{h}}\left[X_{\tau_{\Omega}} \in \Sigma_{z}\right]=C \sqrt{h}(1+o(1))$. This is explained in Section 1.4.

Let us finally mention that while proving these results, we also obtain a sharp asymptotic estimate on the principal eigenvalue (when $h \rightarrow 0$ ) and on the principal eigenfunction of the infinitesimal generator of the diffusion (1) with Dirichlet boundary conditions on $\partial \Omega$, see Section 1.3.4. These results have their own interests.

Organization of the end of Section 1. In Section 1.2, the quasi-stationary distribution of the process (1) in $\Omega$ is introduced. In Section 1.3, the assumptions on $f$ which will be used throughout this paper are presented and the main result of this work is stated (see Theorem 1). Finally, the necessity of the assumptions of Theorem 1 is discussed in Section 1.4.

\subsection{Metastability and the quasi-stationary distribution}

The quasi-stationary distribution is the cornerstone of our analysis. Here, we assume that $\Omega \subset \mathbb{R}^{d}$ is smooth, open, bounded and connected (see Section 1.3 for the general geometric setting).

Definition 2. A quasi-stationary distribution for the stochastic process $\left(X_{t}\right)_{t \geq 0}$ in the domain $\Omega \subset \mathbb{R}^{d}$ is a probability measure $\nu_{h}$ supported in $\Omega$ such that for all measurable sets $A \subset \Omega$ and for all $t \geq 0$

$$
\nu_{h}(A)=\frac{\int_{\Omega} \mathbb{P}_{x}\left[X_{t} \in A, t<\tau_{\Omega}\right] \nu_{h}(d x)}{\int_{\Omega} \mathbb{P}_{x}\left[t<\tau_{\Omega}\right] \nu_{h}(d x)} .
$$

Here and in the following, the subscript $x$ indicates that the stochastic process starts from $x \in \mathbb{R}^{d}$ $\left(X_{0}=x\right)$. In words, (5) means that if $X_{0}$ is distributed according to $\nu_{h}$, then for all $t>0, X_{t}$ is still distributed according to $\nu_{h}$ conditionally on $X_{s} \in \Omega$ for all $s \in[0, t]$. The following results have been proven in [16] (see also [19] for much more general results on quasi-stationary distributions):

Proposition 3. Let $\Omega \subset \mathbb{R}^{d}$ be a bounded domain and consider the dynamics (1). Then there exists a probability measure $\nu_{h}$ with support in $\Omega$ such that, whatever the law of the initial condition $X_{0}$ with support in $\Omega$, it holds:

$$
\lim _{t \rightarrow \infty}\left\|\operatorname{Law}\left(X_{t} \mid t<\tau_{\Omega}\right)-\nu_{h}\right\|_{T V}=0
$$


Here, $\operatorname{Law}\left(X_{t} \mid t<\tau_{\Omega}\right)$ denotes the law of $X_{t}$ conditional to the event $\left\{t<\tau_{\Omega}\right\}$. A corollary of this proposition is that the quasi-stationary distribution $\nu_{h}$ exists and is unique. For a given initial distribution of the process (1), if the convergence in (6) is much quicker than the exit from $\Omega$, the exit from the domain $\Omega$ is said to be metastable. When the exit from $\Omega$ is metastable, it is thus relevant to study the exit event from $\Omega$ assuming that the process (1) is initially distributed according to the quasi-stationary distribution $\nu_{h}$. This will be the setting of this work.

Let us now relate the notion of quasi-stationary distribution to the infinitesimal generator of the dynam$\operatorname{ics}(1)$

$$
L_{f, h}^{(0)}=-\nabla f \cdot \nabla+\frac{h}{2} \Delta .
$$

In the notation $L_{f, h}^{(0)}$, the superscript (0) indicates that we consider an operator on functions, namely 0-forms. The basic observation to define our functional framework is that the operator $L_{f, h}^{(0)}$ is self-adjoint on the weighted $L^{2}$ space

$$
L_{w}^{2}(\Omega)=\left\{u: \Omega \rightarrow \mathbb{R}, \int_{\Omega} u^{2} e^{-\frac{2}{h} f}<\infty\right\}
$$

(the weighted Sobolev spaces $H_{w}^{k}(\Omega)$ are defined similarly). Indeed, for any smooth test functions $u$ and $v$ with compact supports in $\Omega$, one has

$$
\int_{\Omega}\left(L_{f, h}^{(0)} u\right) v e^{-\frac{2}{h} f}=\int_{\Omega}\left(L_{f, h}^{(0)} v\right) u e^{-\frac{2}{h} f}=-\frac{h}{2} \int_{\Omega} \nabla u \cdot \nabla v e^{-\frac{2}{h} f} .
$$

This gives a proper framework to introduce the Dirichlet realization $L_{f, h}^{D,(0)}$ on $\Omega$ of the operator $L_{f, h}^{(0)}$ as follows:

Proposition 4. The Friedrichs extension associated with the quadratic form $\phi \in C_{c}^{\infty}(\Omega) \mapsto \frac{h}{2} \int_{\Omega}|\nabla \phi|^{2} e^{-\frac{2}{h} f} i s$ denoted by $-L_{f, h}^{D,(0)}$. It is a non negative unbounded self-adjoint operator on $L_{w}^{2}(\Omega)$ with domain $D\left(L_{f, h}^{D,(0)}\right)$ $=H_{w, 0}^{1}(\Omega) \cap H_{w}^{2}(\Omega)$, where $H_{w, 0}^{1}(\Omega)=\left\{u \in H_{w}^{1}(\Omega), u=0\right.$ on $\left.\partial \Omega\right\}$.

The compact injection $H_{w}^{1}(\Omega) \subset L_{w}^{2}(\Omega)$ implies that the operator $L_{f, h}^{D,(0)}$ has a compact resolvent and its spectrum is consequently purely discrete. Let us introduce $\lambda_{h}>0$ the smallest eigenvalue of $-L_{f, h}^{D,(0)}$ (a.k.a. the principal eigenvalue):

$$
\lambda_{h}=\inf \sigma\left(-L_{f, h}^{D,(0)}\right) .
$$

From standard results on elliptic operators (see e.g. [20,21]), $\lambda_{h}$ is non degenerate and its associated eigenfunction $u_{h}$ has a sign on $\Omega$. Moreover, $u_{h} \in C^{\infty}(\bar{\Omega})$. Without loss of generality, one can then assume that:

$$
u_{h}>0 \text { on } \Omega \text { and } \int_{\Omega} u_{h}^{2} e^{-\frac{2}{h} f}=1 .
$$

The eigenvalue-eigenfunction pair $\left(\lambda_{h}, u_{h}\right)$ satisfies:

$$
\left\{\begin{aligned}
-L_{f, h}^{(0)} u_{h} & =\lambda_{h} u_{h} \text { on } \Omega \\
u_{h} & =0 \text { on } \partial \Omega
\end{aligned}\right.
$$


The link between the quasi-stationary distribution $\nu_{h}$ and the function $u_{h}$ is given by the following proposition (see for example [16]):

Proposition 5. The unique quasi-stationary distribution $\nu_{h}$ associated with the dynamics (1) and the domain $\Omega$ is given by:

$$
\nu_{h}(d x)=\frac{u_{h}(x) e^{-\frac{2}{h} f(x)}}{\int_{\Omega} u_{h}(y) e^{-\frac{2}{h} f(y)} d y} d x .
$$

Let us recall that $\partial_{n}=n \cdot \nabla$ stands for the normal derivative and $n$ is the unit outward normal on $\partial \Omega$. The next proposition (see again [16]) characterizes the law of the exit event from $\Omega$.

Proposition 6. Let us consider the dynamics (1) and the quasi-stationary distribution $\nu_{h}$ associated with the domain $\Omega$. If $X_{0}$ is distributed according to $\nu_{h}$, the random variables $\tau_{\Omega}$ and $X_{\tau_{\Omega}}$ are independent. Furthermore $\tau_{\Omega}$ is exponentially distributed with parameter $\lambda_{h}$ and the law of $X_{\tau_{\Omega}}$ has a density with respect to the Lebesgue measure on $\partial \Omega$ given by

$$
z \in \partial \Omega \mapsto-\frac{h}{2 \lambda_{h}} \frac{\partial_{n} u_{h}(z) e^{-\frac{2}{h} f(z)}}{\int_{\Omega} u_{h}(y) e^{-\frac{2}{h} f(y)} d y} .
$$

\subsection{Hypotheses and main results}

\subsubsection{Hypotheses and notation}

In the following, we consider a setting that is actually more general than the one of Section 1.2: $\bar{\Omega}$ is a $C^{\infty}$ oriented compact and connected Riemannian manifold of dimension $d$ with boundary $\partial \Omega$.

The following notation will be used: for $a \in \mathbb{R},\{f<a\}=\{x \in \bar{\Omega}, f(x)<a\},\{f \leq a\}=\{x \in \bar{\Omega}, f(x) \leq$ $a\}$ and $\{f=a\}=\{x \in \bar{\Omega}, f(x)=a\}$. Let us now introduce the basic assumption on $f$ which is used throughout this work:

The function $f: \bar{\Omega} \rightarrow \mathbb{R}$ is $C^{\infty}$, and for all $x \in \partial \Omega,|\nabla f(x)| \neq 0$.

The functions $f: \Omega \rightarrow \mathbb{R}$ and $f:\left\{x \in \partial \Omega, \partial_{n} f(x)>0\right\} \rightarrow \mathbb{R}$ are Morse.

Moreover, $f$ has at least one local minimum in $\Omega$.

Let us recall that a function $\phi: \bar{\Omega} \rightarrow \mathbb{R}$ is a Morse function if all its critical points are non degenerate (which implies in particular that $\phi$ has a finite number of critical points since $\bar{\Omega}$ is compact and a non degenerate critical point is isolated from the other critical points). A critical point $z \in \bar{\Omega}$ of $\phi$ is non degenerate if the Hessian matrix of $\phi$ at $z$, denoted by Hess $\phi(z)$, is invertible. We refer for example to [22, Definition 4.3.5] for a definition of the Hessian matrix on a manifold. A non degenerate critical point $z \in \bar{\Omega}$ of $\phi$ is said to have index $p \in\{0, \ldots, d\}$ if Hess $\phi(z)$ has precisely $p$ negative eigenvalues (counted with multiplicity). In the case $p=1, z$ is called a saddle point.

For any local minimum $x$ of $f$ in $\Omega$, the height of the energy barrier to leave $\Omega$ from $x$ is

$$
\mathbf{H}_{f}(x):=\inf _{\substack{\gamma \in C^{0}([0,1], \bar{\Omega}) \\ \gamma(0)=x, \gamma(1) \in \partial \Omega}} \max _{t \in[0,1]} f(\gamma(t)),
$$

where $C^{0}([0,1], \bar{\Omega})$ is the set of continuous paths from $[0,1]$ to $\bar{\Omega}$. Let us now define a set of assumptions which will be used below: 
- (A0) holds and

$$
\exists ! C_{\max } \in \mathcal{C} \text { such that } \max _{\mathcal{C} \in \mathcal{C}}\left\{\max _{\overline{\bar{C}}} f-\min _{\overline{\mathrm{C}}} f\right\}=\max _{\overline{\mathrm{C}_{\max }}} f-\min _{\overline{\mathrm{C}_{\max }}} f
$$

where

$$
\mathcal{C}:=\{\mathrm{C}(x), x \text { is a local minimum of } f \text { in } \Omega\},
$$

with, for any local minimum $x$ of $f$ in $\Omega$,

$$
\mathrm{C}(x) \text { is the connected component of }\left\{f<\mathrm{H}_{f}(x)\right\} \text { containing } x \text {. }
$$

- (A1) holds and

$$
\partial \mathrm{C}_{\max } \cap \partial \Omega \neq \emptyset .
$$

- (A1) holds and

$$
\partial \mathrm{C}_{\max } \cap \partial \Omega \subset \underset{\partial \Omega}{\arg \min } f .
$$

It will be shown that the assumptions (A0), (A1), (A2), and (A3) ensure that when $X_{0} \sim \nu_{h}$, the law of $X_{\tau_{\Omega}}$ concentrates on the set $\partial \mathrm{C}_{\max } \cap \partial \Omega$, see items 1 and 2 in Theorem 1. Finally, let us introduce assumption (A4):

- (A1) holds and

$$
\partial \mathrm{C}_{\max } \cap \Omega \text { contains no separating saddle point of } f \text {. }
$$

Roughly speaking, a saddle point $z$ of $f$ is separating if for any sufficiently small connected neighborhood $\mathcal{V}_{z}$ of $z, \mathcal{V}_{z} \cap\{f<f(z)\}$ has two connected components included in two different connected components of $\{f<f(z)\}$. We refer to Definition 13 below for more details. The assumption (A4) together with (A0), (A1), (A2), and (A3), ensures that the probability that the process (1) leaves $\Omega$ through any sufficiently small neighborhood of $z \in \partial \Omega \backslash \partial \mathrm{C}_{\max }$ in $\partial \Omega$ is exponentially small when $h \rightarrow 0$, see item 3 in Theorem 1.

Fig. 1 gives a one-dimensional example where (A1), (A2), (A3) and (A4) are satisfied. In Section 1.4, the necessity of assumptions (A1), (A2), (A3), and (A4) is discussed. We will actually work with equivalent formulations of the assumptions (A1), (A2), (A3), and (A4) which will be given in Section 2.4.

\subsubsection{Notation for the local minima and saddle points of the function $f$}

The main purpose of this section is to introduce the local minima and the generalized saddle points of $f$. These elements of $\bar{\Omega}$ are used extensively throughout this work and play a crucial role in our analysis. Roughly speaking, the generalized saddle points of $f$ are the saddle points $z \in \bar{\Omega}$ of the function $f$ extended by $-\infty$ outside $\bar{\Omega}$ (which is indeed consistent with the homogeneous Dirichlet boundary conditions in (10)). Thus, when the function $f$ satisfies the assumption (A0), a generalized saddle point of $f$ (as introduced in [23]) is either a saddle point $z \in \Omega$ of $f$ or a local minimum $z \in \partial \Omega$ of $\left.f\right|_{\partial \Omega}$ such that $\partial_{n} f(z)>0$.

Let us assume that the function $f$ satisfies the assumption (A0). Let us denote by

$$
\mathrm{U}_{0}^{\Omega}=\left\{x_{1}, \ldots, x_{\mathrm{m}_{0}^{\Omega}}\right\} \subset \Omega
$$




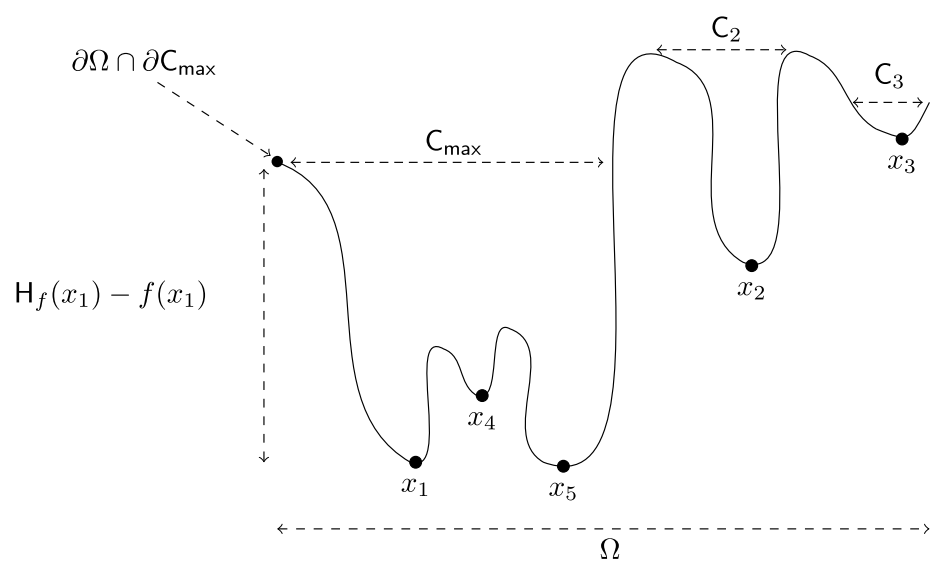

Fig. 1. A one-dimensional case where (A1), (A2), (A3) and (A4) are satisfied. On the figure, $f\left(x_{1}\right)=f\left(x_{5}\right), \mathbf{H}_{f}\left(x_{1}\right)=\mathrm{H}_{f}\left(x_{4}\right)=$ $\mathrm{H}_{f}\left(x_{5}\right), \mathcal{C}=\left\{\mathrm{C}_{\max }, \mathrm{C}_{2}, \mathrm{C}_{3}\right\}, \partial \mathrm{C}_{2} \cap \partial \mathrm{C}_{\max }=\emptyset$ and $\partial \mathrm{C}_{3} \cap \partial \mathrm{C}_{\max }=\emptyset$.

the set of local minima of $f$ in $\Omega$ where $\mathrm{m}_{0}^{\Omega} \in \mathbb{N}$ is the number of local minima of $f$ in $\Omega$. Notice that since $f$ satisfies $(\mathrm{A} 0), \mathrm{m}_{0}^{\Omega} \geq 1$. The set of saddle points of $f$ of index $1 \mathrm{in} \Omega$ is denoted by $\mathrm{U}_{1}^{\Omega}$ and its cardinality by $\mathrm{m}_{1}^{\Omega}$. Let us define

$$
\mathrm{U}_{1}^{\partial \Omega}:=\left\{z \in \partial \Omega, z \text { is a local minimum of }\left.f\right|_{\partial \Omega} \text { but not a local minimum of } f \text { in } \bar{\Omega}\right\}
$$

Notice that an equivalent definition of $U_{1}^{\partial \Omega}$ is

$$
\mathrm{U}_{1}^{\partial \Omega}=\left\{z \in \partial \Omega, z \text { is a local minimum of }\left.f\right|_{\partial \Omega} \text { and } \partial_{n} f(z)>0\right\}
$$

which follows from the fact that $\nabla f(x) \neq 0$ for all $x \in \partial \Omega$. Let us introduce

$$
\mathrm{m}_{1}^{\partial \Omega}:=\operatorname{Card}\left(\mathrm{U}_{1}^{\partial \Omega}\right)
$$

In addition, one defines:

$$
\mathrm{U}_{1}^{\bar{\Omega}}:=\mathrm{U}_{1}^{\partial \Omega} \cup \mathrm{U}_{1}^{\Omega} \text { and } \mathrm{m}_{1}^{\bar{\Omega}}:=\operatorname{Card}\left(\mathrm{U}_{1}^{\bar{\Omega}}\right)=\mathrm{m}_{1}^{\partial \Omega}+\mathrm{m}_{1}^{\Omega} .
$$

As explained above, the set $\mathrm{U}_{1}^{\bar{\Omega}}$ is the set of the generalized saddle points of $f$. If $\mathrm{U}_{1}^{\partial \Omega}$ is not empty, its elements are denoted by:

$$
\mathrm{U}_{1}^{\partial \Omega}=\left\{z_{1}, \ldots, z_{\mathrm{m}_{1}^{\partial \Omega}}\right\} \subset \partial \Omega
$$

and if $\mathrm{U}_{1}^{\Omega}$ is not empty, its elements are labeled as follows:

$$
\mathrm{U}_{1}^{\Omega}=\left\{z_{\mathrm{m}_{1}^{\partial \Omega}+1}, \ldots, z_{\mathrm{m}_{1}^{\bar{\Omega}}}\right\} \subset \Omega
$$

Thus, one has: $\mathrm{U}_{1}^{\bar{\Omega}}=\left\{z_{1}, \ldots, z_{\mathrm{m}_{1}^{\partial \Omega}}, z_{\mathrm{m}_{1}^{\partial \Omega}+1}, \ldots, z_{\mathrm{m}_{1}^{\bar{\Omega}}}\right\}$. Moreover, we assume that the elements of $\mathrm{U}_{1}^{\partial \Omega}$ are ordered such that:

$$
\left\{z_{1}, \ldots, z_{\mathrm{k}_{1}^{\partial \Omega}}\right\}=\bigcup_{1}^{\partial \Omega} \cap \underset{\partial \Omega}{\arg \min } f .
$$

Notice that $\mathrm{k}_{1}^{\partial \Omega} \in\left\{0, \ldots, \mathrm{m}_{1}^{\partial \Omega}\right\}$. 

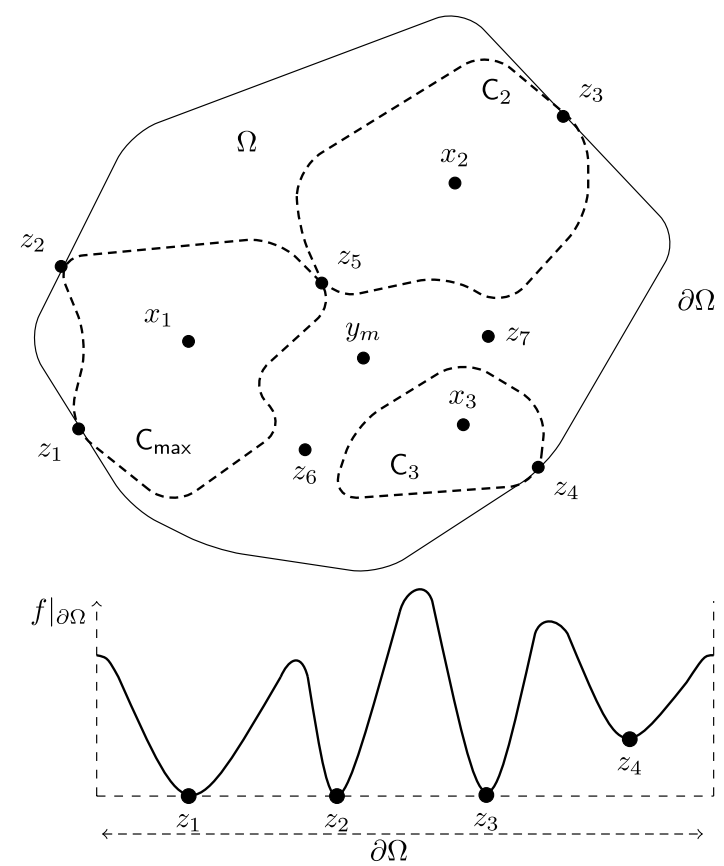

Fig. 2. Schematic representation of $\mathcal{C}$ (see (14)) and $\left.f\right|_{\partial \Omega}$ when the assumptions (A0), (A1), (A2) and (A3) are satisfied. In this representation, $x_{1} \in \Omega$ is the global minimum of $f$ in $\bar{\Omega}$ and the other local minima of $f$ in $\Omega$ are $x_{2}$ and $x_{3}\left(\right.$ thus $\mathrm{U}_{0}^{\Omega}=\left\{x_{1}, x_{2}, x_{3}\right\}$ and $\mathrm{m}_{0}^{\Omega}=3$ ). Moreover, $\min _{\partial \Omega} f=f\left(z_{1}\right)=f\left(z_{2}\right)=f\left(z_{3}\right)=\mathrm{H}_{f}\left(x_{1}\right)=\mathrm{H}_{f}\left(x_{2}\right)<\mathrm{H}_{f}\left(x_{3}\right)=f\left(z_{4}\right),\left\{f<\mathrm{H}_{f}\left(x_{1}\right)\right\}$ has two connected components: $\mathrm{C}_{\max }$ (see (A1)) which contains $x_{1}$ and $\mathrm{C}_{2}$ which contains $x_{2}$. Thus, one has $\mathcal{C}=\left\{\mathrm{C}_{\max }, \mathrm{C}_{2}, \mathrm{C}_{3}\right\}$. In addition, $\mathrm{U}_{1}^{\partial \Omega}=\left\{z_{1}, z_{2}, z_{3}, z_{4}\right\}\left(\mathrm{m}_{1}^{\partial \Omega}=4\right),\left\{z_{1}, z_{2}, z_{3}\right\}=\arg \min _{\partial \Omega} f\left(\mathrm{k}_{1}^{\partial \Omega}=3\right.$ and $\left.\mathrm{k}_{1}^{\partial \mathrm{C}_{\max }}=2\right), \mathrm{U}_{1}^{\Omega}=\left\{z_{5}, z_{6}, z_{7}\right\}$ where $\left\{z_{5}\right\}=\overline{\mathrm{C}_{\max }} \cap \overline{\mathrm{C}_{2}}$ $\left(\mathrm{m}_{1}^{\Omega}=3\right.$ and (A4) is not satisfied) and $\min \left(f\left(z_{6}\right), f\left(z_{7}\right)\right)>f\left(z_{4}\right), \partial \mathrm{C}_{\max } \cap \partial \Omega=\left\{z_{1}, z_{2}\right\}\left(\mathrm{k}_{1}^{\partial \mathrm{C}_{\max }}=2\right)$. Finally, one has $\mathrm{m}_{1}^{\bar{\Omega}}=7$. The point $y_{m} \in \Omega$ is a local maximum of $f$ with $f\left(y_{m}\right)>f\left(z_{i}\right)$ for all $i \in\{1, \ldots, 7\}$.

Let us assume that (A1), (A2), and (A3) are satisfied. Let us recall that $\mathrm{C}_{\max }$ is defined by (A1). Moreover, in this case, one has $\mathrm{k}_{1}^{\partial \Omega} \geq 1$ and

$$
\partial \mathrm{C}_{\max } \cap \partial \Omega \subset\left\{z_{1}, \ldots, z_{\mathrm{k}_{1}^{\partial \Omega}}\right\} .
$$

Indeed, by assumption $\partial \mathrm{C}_{\max } \cap \partial \Omega \subset\left\{f=\min _{\partial \Omega} f\right\}$ (see (A3)) and there is no local minima of $f$ in $\bar{\Omega}$ on $\partial \mathrm{C}_{\max }$ ( since $\mathrm{C}_{\max }$ is a connected component of a sublevel set of $f$ ). We assume lastly that the set $\left\{z_{1}, \ldots, z_{\mathrm{k}_{1}^{\partial \Omega}}\right\}$ is ordered such that:

$$
\left\{z_{1}, \ldots, z_{\mathrm{k}_{1}^{\partial C_{\max }}}\right\}=\left\{z_{1}, \ldots, z_{\mathrm{k}_{1}^{\partial \Omega}}\right\} \cap \partial \mathrm{C}_{\max } .
$$

Notice that $\mathrm{k}_{1}^{\partial \mathrm{C}_{\max }} \in\left\{0, \ldots, \mathrm{k}_{1}^{\partial \Omega}\right\}$. We provide an example in Fig. 2 to illustrate the notations introduced in this section.

As introduced in [23, Section 5.2], $\mathrm{U}_{0}^{\Omega}$ is the set of generalized critical points of $f$ of index 0 , associated with eigenforms of the Witten Laplacian $\Delta_{f, h}^{D,(0)}$ and $\bigcup_{1}^{\bar{\Omega}}$ is the set of generalized critical points of $f$ of index 1 , associated with eigenforms of the Witten Laplacian $\Delta_{f, h}^{D,(1)}$, see Section 3.1.2 for more details.

\subsubsection{Main results on the exit point distribution}

The main result of this work is the following.

Theorem 1. Let us assume that (A0), (A1), (A2), and (A3) are satisfied. Recall that $\nu_{h}$ is the quasi-stationary distribution of the process (1) in $\Omega$ (see Definition 2). Let $F \in L^{\infty}(\partial \Omega, \mathbb{R})$ and $\left(\Sigma_{i}\right)_{i \in\left\{1, \ldots, \mathrm{k}_{1}^{\text {a }}\right\}}$ be a family of disjoint open subsets of $\partial \Omega$ such that for all $i \in\left\{1, \ldots, \mathrm{k}_{1}^{\partial \Omega}\right\}, z_{i} \in \Sigma_{i}$, where we recall that $\left\{z_{1}, \ldots, z_{\mathrm{k}_{1}^{\partial \Omega}}\right\}=$ $\mathrm{U}_{1}^{\partial \Omega} \cap \arg \min _{\partial \Omega} f($ see (22)). Then: 
1. There exists $c>0$ such that in the limit $h \rightarrow 0$ :

$$
\mathbb{E}_{\nu_{h}}\left[F\left(X_{\tau_{\Omega}}\right)\right]=\sum_{i=1}^{\mathrm{k}_{1}^{\partial \Omega}} \mathbb{E}_{\nu_{h}}\left[\mathbf{1}_{\Sigma_{i}} F\left(X_{\tau_{\Omega}}\right)\right]+O\left(e^{-\frac{c}{h}}\right)
$$

and

$$
\sum_{i=\mathrm{k}_{1}^{\partial \mathrm{C}_{\max }}+1}^{\mathrm{k}_{1}^{\partial \Omega}} \mathbb{E}_{\nu_{h}}\left[\mathbf{1}_{\Sigma_{i}} F\left(X_{\tau_{\Omega}}\right)\right]=O\left(h^{\frac{1}{4}}\right)
$$

where we recall that $\left\{z_{1}, \ldots, z_{\mathrm{k}_{1}^{\partial c_{\max }}}\right\}=\partial \mathrm{C}_{\max } \cap \partial \Omega$ (see (23)).

2. When for some $i \in\left\{1, \ldots, \mathrm{k}_{1}^{\partial \mathcal{C}_{\max }}\right\}$ the function $F$ is $C^{\infty}$ in a neighborhood of $z_{i}$, one has when $h \rightarrow 0$ :

$$
\mathbb{E}_{\nu_{h}}\left[\mathbf{1}_{\Sigma_{i}} F\left(X_{\tau_{\Omega}}\right)\right]=F\left(z_{i}\right) a_{i}+O\left(h^{\frac{1}{4}}\right)
$$

where

$$
a_{i}=\frac{\partial_{n} f\left(z_{i}\right)}{\sqrt{\left.\operatorname{det} \operatorname{Hess} f\right|_{\partial \Omega}\left(z_{i}\right)}}\left(\sum_{j=1}^{k_{1}^{\partial c_{\max }}} \frac{\partial_{n} f\left(z_{j}\right)}{\sqrt{\left.\operatorname{det} \operatorname{Hess} f\right|_{\partial \Omega}\left(z_{j}\right)}}\right)^{-1} .
$$

3. When (A4) is satisfied, the remainder term $O\left(h^{\frac{1}{4}}\right)$ in (25) is of the order $O\left(e^{-\frac{c}{h}}\right)$ for some $c>0$ and the remainder term $O\left(h^{\frac{1}{4}}\right)$ in (26) is of the order $O(h)$ and admits a full asymptotic expansion in $h$ (as defined in Remark 7 below).

Remark 7. Let us recall that for $\alpha>0,(r(h))_{h>0}$ admits a full asymptotic expansion in $h^{\alpha}$ if there exists a sequence $\left(a_{k}\right)_{k \geq 0} \in \mathbb{R}^{\mathbb{N}}$ such that for any $N \in \mathbb{N}$, it holds in the limit $h \rightarrow 0$ :

$$
r(h)=\sum_{k=0}^{N} a_{k} h^{\alpha k}+O\left(h^{\alpha(N+1)}\right) .
$$

Theorem 1 implies that in the limit $h \rightarrow 0$, when $X_{0} \sim \nu_{h}$, the law of $X_{\tau_{\Omega}}$ admits a limit in distribution and concentrates on the set $\left\{z_{1}, \ldots, z_{\mathrm{k}_{1}} \mathrm{C}_{\max }\right\}=\partial \Omega \cap \partial \mathrm{C}_{\max }$ with explicit formulas for the probabilities to exit through each of the $z_{i}$ 's.

As a simple corollary, notice that when the function $F$ belongs to $C^{\infty}(\partial \Omega, \mathbb{R})$, one has in the limit $h \rightarrow 0$ :

$$
\mathbb{E}_{\nu_{h}}\left[F\left(X_{\tau_{\Omega}}\right)\right]=\sum_{i=1}^{\mathrm{k}_{1}^{\partial c_{\max }}} a_{i} F\left(z_{i}\right)+O\left(h^{\frac{1}{4}}\right)=\frac{\sum_{i=1}^{\mathrm{k}_{1}^{\partial c_{\max }}} \int_{\Sigma_{i}} F \partial_{n} f e^{-\frac{2}{h} f}}{\sum_{i=1}^{\mathrm{k}_{1}^{\partial C_{\max }}} \int_{\Sigma_{i}} \partial_{n} f e^{-\frac{2}{h} f}}+o_{h}(1),
$$

where the order in $h$ of the remainder term $o_{h}(1)$ depends on the support of $F$ and on whether or not the assumption (A4) is satisfied.

Another consequence of Theorem 1 is the following. The probability to exit through a global minimum $z$ of $\left.f\right|_{\partial \Omega}$ which satisfies $\partial_{n} f(z)<0$ is exponentially small in the limit $h \rightarrow 0$ (see (24)) and when assuming (A4), the probability to exit through the points $z_{\mathrm{k}_{1}^{c_{\max }}+1}, \ldots, z_{\mathrm{k}_{1}^{\partial \Omega}}$ is also exponentially small even though all these points belong to $\arg \min _{\partial \Omega} f$. 
Remark 8. In [18], we show that the results of Theorem 1 still hold when $X_{0}=x \in \mathrm{C}_{\max }$. Moreover, we also prove that when $X_{0}=x \in \mathrm{C}$, for $\mathrm{C} \in \mathcal{C}$ such that $\partial \mathrm{C} \cap \partial \Omega \neq \emptyset$, the law of $X_{\tau_{\Omega}}$ concentrates on $\partial \mathrm{C} \cap \partial \Omega$ when $h \rightarrow 0$, with explicit exit probabilities. We also refer to the preprint [24] which concatenates the results of this manuscript and of [18], and to [25] which presents a simplified version of the results of these works.

The proof of Theorem 1 relies on a crucial result on the concentration of the quasi-stationary distribution on neighborhoods of the global minima of $f$ in $C_{\max }$.

Proposition 9. Assume that (A0) and (A1) are satisfied. Furthermore, let us assume that

$$
\min _{\overline{C_{\max }}} f=\min _{\bar{\Omega}} f
$$

where we recall that $\mathrm{C}_{\max }$ is introduced in (A1). Let $\mathrm{O}$ be an open subset of $\Omega$. Then, if $\mathrm{O} \cap \arg \min _{\mathrm{C}_{\max }} f \neq \emptyset$, one has in the limit $h \rightarrow 0$ :

$$
\nu_{h}(\mathrm{O})=\frac{\sum_{x \in \mathrm{O} \operatorname{\mathrm {arg}} \min _{\mathrm{C}_{\max }} f}(\operatorname{det} \operatorname{Hess} f(x))^{-\frac{1}{2}}}{\sum_{x \in \arg \min _{\mathrm{C}_{\max }} f}(\operatorname{det} \operatorname{Hess} f(x))^{-\frac{1}{2}}}(1+O(h)) .
$$

When $\overline{\mathrm{O}} \cap \arg \min _{\mathrm{C}_{\text {max }}} f=\emptyset$, there exists $c>0$ such that when $h \rightarrow 0$ :

$$
\nu_{h}(\mathrm{O})=O\left(e^{-\frac{c}{h}}\right)
$$

Proposition 9 is a direct consequence of (11) and Proposition 58 below. Notice that $\min _{\overline{\mathrm{C}_{\max }}} f=\min _{\bar{\Omega}} f$ is satisfied when (A1), (A2), and (A3) hold, see Lemma 22.

\subsubsection{Intermediate results on the spectrum of $-L_{f, h}^{D,(0)}$}

Let us recall that from (12), one has:

$$
\mathbb{E}_{\nu_{h}}\left[F\left(X_{\tau_{\Omega}}\right)\right]=-\frac{h}{2 \lambda_{h}} \frac{\int_{\Omega \Omega} F \partial_{n} u_{h} e^{-\frac{2}{h} f}}{\int_{\Omega} u_{h} e^{-\frac{2}{h} f}} .
$$

Therefore, to obtain the asymptotic estimates on $\mathbb{E}_{\nu_{h}}\left[F\left(X_{\tau_{\Omega}}\right)\right]$ stated in Theorem 1 when $h \rightarrow 0$, it is sufficient to study the asymptotic behavior of the quantities $\lambda_{h}, \int_{\Omega} u_{h} e^{-\frac{2}{h} f}$ and $\partial_{n} u_{h}$. Let us point to the results which will be proven below on these quantities, and which may have their own interest:

1. In Theorem 4, one gives for $h \rightarrow 0$ small enough, a lower and an upper bound for all the $\mathrm{m}_{0}^{\Omega}$ small eigenvalues of $-L_{f, h}^{D,(0)}$ when (A0) is satisfied.

2. In Theorems 2 and 3, one gives a sharp asymptotic equivalent in the limit $h \rightarrow 0$ of the smallest eigenvalue $\lambda_{h}$ of $-L_{f, h}^{D,(0)}$ when (A0) and (A1) are satisfied.

3. In Proposition 58, when (A0), (A1) and $\min _{\overline{C_{\max }}} f=\min _{\bar{\Omega}} f$ hold, one shows that $u_{h} e^{-\frac{2}{h} f}$ concentrates in the $L^{1}(\Omega)$-norm on the global minima of $f$ in $C_{\max }$ in the limit $h \rightarrow 0$.

4. In Theorem 5 , one studies the concentration in the limit $h \rightarrow 0$ of the normal derivative of the principal eigenfunction $u_{h}$ of $-L_{f, h}^{D,(0)}$ on $\partial \Omega$ when (A0), (A1), (A2), and (A3) are satisfied. In particular, sharp asymptotic equivalents of $\partial_{n} u_{h}$ in neighborhoods of $\partial \mathrm{C}_{\max } \cap \partial \Omega$ in $\partial \Omega$ are obtained. 


\subsection{Discussion of the hypotheses}

In this section, we discuss the necessity of the assumptions (A1), (A2), (A3) and (A4) to obtain the results stated in Theorem 1.

On the assumption (A1). To study the concentration of the law of $X_{\tau_{\Omega}}$ when $h \rightarrow 0$ when $X_{0} \sim \nu_{h}$, one needs in particular to have access to the repartition of $\nu_{h}$ in neighborhoods of the local minima of $f$ in $\Omega$ when $h \rightarrow 0$. When (A1) is not satisfied, the analysis of the repartition of $\nu_{h}$ is tricky. When (A1) is not satisfied, one has from Theorem 4 below (see Section 4.2.2), $\lim _{h \rightarrow 0} h \ln \lambda_{h}=\lim _{h \rightarrow 0} h \ln \lambda_{2, h}$, where $\lambda_{2, h}$ is the second smallest eigenvalue of $-L_{f, h}^{D,(0)}$. The first two eigenvalues thus have the same exponential scaling in $h$. As a consequence, it is difficult to measure the quality of the approximation of $u_{h}$ by an ansatz projected on $\operatorname{Span}\left(u_{h}\right)$, since the error is related to the ratio of $\lambda_{h}$ over $\lambda_{2, h}$ (see Lemma 25). For example, when (A1) is not satisfied, it is difficult to predict in which well $\nu_{h}$ concentrates, as explained in [26].

On the assumptions (A2) and (A3). When (A1) is satisfied but not (A2), or when (A1), (A2) are satisfied but not (A3), it is possible to exhibit functions $f:\left[z_{1}, z_{2}\right] \rightarrow \mathbb{R}\left(\right.$ with $\left.f\left(z_{1}\right)<f\left(z_{2}\right)\right)$ such that $\mathbb{P}_{\nu_{h}}\left[X_{\tau_{\left(z_{1}, z_{2}\right)}}=\right.$ $\left.z_{2}\right]=1+O\left(e^{-\frac{c}{h}}\right)$ for some positive constant $c$ (see [24, Section 1.4.3, Section 1.4.4] for details). In those cases, the process (1) thus leaves $\Omega=\left(z_{1}, z_{2}\right)$ through the point $z_{2}$ which is not a global minimum of $\left.f\right|_{\partial \Omega}$.

On the assumption (A4). In [24, Section 1.4.5], we give a one-dimensional example to show that when (A4) is not satisfied, the remainder term $O\left(h^{\frac{1}{4}}\right)$ in $(25)$ is not of the order $O\left(e^{-\frac{c}{h}}\right)$ for some $c>0$, but actually of the order $O(\sqrt{h})$. This can be generalized to higher-dimensional settings, see for example [27, Proposition C.40, item 3]. We thus expect that the remainder terms $O\left(h^{\frac{1}{4}}\right)$ in (25) and (26) are actually of the order $O(\sqrt{h})$, but proving this fact would require some substantially finer analysis.

\subsection{Organization of the paper and outline of the proof}

The aim of this section is to give an overview of the strategy of the proof of Theorem 1. From (12) and in order to obtain an asymptotic estimate of $\mathbb{E}_{\nu_{h}}\left[F\left(X_{\tau_{\Omega}}\right)\right]$, we study the asymptotic behavior when $h \rightarrow 0$ of the quantities $\lambda_{h}, \int_{\Omega} u_{h} e^{-\frac{2}{h} f}$ and $\partial_{h} u_{h}$, where $\lambda_{h}$ is defined by (8) and $u_{h}$ by (10).

To study $\lambda_{h}$ and $\partial_{n} u_{h}$, the first key point is to notice that $\nabla u_{h}$ is a solution to an eigenvalue problem. Indeed, by differentiating the eigenvalue problem (10) satisfied by $u_{h}$, one gets:

$$
\left\{\begin{aligned}
-L_{f, h}^{(1)} \nabla u_{h} & =\lambda_{h} \nabla u_{h} \text { on } \Omega, \\
\nabla_{T} u_{h} & =0 \text { on } \partial \Omega, \\
\left(\frac{h}{2} \operatorname{div}-\nabla f \cdot\right) \nabla u_{h} & =0 \text { on } \partial \Omega,
\end{aligned}\right.
$$

where

$$
L_{f, h}^{(1)}=\frac{h}{2} \Delta-\nabla f \cdot \nabla-\operatorname{Hess} f
$$

is an operator acting on 1-forms (namely on vector fields). Therefore, the vector field $\nabla u_{h}$ is an eigen-1-form of the operator $-L_{f, h}^{D,(1)}$ which is the operator $-L_{f, h}^{(1)}$ with tangential Dirichlet boundary conditions (see (28)), associated with the eigenvalue $\lambda_{h}$.

The second key point (see for example [23]) is that, when (A0) holds, $-L_{f, h}^{D,(0)}$ admits exactly $\mathrm{m}_{0}^{\Omega}$ eigenvalues smaller than $\frac{\sqrt{h}}{2}$ (where $\mathrm{m}_{0}^{\Omega}$ is the number of local minima of $f$ in $\Omega$, see Section 1.3.2) and that $-L_{f, h}^{D,(1)}$ admits exactly $\mathrm{m}_{1}^{\bar{\Omega}}$ eigenvalues smaller than $\frac{\sqrt{h}}{2}$ (where $\mathrm{m}_{1}^{\bar{\Omega}}$ is the number of generalized saddle points of $f$ in $\bar{\Omega}$, see Section 1.3.2). Actually, all these small eigenvalues are exponentially small in the regime $h \rightarrow 0$ (namely they are bounded from above by $e^{-\frac{c}{h}}$ for some $c>0$ ), the other eigenvalues being bounded from 
below by a constant in this regime. This implies in particular that $\lambda_{h}$ is an exponentially small eigenvalue of $-L_{f, h}^{D,(1)}$. Let us denote by $\pi_{h}^{(0)}$ (resp. $\pi_{h}^{(1)}$ ) the orthogonal projector onto the vector space spanned by the eigenfunctions (resp. eigen-1-forms) associated with the $\mathrm{m}_{0}^{\Omega}$ (resp. $\mathrm{m}_{1}^{\bar{\Omega}}$ ) smallest eigenvalue of $-L_{f, h}^{D,(0)}$ (resp. of $\left.-L_{f, h}^{D,(1)}\right)$.

To obtain an asymptotic estimate on $\lambda_{h}$ when $h \rightarrow 0$, the strategy consists in studying the singular values of the (finite-dimensional) operator $\nabla$ acting from $\operatorname{Ran} \pi_{h}^{(0)}$ to $\operatorname{Ran} \pi_{h}^{(1)}$, both spaces being equipped with the scalar product of $L_{w}^{2}(\Omega)$. Indeed, from Proposition 4, the squares of the singular values of this matrix are the smallest eigenvalues of $-\frac{2}{h} L_{f, h}^{D,(0)}$. To this end, one constructs an appropriate basis (with so called quasi-modes) of $\operatorname{Ran} \pi_{h}^{(0)}$ and $\operatorname{Ran} \pi_{h}^{(1)}$. Besides, from (28), $\nabla u_{h} \in \operatorname{Ran} \pi_{h}^{(1)}$ and thus, to study the asymptotic behavior of $\partial_{n} u_{h}$ on $\partial \Omega$ when $h \rightarrow 0$, one decomposes $\nabla u_{h}$ along a basis of $\operatorname{Ran} \pi_{h}^{(1)}$.

In terms of mathematical tools, the proofs heavily rely on constructions made in $[28,23]$. The main novelty is to combine those techniques to get precise estimates of $\partial_{n} u_{h}$ : this requires to go beyond the standard semiclassical estimates which focus on the eigenvalues. The crucial steps in the proofs are: (i) Proposition 47 which gives the interaction terms between the quasi-modes for $L_{f, h}^{D,(0)}$ and for $L_{f, h}^{D,(1)}$, (ii) Lemma 50 which uses a matrix representation of the operator $\nabla: \operatorname{Ran} \pi_{h}^{(0)} \mapsto \operatorname{Ran} \pi_{h}^{(1)}$, thanks to an appropriate labeling of the connected components attached to the local minima of $f$ and (iii) Lemma 63 which explains how to get $H_{w}^{1}$-estimates of the error between $u_{h}$ and its approximation by quasi-modes.

The paper is organized as follows. In Section 2, one constructs two maps $\mathbf{j}$ and $\widetilde{\mathbf{j}}$ which will be extensively used in Section 3. These maps are useful in order to understand the different timescales of the process (1) in $\Omega$. Section 3 is dedicated to the construction of quasi-modes for $-L_{f, h}^{D,(0)}$ and $-L_{f, h}^{D,(1)}$. In Section 4 , we study the asymptotic behaviors of the smallest eigenvalues of $-L_{f, h}^{D,(0)}$ (see Theorem 4 ) and we give an asymptotic estimate of $\lambda_{h}$ when $h \rightarrow 0$, see Theorem 2. In Section 5, we give asymptotic estimates for $\int_{\Omega} u_{h} e^{-\frac{2}{h} f}$ and for $\partial_{n} u_{h}$ on $\partial \Omega$ when $h \rightarrow 0$ (see Proposition 58 and Theorem 5), and we finally conclude the proof of Theorem 1. For the ease of the reader, a list of the main notation used in this work is provided at the end.

\section{Coupling local minima of $f$ with saddle points of $f$}

This section is dedicated to the construction of two maps: the map $\mathbf{j}$ which associates each local minimum of $f$ with an ensemble of saddle points of $f$ and the map $\widetilde{\mathbf{j}}$ which associates each local minimum of $f$ with a connected component of a sublevel set of $f$. These maps are useful to define the quasi-modes in Section 3.

This section is organized as follows. In Section 2.1, one introduces a set of connected components which play a crucial role in our analysis. The constructions of the maps $\mathbf{j}$ and $\widetilde{\mathbf{j}}$ require two preliminary results (Propositions 15 and 18) which are introduced in Section 2.2. Then, the maps $\mathbf{j}$ and $\widetilde{\mathbf{j}}$ are defined in Section 2.3. Finally the assumptions (A1)-(A4) are reformulated in terms of the map $\mathbf{j}$ in Section 2.4.

\subsection{Connected components associated with the elements of $\mathrm{U}_{0}^{\Omega}$}

The aim of this section is to give an equivalent definition of the elements in $\mathcal{C}=\left\{\mathrm{C}(x), x \in \mathrm{U}_{0}^{\Omega}\right\}$ (see (14) and (16)) which will be easier to handle in the following. For that purpose, let us introduce the following definitions.

Definition 10. Let us assume that (A0) holds. For all $x \in \mathrm{U}_{0}^{\Omega}$ and $\lambda>f(x)$, one defines

$$
\mathrm{C}(\lambda, x) \text { as the connected component of }\{f<\lambda\} \text { in } \bar{\Omega} \text { containing } x
$$




$$
\mathrm{C}^{+}(\lambda, x) \text { as the connected component of }\{f \leq \lambda\} \text { in } \bar{\Omega} \text { containing } x \text {. }
$$

Moreover, for all $x \in \mathrm{U}_{0}^{\Omega}$, one defines

$$
\lambda(x):=\sup \{\lambda>f(x) \text { s.t. } \mathrm{C}(\lambda, x) \cap \partial \Omega=\emptyset\} \quad \text { and } \quad \mathrm{C}(x):=\mathrm{C}(\lambda(x), x) .
$$

A direct consequence of Lemma 11 below is that for all $x \in \mathrm{U}_{0}^{\Omega}, \mathrm{C}(x)$ defined in (32) coincides with $\mathrm{C}(x)$ introduced in (15). Notice that under (A0), for all $x \in \mathrm{U}_{0}^{\Omega} \subset \Omega, \lambda(x)$ is well defined. Indeed, for all $x \in \mathrm{U}_{0}^{\Omega}$, $\{\lambda>f(x)$ s.t. $\mathrm{C}(\lambda, x) \cap \partial \Omega=\emptyset\}$ is bounded by $\sup _{\bar{\Omega}} f+1$ and nonempty because for $\beta>0$ small enough $\mathrm{C}(f(x)+\beta, x)$ is included in $\Omega$ (since $x \in \Omega$ and $f$ is Morse). One has the following result which permits to give another definition of $\mathbf{H}_{f}$ (compare with (13)) which will be easier to handle in the sequel.

Lemma 11. Let us assume that (A0) holds. Then, for all $x \in \mathrm{U}_{0}^{\Omega}$

$$
\mathrm{H}_{f}(x)=\lambda(x),
$$

where $\mathrm{H}_{f}(x)$ is defined by (13) and $\lambda(x)$ is defined by (32).

The proof of Lemma 11 is made in [24, Lemma 15].

Definition 12. Let us assume that (A0) holds. The integer $\mathrm{N}_{1}$ is defined by:

$$
\mathrm{N}_{1}:=\operatorname{Card}(\mathcal{C})=\operatorname{Card}\left(\left\{\mathrm{C}(x), x \in \mathrm{U}_{0}^{\Omega}\right\}\right) \in\left\{1, \ldots, \mathrm{m}_{0}^{\Omega}\right\}
$$

where we recall that $\mathrm{m}_{0}^{\Omega}=\mathrm{Card}\left(\mathrm{U}_{0}^{\Omega}\right)$ (see (16)), $\mathrm{C}(x)$ is defined by (32) and $\mathcal{C}=\left\{\mathrm{C}(x), x \in \mathrm{U}_{0}^{\Omega}\right\}$ (see (14)). The elements of $\mathcal{C}$ are denoted by $\mathrm{C}_{1}, \ldots, \mathrm{C}_{\mathrm{N}_{1}}$. Finally, for all $\ell \in\left\{1, \ldots, \mathrm{N}_{1}\right\}, \mathrm{C}_{k}$ is also denoted by

$$
\mathrm{E}_{1, \ell}:=\mathrm{C}_{\ell}
$$

For example, on Fig. 1 , one has $\mathrm{m}_{0}^{\Omega}=4$ and $\mathrm{N}_{1}=3$. The notation (35) will be useful when constructing the maps $\mathbf{j}$ and $\widetilde{\mathbf{j}}$ in Section 2.3 below.

\subsection{Topological results and separating saddle points}

The constructions of the maps $\mathbf{j}$ and $\widetilde{\mathbf{j}}$ made in Section 2.3 are based on the notions of separating saddle points and of critical components as introduced in [28, Section 4.1] for a case without boundary. Let us define and slightly adapt these two notions to our setting. To this end, let us first recall that according to [23, Section 5.2], for any non critical point $z \in \Omega$, for $r>0$ small enough

$$
\{f<f(z)\} \cap B(z, r) \text { is connected, }
$$

and for any critical point $z \in \Omega$ of index $p$ of the Morse function $f$, for $r>0$ small enough, one has the three possible cases:

$$
\left\{\begin{array}{l}
\text { either } p=0(z \text { is a local minimum of } f) \text { and }\{f<f(z)\} \cap B(z, r)=\emptyset, \\
\text { or } p=1 \text { and }\{f<f(z)\} \cap B(z, r) \text { has exactly two connected components, } \\
\text { or } p \geq 2 \text { and }\{f<f(z)\} \cap B(z, r) \text { is connected, }
\end{array}\right.
$$

where $B(z, r):=\{x \in \bar{\Omega}$ s.t. $|x-z|<r\}$. The separating saddle points of $f$ and the critical components of $f$ are defined as follows (see Fig. 3 for an illustration): 


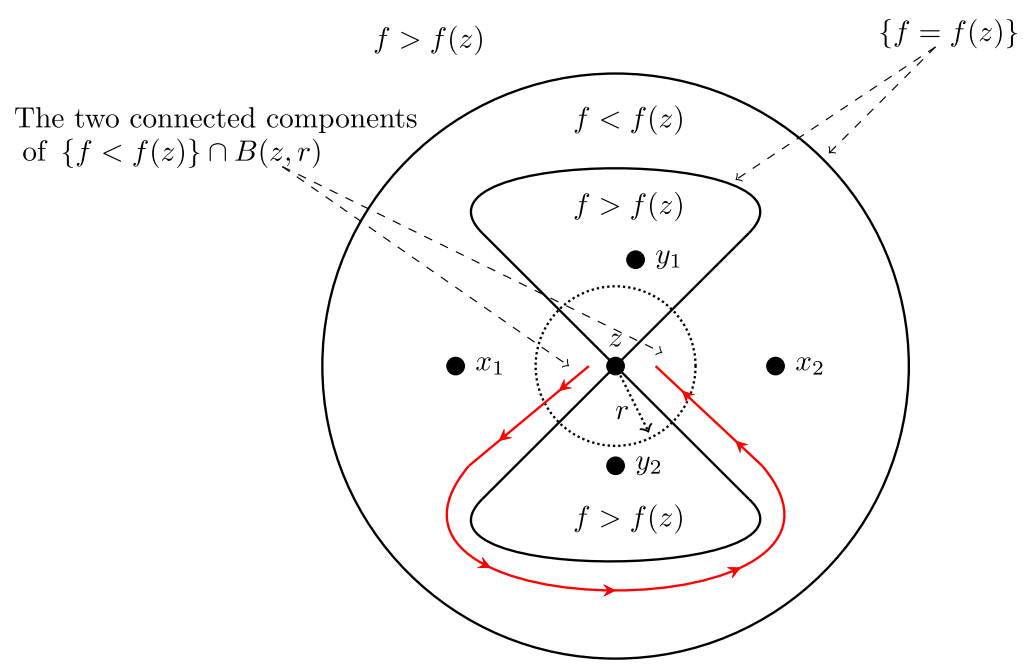

Fig. 3. An example in dimension 2 of a saddle point which is not separating. The points $x_{1}$ and $x_{2}$ are two local minima of $f$, and the points $y_{1}$ and $y_{2}$ are two local maxima of $f$. The two connected components of $\{f<f(z)\} \cap B(z, r)$ are contained in the same connected components of $\{f<f(z)\}$ (see the arrowed path on the figure).

Definition 13. Assume (A0). Let $\mathcal{C}=\left\{\mathrm{C}_{1}, \ldots, \mathrm{C}_{\mathrm{N}_{1}}\right\}$ be the set of connected sets as introduced in Definition 12.

1. A point $z \in \mathrm{U}_{1}^{\bar{\Omega}}$ is a separating saddle point if either $z \in \mathrm{U}_{1}^{\partial \Omega} \cap \cup_{i=1}^{N_{1}} \partial \mathrm{C}_{i}$ or $z \in \mathrm{U}_{1}^{\Omega} \cap \cup_{i=1}^{N_{1}} \overline{C_{i}}$ and for $r>0$ small enough, the two connected components of $\{f<f(z)\} \cap B(z, r)$ are contained in different connected components of $\{f<f(z)\}$. Notice that in the former case $z \in \partial \Omega$ while in the latter case $z \in \Omega$. The set of separating saddle points is denoted by $\mathrm{U}_{1}^{\text {ssp }}$.

2. For any $\sigma \in \mathbb{R}$, a connected component $\mathrm{E}$ of the sublevel set $\{f<\sigma\}$ in $\bar{\Omega}$ is called a critical connected component if $\partial \mathrm{E} \cap \mathrm{U}_{1}^{\text {ssp }} \neq \emptyset$. The family of critical connected components is denoted by $\mathcal{C}_{\text {crit }}$.

Remark 14. It is natural to define generally a separating saddle point of a Morse function $f$ as follows: $z$ is a separating saddle point if for any sufficiently small connected neighborhood $\mathcal{V}_{z}$ of $z, \mathcal{V}_{z} \cap\{f<f(z)\}$ has two connected components included in two different connected components of $\{f<f(z)\}$. Our definition of separating saddle point is equivalent to this general definition when the function $f$ is extended by $-\infty$ outside $\bar{\Omega}$. In particular, with this extended definition of $f$, there cannot be a separating saddle point outside $\cup_{i=1}^{\mathrm{N}_{1}} \overline{\mathrm{C}_{i}}$. We refer to [24, Remark 19] for more details.

In the rest of this section, we give a series of results on $\left\{C_{1}, \ldots, C_{N_{1}}\right\}$ which will be used throughout this paper. These results are rather intuitive and, for the sake of conciseness, we refer to [24] for detailed proofs.

Proposition 15. Let us assume that $(\mathrm{A} 0)$ holds. Let $\mathcal{C}=\left\{\mathrm{C}_{1}, \ldots, \mathrm{C}_{\mathrm{N}_{1}}\right\}$ be the set of connected sets introduced in Definition 12 and let $(k, \ell) \in\left\{1, \ldots, \mathrm{N}_{1}\right\}^{2}$ with $k \neq \ell$. Then,

$$
\mathrm{C}_{k} \text { is an open subset of } \Omega \text { and } \mathrm{C}_{k} \cap \mathrm{C}_{\ell}=\emptyset \text {. }
$$

In addition, one has

$$
\partial \mathrm{C}_{k} \cap \partial \Omega \subset \mathrm{U}_{1}^{\text {ssp }} \cap \partial \Omega \text { and } \partial \mathrm{C}_{k} \cap \partial \mathrm{C}_{\ell} \subset \mathrm{U}_{1}^{\text {ssp }} \cap \Omega,
$$

where the set $\mathrm{U}_{1}^{\mathrm{ssp}}$ is introduced in item 1 in Definition 13. Finally, $\partial \mathrm{C}_{k} \cap \mathrm{U}_{1}^{\text {ssp }} \neq \emptyset$.

The proof of Proposition 15 is made in [24, Proposition 20] and is based on the following technical lemma (see [24, Lemma 17]). 
Lemma 16. Let us assume that the function $f: \bar{\Omega} \rightarrow \mathbb{R}$ is a $C^{\infty}$ function. Let $x \in \mathrm{U}_{0}^{\Omega}$. For all $\mu>f(x)$, it holds $\mathrm{C}(\mu, x)=\bigcup_{\lambda<\mu} \mathrm{C}(\lambda, x)$ and $\mathrm{C}^{+}(\mu, x)=\bigcap_{\lambda>\mu} \mathrm{C}^{+}(\lambda, x)$ where $\mathrm{C}(\mu, x)$ and $\mathrm{C}^{+}(\mu, x)$ are respectively defined in (30) and (31).

The following technical result will be needed in the sequel.

Lemma 17. Let us assume that (A0) is satisfied. Let $\mathcal{C}=\left\{\mathrm{C}_{1}, \ldots, \mathrm{C}_{\mathrm{N}_{1}}\right\}$ be the set of connected sets introduced in Definition 12. Let us consider $\left\{j_{1}, \ldots, j_{k}\right\} \subset\left\{1, \ldots, \mathrm{N}_{1}\right\}$ with $k \in\left\{1, \ldots, \mathrm{N}_{1}\right\}$ and $j_{1}<\ldots<j_{k}$ such that $\cup_{\ell=1}^{k} \overline{\mathrm{C}_{j_{\ell}}}$ is connected and such that for all $q \in\left\{1, \ldots, \mathrm{N}_{1}\right\} \backslash\left\{j_{1}, \ldots, j_{k}\right\}, \overline{\mathrm{C}_{q}} \cap \cup_{\ell=1}^{k} \overline{\mathrm{C}_{j_{\ell}}}=\emptyset$. Then, there exist $z \in \mathrm{U}_{1}^{\mathrm{ssp}}$ and $\ell_{0} \in\{1, \ldots, k\}$ such that

$$
z \in \partial \mathrm{C}_{j_{\ell_{0}}} \backslash\left(\cup_{\ell=1, \ell \neq \ell_{0}}^{k} \partial \mathrm{C}_{j_{\ell}}\right)
$$

The proof of Lemma 17 is made in [24, Lemma 21]. Let us end this section with the following proposition.

Proposition 18. Let us assume that (A0) is satisfied. Let us consider $\mathrm{C}_{q}$ for $q \in\left\{1, \ldots, \mathrm{N}_{1}\right\}$ (see Definition 12). From (30) and (32), there exists $x_{q} \in \mathrm{U}_{0}^{\Omega} \cap \mathrm{C}_{q}$ such that $\mathrm{C}_{q}=\mathrm{C}\left(x_{q}, \lambda\left(x_{q}\right)\right)$. Let $\lambda \in\left(\min _{\overline{C_{q}}} f, \lambda\left(x_{q}\right)\right]$ and $\mathrm{C}$ be a connected component of $\mathrm{C}_{q} \cap\{f<\lambda\}$. Then,

$$
\left(\mathrm{C} \cap \mathrm{U}_{1}^{\text {ssp }} \neq \emptyset\right) \quad \text { iff } \quad \mathrm{C} \cap \mathrm{U}_{0}^{\Omega} \text { contains more than one point. }
$$

Moreover, let us define

$$
\sigma:=\max _{y \in \mathrm{C} \cap U_{1}^{\text {ssp }}} f(y)
$$

with the convention $\sigma=\min _{\overline{\mathrm{C}}} f$ when $\mathrm{C} \cap \mathrm{U}_{1}^{\text {ssp }}=\emptyset$. Then, the following assertions hold.

1. For all $\mu \in(\sigma, \lambda]$, the set $\mathrm{C} \cap\{f<\mu\}$ is a connected component of $\{f<\mu\}$.

2. If $\mathrm{C} \cap \mathrm{U}_{1}^{\text {ssp }} \neq \emptyset$, one has $\mathrm{C} \cap \mathrm{U}_{0}^{\Omega} \subset\{f<\sigma\}$ and the connected components of $\mathrm{C} \cap\{f<\sigma\}$ belong to $\mathcal{C}_{\text {crit }}$.

The proof of Proposition 18 is made in [24, Proposition 22]. It relies on properties of the sublevel sets of a Morse function in $\mathbb{R}^{d}$.

\subsection{Constructions of the maps $\mathbf{j}$ and $\tilde{\mathbf{j}}$}

In this section we construct, under (A0), two maps $\mathbf{j}$ and $\tilde{\mathbf{j}}$, using an association between the local minima $\bigcup_{0}^{\Omega}$ and the (generalized) saddle points $\mathbf{U}_{1}^{\bar{\Omega}}$. Such maps have been introduced in [29-31,28] in the boundaryless case. This has been generalized in [23] to the boundary case (where the authors introduced the notion of generalized saddle points for $\left.\Delta_{f, h}^{D,(0)}\right)$.

Let us recall (see Lemma 23 below), that $L_{f, h}^{D,(0)}$ has exactly $\mathrm{m}_{0}^{\Omega}$ eigenvalues smaller than $\sqrt{h} / 2$ for sufficiently small $h$. Actually, from [32,23], it can be shown that these $\mathrm{m}_{0}^{\Omega}$ eigenvalues are exponentially small. The goal of the map $\mathbf{j}$ is to associate each local minimum $x$ of $f$ with a set of generalized saddle points $\mathbf{j}(x) \subset \mathrm{U}_{1}^{\bar{\Omega}}$ such that $f$ is constant over $\mathbf{j}(x)$ and for sufficiently small $h$,

$$
\exists \lambda \in \sigma\left(-L_{f, h}^{D,(0)}\right), \lim _{h \rightarrow 0} h \ln \lambda=-2(f(\mathbf{j}(x))-f(x)) .
$$

The map $\widetilde{\mathbf{j}}$ associates each local minimum $x$ of $f$ with the connected component of $\{f<f(\mathbf{j}(x))\}$ which contains $x$. To construct the maps $\mathbf{j}$ and $\widetilde{\mathbf{j}}$, the procedure relies on the results of Section 2.2 , following the 
general analysis of the sublevel sets of a Morse function on a manifold without boundary of [28, Section 4.1] which generalizes the procedure described in [31]. The idea is to consider the connected components of $\{f<\lambda\} \cap \bigcup_{1}^{\text {ssp }}$ appearing as $\lambda$ decreases from $\max _{\cup_{k=1}^{N_{1}} \overline{\mathrm{C}_{k}}} f$ to $-\infty$. Each time a new connected component appears in $\cup_{k=1}^{N_{1}} C_{k}$, one picks arbitrarily a global minimum of $f$ in it and then, one associates this local minimum with the separating saddle points on the boundary of this new connected component.

Let us assume that the assumption (A0) holds. The constructions of the maps $\mathbf{j}$ and $\widetilde{\mathbf{j}}$ are made recursively as follows:

1. Initialization $(q=1)$. We consider $\mathrm{E}_{1, \ell}=\mathrm{C}_{\ell}$ for $\ell \in\left\{1, \ldots, \mathrm{N}_{1}\right\}$ (see (35)). For each $\ell \in\left\{1, \ldots, \mathrm{N}_{1}\right\}$, $x_{1, \ell}$ denotes one point in $\arg \min _{\overline{\mathrm{E}}_{1, \ell}} f=\arg \min _{\mathrm{E}_{1, \ell}} f$. Then we define, for all $k \in\left\{1, \ldots, \mathrm{N}_{1}\right\}$,

$$
\sigma_{1, \ell}:=\max _{\overline{\mathrm{E}}_{1, \ell}} f, \tilde{\mathbf{j}}\left(x_{1, \ell}\right):=\mathrm{E}_{1, \ell} \text { and } \mathbf{j}\left(x_{1, \ell}\right):=\partial \mathrm{E}_{1, \ell} \cap \mathrm{U}_{1}^{\text {ssp }}
$$

Notice that according to Proposition 15 and item 2 in Definition 13, it holds

$$
\mathbf{j}\left(x_{1, \ell}\right) \neq \emptyset, \quad \partial \mathrm{E}_{1, \ell} \subset\left\{f=\sigma_{1, \ell}\right\}, \quad \widetilde{\mathbf{j}}\left(x_{1, \ell}\right) \in \mathcal{C}_{\text {crit }} \text { and } \bigcup_{\ell=1}^{\mathrm{N}_{1}} \mathbf{j}\left(x_{1, \ell}\right) \cap \partial \Omega \subset \mathrm{U}_{1}^{\partial \Omega} .
$$

Moreover, one has from Proposition 15 (and more precisely the second inclusion in (39)),

$$
\forall \ell \neq q \in\left\{1, \ldots, \mathrm{N}_{1}\right\}, \partial \mathrm{E}_{1, \ell} \cap \partial \mathrm{E}_{1, q} \subset \mathrm{U}_{1}^{\text {ssp }} \cap \Omega .
$$

2. First step $(q=2)$. From Proposition 18, for each $\ell \in\left\{1, \ldots, \mathrm{N}_{1}\right\}, \mathrm{E}_{1, \ell} \cap \mathrm{U}_{0}^{\Omega} \neq\left\{x_{1, \ell}\right\}$ if and only if $\mathrm{U}_{1}^{\mathrm{ssp}} \cap \mathrm{E}_{1, \ell} \neq \emptyset$. As a consequence, one has:

$$
\mathrm{U}_{1}^{\text {ssp }} \bigcap\left(\cup_{\ell=1}^{\mathrm{N}_{1}} \mathrm{E}_{1, \ell}\right) \neq \emptyset \text { iff }\left\{x_{1,1}, \ldots, x_{1, \mathrm{~N}_{1}}\right\} \neq \mathrm{U}_{0}^{\Omega}
$$

If $U_{1}^{\text {ssp }} \cap\left(\cup_{\ell=1}^{N_{1}} E_{1, \ell}\right)=\emptyset$ (or equivalently $N_{1}=m_{0}^{\Omega}$ ), the constructions of the maps $\widetilde{\mathbf{j}}$ and $\mathbf{j}$ are finished and one goes to item 4 below. If $U_{1}^{\text {ssp }} \cap\left(\cup_{\ell=1}^{N_{1}} E_{1, \ell}\right) \neq \emptyset$ (or equivalently $N_{1}<m_{0}^{\Omega}$ ), one defines

$$
\sigma_{2}:=\max _{x \in \cup_{1}^{\text {ssp }} \cap\left(\cup_{\ell=1}^{N_{1}} E_{1, \ell}\right)} f(x) \in\left(\min _{\cup_{\ell=1}^{N_{1}} \mathrm{E}_{1, \ell}} f, \max _{\ell \in\left\{1, \ldots, \mathrm{N}_{1}\right\}} \sigma_{1, \ell}\right)
$$

The set

$$
\bigcup_{\ell=1}^{\mathrm{N}_{1}}\left(\mathrm{E}_{1, \ell} \cap\left\{f<\sigma_{2}\right\}\right)
$$

is then the union of finitely many connected components. We denote by $\mathrm{E}_{2,1}, \ldots, \mathrm{E}_{2, \mathrm{~N}_{2}}$ (with $\mathrm{N}_{2} \geq$ 1) the connected components of $\bigcup_{\ell=1}^{N_{1}}\left(E_{1, \ell} \cap\left\{f<\sigma_{2}\right\}\right)$ which do not contain any of the minima $\left\{x_{1,1}, \ldots, x_{1, N_{1}}\right\}$. From items 1 and 2 in Proposition 18 (applied to $C=E_{1, \ell} \cap\left\{f<\sigma_{2}\right\}$ for each $\ell \in\left\{1, \ldots, N_{1}\right\}$,

$$
\forall \ell \in\left\{1, \ldots, \mathrm{N}_{2}\right\}, \mathrm{E}_{2, \ell} \in \mathcal{C}_{\text {crit }} .
$$

Notice that the other connected components (i.e. those containing the $x_{1, \ell}$ 's) may be not critical. Let us associate with each $\mathrm{E}_{2, \ell}, 1 \leq \ell \leq \mathrm{N}_{2}$, one point $x_{2, \ell}$ arbitrarily chosen in $\arg \min _{\overline{\mathrm{E}_{2, \ell}}} f=\arg \min _{\mathrm{E}_{2, \ell}} f$ (the last equality follows from the fact that $\partial \mathrm{E}_{2, \ell} \subset\left\{f=\sigma_{2}\right\}$ ). For $\ell \in\left\{1, \ldots, \mathrm{N}_{2}\right\}$, let us define: 


$$
\widetilde{\mathbf{j}}\left(x_{2, \ell}\right):=\mathrm{E}_{2, \ell} \text { and } \mathbf{j}\left(x_{2, \ell}\right):=\partial \mathrm{E}_{2, \ell} \cap \mathrm{U}_{1}^{\text {ssp }} \subset\left\{f=\sigma_{2}\right\}
$$

3. Recurrence $(q \geq 3)$. If all the local minima of $f$ in $\Omega$ have been labeled at the end of the previous step above $\left(q=2\right.$ ), i.e. if $\cup_{j=1}^{2}\left\{x_{j, 1}, \ldots, x_{j, \mathrm{~N}_{j}}\right\}=\mathrm{U}_{0}^{\Omega}$ (or equivalently if $\mathrm{N}_{1}+\mathrm{N}_{2}=\mathrm{m}_{0}^{\Omega}$ ), the constructions of the maps $\widetilde{\mathbf{j}}$ and $\mathbf{j}$ are finished and one goes to item 4 below. If it is not the case, from Proposition 18, there exists $m \in \mathbb{N}^{*}$ such that

$$
\forall q \in\{2, \ldots, m+1\}, \mathrm{U}_{1}^{\mathrm{ssp}} \bigcap \bigcup_{\ell=1}^{\mathrm{N}_{1}}\left(\mathrm{E}_{1, \ell} \cap\left\{f<\sigma_{q}\right\}\right) \neq \emptyset
$$

where one defines recursively the decreasing sequence $\left(\sigma_{q}\right)_{q=3, \ldots, m+2}$ by

$$
\sigma_{q}:=\max \left\{f(x), x \in \mathrm{U}_{1}^{\mathrm{ssp}} \bigcap \bigcup_{\ell=1}^{\mathrm{N}_{1}}\left(\mathrm{E}_{1, \ell} \cap\left\{f<\sigma_{q-1}\right\}\right)\right\} .
$$

Notice that $\sigma_{q} \in\left(\min _{\cup_{\ell=1}^{N_{1}} \mathrm{E}_{1, \ell}} f, \sigma_{q-1}\right)$. Let us now consider the largest integer $m^{*} \in \mathbb{N}^{*}$ such that (43) holds. Notice that $m^{*}$ is well defined since the cardinal of $\mathrm{U}_{0}^{\Omega}$ is finite. By definition of $m^{*}$, one has:

$$
\mathrm{U}_{1}^{\mathrm{ssp}} \bigcap \bigcup_{\ell=1}^{\mathrm{N}_{1}}\left(\mathrm{E}_{1, \ell} \cap\left\{f<\sigma_{m^{*}+2}\right\}\right)=\emptyset
$$

Then, one repeats recursively $m^{*}$ times the procedure described in the first step above. For $q \in$ $\left\{2, \ldots, m^{*}+1\right\}$, one defines $\left(\mathrm{E}_{q+1, \ell}\right)_{\ell \in\left\{1, \ldots, \mathrm{N}_{q+1}\right\}}$ as the set of connected components of $\bigcup_{\ell=1}^{\mathrm{N}_{1}}\left(\mathrm{E}_{1, \ell} \cap\{f<\right.$ $\left.\left.\sigma_{q+1}\right\}\right)$ which does not contain any of the local minima $\cup_{j=1}^{q}\left\{x_{j, 1}, \ldots, x_{j, N_{j}}\right\}$ of $f$ in $\Omega$ which have been previously chosen. From items 1 and 2 in Proposition $18, \forall \ell \in\left\{1, \ldots, \mathrm{N}_{q+1}\right\}, \mathrm{E}_{q+1, \ell} \in \mathcal{C}_{\text {crit }}$. For $\ell \in$ $\left\{1, \ldots, \mathrm{N}_{q+1}\right\}$, we associate with each $\mathrm{E}_{q+1, \ell}$, one point $x_{q+1, \ell} \operatorname{arbitrarily~chosen~in~} \arg \min _{\mathrm{E}_{q+1, \ell}} f$. For $\ell \in\left\{1, \ldots, \mathrm{N}_{q+1}\right\}$, let us define:

$$
\widetilde{\mathbf{j}}\left(x_{q+1, \ell}\right):=\mathrm{E}_{q+1, \ell} \text { and } \mathbf{j}\left(x_{q+1, \ell}\right):=\partial \mathrm{E}_{q+1, \ell} \cap \mathrm{U}_{1}^{\text {ssp }} \subset\left\{f=\sigma_{q+1}\right\} \text {. }
$$

From (44) and Proposition 18, $\mathrm{U}_{0}^{\Omega}=\cup_{j=1}^{m^{*}+2}\left\{x_{j, 1}, \ldots, x_{j, \mathrm{~N}_{j}}\right\}$ and thus, all the local minima of $f$ in $\Omega$ are labeled. This concludes the constructions of the maps $\widetilde{\mathbf{j}}$ and $\mathbf{j}$.

4. Properties of the maps $\widetilde{\mathbf{j}}$ and $\mathbf{j}$. The two maps

$$
\widetilde{\mathbf{j}}: \mathrm{U}_{0}^{\Omega} \longrightarrow \mathcal{C}_{\text {crit }} \quad \text { and } \quad \mathbf{j}: \mathrm{U}_{0}^{\Omega} \longrightarrow \mathcal{P}\left(\mathrm{U}_{1}^{\bar{\Omega}}\right)
$$

are clearly injective. Notice that the $\mathbf{j}(x), x \in \mathrm{U}_{0}^{\Omega}$, are not disjoint in general. For all $x \in \mathrm{U}_{0}^{\Omega}, f(\mathbf{j}(x))$ contains exactly one value, which will be denoted by $f(\mathbf{j}(x))$. Moreover, since $\cup_{\ell=1}^{\mathrm{N}_{1}} \mathrm{E}_{1, \ell} \subset \Omega$ (see the first statement in (38)), one has for all $x \in \mathrm{U}_{0}^{\Omega}, \widetilde{\mathbf{j}}(x) \subset \Omega$. Moreover, it holds

$$
\forall x \in \mathrm{U}_{0}^{\Omega} \backslash\left\{x_{1,1}, \ldots, x_{1, \mathrm{~N}_{1}}\right\}, \mathbf{j}(x) \subset \Omega \cap \mathrm{U}_{1}^{\text {ssp }}
$$

Finally, for all $x \in \mathbf{U}_{0}^{\Omega}, f(\mathbf{j}(x))-f(x)>0$ and for all $x \in \mathbf{U}_{0}^{\Omega} \cap \widetilde{\mathbf{j}}\left(x_{1, \ell}\right) \backslash\left\{x_{1, \ell}\right\}$

$$
f(\mathbf{j}(x))-f(x)<\min _{\ell=1, \ldots, \mathrm{N}_{1}} f\left(\mathbf{j}\left(x_{1, \ell}\right)\right)-f\left(x_{1, \ell}\right) .
$$




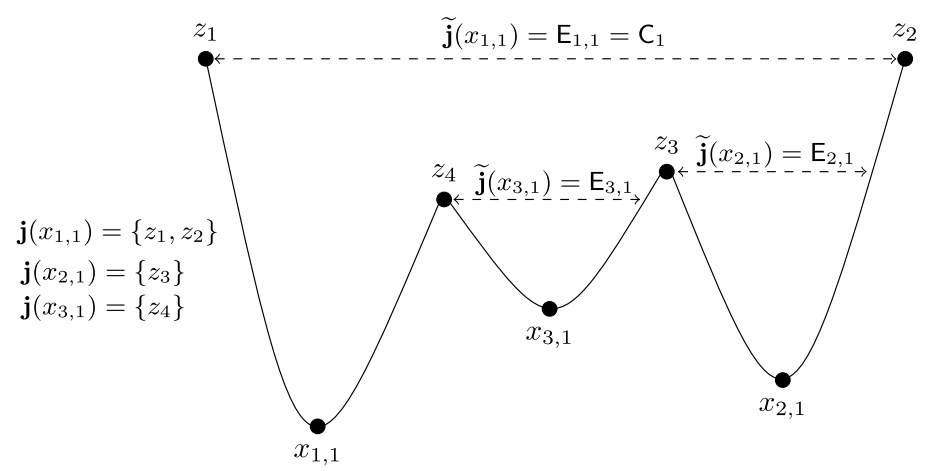

Fig. 4. The maps $\mathbf{j}$ and $\tilde{\mathbf{j}}$ on a one-dimensional example for which the maps are uniquely defined and the construction requires three steps.

The constructions of the maps $\mathbf{j}$ and $\widetilde{\mathbf{j}}$ are illustrated in Fig. 4 on a one-dimensional example. Since one can pick a minimum or another in a critical connected component at each step of the construction of $\mathbf{j}$ and $\widetilde{\mathbf{j}}$, the maps are not uniquely defined if over one of the connected components $\mathrm{E}_{k, \ell}\left(k \geq 1, \ell \in\left\{1, \ldots, \mathrm{N}_{k}\right\}\right)$, $\arg \min f$ contains more than one point (see [24, Fig. 9] for a one-dimensional example). As will become clear below, this non-uniqueness has no influence on the results proven hereafter.

Remark 19. In the case when for all local minima $x$ of $f, \mathbf{j}(x)$ is a single point, $\mathbf{j}(x) \cap \mathbf{j}(y)=\emptyset$ for all $x \neq y$ and when all the heights $(f(\mathbf{j}(x))-f(x))_{x \in \mathrm{U}_{1}^{\bar{\Omega}}}$ are distinct, the map $\mathbf{j}$ is exactly the one constructed in [23].

The next definition will be used in Section 3.2 to construct the quasi-modes.

Definition 20. Let us assume that (A0) is satisfied. Let $\varepsilon$ be such that

$$
0 \leq \varepsilon<\min _{k \geq 1, \ell \in\left\{1, \ldots, \mathrm{N}_{k}\right\}}\left(\max _{\overline{\mathrm{E}}_{k, \ell}} f-\max _{\mathrm{U}_{1}^{\mathrm{sp}} \cap \mathrm{E}_{k, \ell}} f\right),
$$

where the family $\left(\mathrm{E}_{k, \ell}\right)_{k \geq 1, \ell \in\left\{1, \ldots, \mathrm{N}_{k}\right\}}$ is defined in the construction of the map $\mathbf{j}$ above. For $k \geq 1$ and $\ell \in\left\{1, \ldots, \mathrm{N}_{k}\right\}$, one defines

$$
\mathrm{E}_{k, \ell}(\varepsilon)=\mathrm{E}_{k, \ell} \cap\left\{f<\max _{\overline{\mathrm{E}}_{k, \ell}} f-\varepsilon\right\}
$$

which is a connected component of $\left\{f<\max _{\overline{\mathrm{E}}_{k, \ell}} f-\varepsilon\right\}$ according to item 1 in Proposition 18.

\subsection{Rewriting the assumptions (A1)-(A4) in terms of the map $\mathbf{j}$}

Lemma 21. Let us assume that (A0) is satisfied. Then, the assumption (A1) is equivalent to the fact that there exists $\ell \in\left\{1, \ldots, N_{1}\right\}$ such that for all $k \in\left\{1, \ldots, N_{1}\right\} \backslash\{\ell\}$,

$$
f\left(\mathbf{j}\left(x_{1, k}\right)\right)-f\left(x_{1, k}\right)<f\left(\mathbf{j}\left(x_{1, \ell}\right)\right)-f\left(x_{1, \ell}\right) .
$$

Thus, when (A1) holds, the elements of $\mathcal{C}=\left\{\mathrm{C}_{1}, \ldots, \mathrm{C}_{\mathrm{N}_{1}}\right\}$ (see Definition 12) are ordered such that $\ell=1$, i.e. for all $k \in\left\{2, \ldots, \mathrm{N}_{1}\right\}$ :

$$
f\left(\mathbf{j}\left(x_{1, k}\right)\right)-f\left(x_{1, k}\right)<f\left(\mathbf{j}\left(x_{1,1}\right)\right)-f\left(x_{1,1}\right) .
$$

With this numbering, one has $\mathrm{C}_{1}=\mathrm{C}_{\max }$, where $\mathrm{C}_{\max }$ is defined in (A1). 
Proof. Assume that the hypothesis (A0) is satisfied. Let us recall that $\mathcal{C}=\left\{\mathrm{C}(x), x \in \mathrm{U}_{0}^{\Omega}\right\}=\left\{\mathrm{C}_{1}, \ldots, \mathrm{C}_{\mathrm{N}_{1}}\right\}$. Let $\mathrm{C} \in \mathcal{C}$ and let $k \in\left\{1, \ldots, \mathrm{N}_{1}\right\}$, such that $\mathrm{C}=\mathrm{C}_{k}$. Then, from (33) and the first step of the construction of $\mathbf{j}$ in Section 2.3, one has for all $q \in\left\{1, \ldots, \mathrm{N}_{1}\right\}: \mathrm{H}_{f}\left(x_{1, q}\right)=\lambda\left(x_{1, q}\right)=f\left(\mathbf{j}\left(x_{1, q}\right)\right)=\sup _{\mathrm{C}_{q}} f$ and $f\left(x_{1, q}\right)=\min _{\overline{C_{q}}} f$. Thus, it holds $\sup _{\mathrm{C}} f-\min _{\overline{\mathrm{C}}} f=f\left(\mathbf{j}\left(x_{1, k}\right)\right)-f\left(x_{1, k}\right)$. This implies the results stated in Lemma 21.

In view of Lemma 21 and by construction of the map $\mathbf{j}$ (see the first step in Section 2.3), one can rewrite the assumptions (A1), (A2), (A3), and (A4) with the map $\mathbf{j}$ as follows:

- The assumption (A1) is equivalent to the fact that, up to reordering the elements of $\mathcal{C}=\left\{\mathrm{C}_{1}, \ldots, \mathrm{C}_{\mathrm{N}_{1}}\right\}$ such that (50) is satisfied, it holds:

$$
\forall x \in\left\{x_{1,2}, \ldots, x_{1, \mathrm{~N}_{1}}\right\}, f(\mathbf{j}(x))-f(x)<f\left(\mathbf{j}\left(x_{1,1}\right)\right)-f\left(x_{1,1}\right)
$$

Furthermore, in this case, $C_{1}=C_{\max }$, where $C_{\max }$ is defined by (A1).

- The assumption (A2) rewrites when (A1j) holds,

$$
\partial \mathrm{C}_{1} \cap \partial \Omega \neq \emptyset .
$$

- The assumption (A3) rewrites when (A1j) holds,

$$
\partial \mathrm{C}_{1} \cap \partial \Omega \subset \underset{\partial \Omega}{\arg \min } f .
$$

- When (A1j) holds, the assumption (A4) is equivalent to

$$
\mathbf{j}\left(x_{1,1}\right) \subset \partial \Omega .
$$

This equivalence between (A4) and ( $\mathbf{A} 4 \mathbf{j})$ follows from $(\mathbf{A} 1 \mathbf{j})$ together with the fact that $\mathbf{j}\left(x_{1,1}\right)=\partial \mathrm{C}_{1} \cap \mathrm{U}_{1}^{\text {ssp }}$ (see (41)) and by definition of a separating saddle point. From now on, we work with the formulations (A1j), (A2j), (A3j), and (A4j) of the assumptions (A1), (A2), (A3), and (A4).

For one-dimensional illustrations of the assumptions, we refer to [24, Figures 6 to 9]. Notice that un$\operatorname{der}(\mathbf{A} 1 \mathbf{j})$, it holds from (47):

$$
\forall x \in \mathbf{U}_{0}^{\Omega} \backslash\left\{x_{1,1}\right\}, f(\mathbf{j}(x))-f(x)<f\left(\mathbf{j}\left(x_{1,1}\right)\right)-f\left(x_{1,1}\right) .
$$

When (A1j) and (A2j) are satisfied, from Definition 13 and Proposition 15 (see the first inclusion in (39) and (41)), one has

$$
\partial \Omega \cap \mathbf{j}\left(x_{1,1}\right)=\partial \Omega \cap \partial \mathrm{C}_{1}=\mathrm{U}_{1}^{\partial \Omega} \cap \partial \mathrm{C}_{1} .
$$

In that case, we assume that the elements $\left\{z_{1}, \ldots, z_{\mathrm{m}_{1}^{\partial \Omega}}\right\}$ of $\mathrm{U}_{1}^{\partial \Omega}$ (see (20)) are ordered such that

$$
\partial \Omega \cap \partial \mathrm{C}_{1}=\left\{z_{1}, \ldots, z_{\mathrm{k}_{1}} \mathrm{c}_{1}\right\}
$$

where $\mathrm{k}_{1}^{\partial \mathrm{C}_{1}} \in \mathbb{N}^{*}$ satisfies $\mathrm{k}_{1}^{\partial \mathrm{C}_{1}} \leq \mathrm{m}_{1}^{\partial \Omega}$ (see (20)). Notice that from Lemma 21, this labeling implies when (A3j) is satisfied:

$$
\mathrm{k}_{1}^{\partial \mathrm{C}_{1}}=\mathrm{k}_{1}^{\partial \mathrm{C}_{\max }}
$$

where $k_{1}^{\partial C_{\max }}$ is defined by (23). Let us finally prove the following result which will be used in the sequel. 
Lemma 22. Let us assume that (A0), (A1j), (A2j), and (A3j) hold. Then, one has $\min _{\Omega} f=\min _{\bar{\Omega}} f<$ $\min _{\partial \Omega} f$ and

$$
\underset{\mathrm{C}_{1}}{\arg \min } f=\underset{\bar{\Omega}}{\arg \min } f .
$$

Proof. The fact that $\min _{\bar{\Omega}} f<\min _{\partial \Omega} f$ is obvious. Let us prove (55). Let $k \in\left\{1, \ldots, \mathrm{N}_{1}\right\}$ and let us recall that from Definition 12, there exists $x \in \mathrm{U}_{0}^{\Omega} \cap \mathrm{C}_{k}$ such that $\mathrm{C}_{k}=\mathrm{C}(\lambda(x), x)$. Let us assume that $x \in \arg \min _{\Omega} f$. Then, by definition of the map $\mathbf{j}$ and by definition of $\lambda(x)$ (see (32)) together with the fact that (A1j), (A2j), and (A3j) hold, one has $f\left(\mathbf{j}\left(x_{1, k}\right)\right)=\lambda(x) \geq \min _{\partial \Omega} f=f\left(\mathbf{j}\left(x_{1,1}\right)\right)$. Thus, if $f\left(x_{1, k}\right)=$ $f(x) \leq f\left(x_{1,1}\right)$, it holds $\lambda(x)-f(x)=f\left(\mathbf{j}\left(x_{1, k}\right)\right)-f\left(x_{1, k}\right) \geq f\left(\mathbf{j}\left(x_{1,1}\right)\right)-f\left(x_{1,1}\right)$. This implies $\mathrm{C}_{k}=\mathrm{C}_{1}$ from the assumption $(\mathbf{A} 1 \mathbf{j})$. This concludes the proof of $(55)$.

\section{Constructions of the quasi-modes}

This section is dedicated to the constructions of two families of quasi-modes approximating the eigenforms of $-L_{f, h}^{D,(0)}$ and $-L_{f, h}^{D,(1)}$ associated with exponentially small eigenvalues. These constructions use the maps $\mathbf{j}$ and $\mathbf{j}$ introduced in the previous section.

This section is organized as follows. In Section 3.1, we introduce the notations used throughout this paper for operators, and the properties of Witten Laplacians and of the operators $L_{f, h}^{D,(p)}$ needed in our analysis. The quasi-modes are then built in Section 3.2.

\subsection{Notations and Witten Laplacian}

\subsubsection{Notation for Sobolev spaces}

For $p \in\{0, \ldots, d\}$, one denotes by $\Lambda^{p} C^{\infty}(\bar{\Omega})$ the space of $C^{\infty} p$-forms on $\bar{\Omega}$. Moreover, $\Lambda^{p} C_{T}^{\infty}(\bar{\Omega})$ is the set of $C^{\infty} p$-forms $v$ such that $\mathbf{t} v=0$ on $\partial \Omega$, where $\mathbf{t}$ denotes the tangential trace on forms. The weighted space $\Lambda^{p} L_{w}^{2}(\Omega)$ is the completion of $\Lambda^{p} C^{\infty}(\bar{\Omega})$ for the norm

$$
w \in \Lambda^{p} C^{\infty}(\bar{\Omega}) \mapsto \sqrt{\int_{\Omega}|w|^{2} e^{-\frac{2}{h} f}} .
$$

Likewise, for $p \in\{0, \ldots, d\}$ and $q \in \mathbb{N}, \Lambda^{p} H_{w}^{q}(\Omega)$ is the weighted Sobolev spaces of $p$-forms on $\Omega$ with regularity index $q: v \in \Lambda^{p} H_{w}^{q}(\Omega)$ if and only if for every multi-index $\alpha$ with $|\alpha| \leq q, \partial^{\alpha} v$ is in $\Lambda^{p} L_{w}^{2}(\Omega)$. See for example [33] for an introduction to Sobolev spaces on manifolds with boundaries. For $p \in\{0, \ldots, d\}$ and $q>\frac{1}{2}$, the set $\Lambda^{p} H_{w, T}^{q}(\Omega)$ is defined by

$$
\Lambda^{p} H_{w, T}^{q}(\Omega):=\left\{v \in \Lambda^{p} H_{w}^{q}(\Omega) \mid \mathbf{t} v=0 \text { on } \partial \Omega\right\}
$$

Notice that $\Lambda^{p} L_{w}^{2}(\Omega)$ is the space $\Lambda^{p} H_{w}^{0}(\Omega)$, and that $\Lambda^{0} H_{w, T}^{1}(\Omega)$ is the space $H_{w, 0}^{1}(\Omega)$ already introduced in Proposition 4 . We will denote by $\|\cdot\|_{H_{w}^{q}}$ the norm on the weighted space $\Lambda^{p} H_{w}^{q}(\Omega)$. Moreover $\langle\cdot, \cdot\rangle_{L_{w}^{2}}$ denotes the scalar product in $\Lambda^{p} L_{w}^{2}(\Omega)$. Finally, we will also use the same notation without the index $w$ to denote the standard Sobolev spaces defined with respect to the Lebesgue measure on $\Omega$.

\subsubsection{The Witten Laplacian and the infinitesimal generator of the diffusion (1)}

In this section, we recall some basic properties of Witten Laplacians, as well as the link between those and the operators $L_{f, h}^{(p)}$ introduced above (see (7) and (29)). 
For $p \in\{0, \ldots, n\}$, one defines the distorted exterior derivative $\grave{a}$ la Witten $d_{f, h}^{(p)}: \Lambda^{p} C^{\infty}(\Omega) \rightarrow$ $\Lambda^{p+1} C^{\infty}(\Omega)$ and its formal adjoint: $d_{f, h}^{(p) *}: \Lambda^{p+1} C^{\infty}(\Omega) \rightarrow \Lambda^{p} C^{\infty}(\Omega)$ by

$$
d_{f, h}^{(p)}:=e^{-\frac{1}{h} f} h d^{(p)} e^{\frac{1}{h} f} \text { and } d_{f, h}^{(p) *}:=e^{\frac{1}{h} f} h d^{(p) *} e^{-\frac{1}{h} f} .
$$

The Witten Laplacian, firstly introduced in [34], is then defined similarly as the Hodge Laplacian $\Delta_{H}^{(p)}:=$ $\left(d+d^{*}\right)^{2}$ by

$$
\Delta_{f, h}^{(p)}:=\left(d_{f, h}+d_{f, h}^{*}\right)^{2}=d_{f, h} d_{f, h}^{*}+d_{f, h}^{*} d_{f, h}: \Lambda^{p} C^{\infty}(\Omega) \rightarrow \Lambda^{p} C^{\infty}(\Omega)
$$

The Dirichlet realization of $\Delta_{f, h}^{(p)}$ on $\Lambda^{p} L^{2}(\Omega)$ is denoted by $\Delta_{f, h}^{D,(p)}$ and its domain is

$$
D\left(\Delta_{f, h}^{D,(p)}\right)=\left\{w \in \Lambda^{p} H^{2}(\Omega) \mid \mathbf{t} w=0, \mathbf{t} d_{f, h}^{*} w=0\right\}
$$

The operator $\Delta_{f, h}^{D,(p)}$ is self-adjoint, nonnegative, and its associated quadratic form is given by

$$
\phi \in \Lambda^{p} H_{T}^{1}(\Omega) \mapsto\left\|d_{f, h}^{(p)} \phi\right\|_{L^{2}}^{2}+\left\|d_{f, h}^{(p) *} \phi\right\|_{L^{2}}^{2},
$$

where

$$
\Lambda^{p} H_{T}^{1}(\Omega)=\left\{w \in \Lambda^{p} H^{1}(\Omega) \mid \mathbf{t} w=0\right\}
$$

We refer in particular to [23, Section 2.4] for a comprehensive definition of Witten Laplacians with Dirichlet tangential boundary conditions and statements on their properties. The link between the Witten Laplacian and the infinitesimal generator $L_{f, h}^{(0)}$ of the diffusion (1) is the following: since

$$
L_{f, h}^{(0)}=-\nabla f \cdot \nabla-\frac{h}{2} \Delta_{H}^{(0)} \text { and } \Delta_{f, h}^{(0)}=h^{2} \Delta_{H}^{(0)}+|\nabla f|^{2}+h \Delta_{H}^{(0)} f,
$$

one has:

$$
\Delta_{f, h}^{D,(0)}=-2 h U L_{f, h}^{D,(0)} U^{-1}
$$

where $U$ is the unitary operator

$$
U:\left\{\begin{array}{l}
\Lambda^{p} L_{w}^{2}(\Omega) \rightarrow \Lambda^{p} L^{2}(\Omega) \\
\phi \mapsto e^{-\frac{1}{h} f} \phi .
\end{array}\right.
$$

In particular, the operator $L_{f, h}^{D,(0)}$ has a natural extension to $p$-forms defined by the relation

$$
L_{f, h}^{D,(p)}=-\frac{1}{2 h} U^{-1} \Delta_{f, h}^{D,(p)} U
$$

For $p=1$, one recovers the operator $L_{f, h}^{(1)}$ with tangential Dirichlet boundary conditions defined by (28)-(29). The operator $L_{f, h}^{D,(p)}$ with domain

$$
D\left(L_{f, h}^{D,(p)}\right)=U^{-1} D\left(\Delta_{f, h}^{D,(p)}\right)=\left\{w \in \Lambda^{p} H_{w}^{2}(\Omega) \mid \mathbf{t} w=0, \mathbf{t} d_{\frac{2 f}{h}, 1}^{*} w=0\right\}
$$


is then self-adjoint on $\Lambda^{p} L_{w}^{2}(\Omega)$, non positive and its associated quadratic form is

$$
\Lambda^{p} H_{T}^{1}(\Omega) \ni \phi \mapsto-\frac{h}{2}\left[\left\|d^{(p)} \phi\right\|_{L_{w}^{2}}^{2}+\left\|d_{\frac{2 f}{h}, 1}^{(p) *} \phi\right\|_{L_{w}^{2}}^{2}\right] .
$$

Let us also recall that $-L_{f, h}^{D,(p)}$ (and equivalently $\Delta_{f, h}^{D,(p)}$ ) has a compact resolvent. From general results on elliptic operators when $p=0,-L_{f, h}^{D,(0)}$ (and $\Delta_{f, h}^{D,(0)}$ ) admits a non degenerate smallest eigenvalue with an associated eigenfunction which has a sign on $\Omega$. Denoting moreover by $\pi_{E}\left(L_{f, h}^{D,(p)}\right)$ the spectral projector associated with $L_{f, h}^{D,(p)}$ and some Borel set $E \subset \mathbb{R}$, the following commutation relations hold on $\Lambda^{p} H_{T}^{1}(\Omega)$ :

$$
d^{(p)} \pi_{E}\left(L_{f, h}^{D,(p)}\right)=\pi_{E}\left(L_{f, h}^{D,(p+1)}(\Omega)\right) d^{(p)} \text { and } d_{\frac{2 f}{h}, 1}^{(p) *} \pi_{E}\left(L_{f, h}^{D,(p)}\right)=\pi_{E}\left(L_{f, h}^{D,(p-1)}\right) d_{\frac{2 f}{h}, 1}^{(p) *} .
$$

Let us recall that from the elliptic regularity of $L_{f, h}^{D,(p)}$, for any bounded Borel set $E \subset \mathbb{R}, \operatorname{Ran} \pi_{E}\left(L_{f, h}^{D,(d)}\right) \subset$ $\Lambda^{p} C_{T}^{\infty}(\bar{\Omega})$, the relation (59) then leads to the following complex structure:

$$
\{0\} \longrightarrow \operatorname{Ran} \pi_{E}\left(L_{f, h}^{D,(0)}\right) \stackrel{d_{f, h}}{\longrightarrow} \operatorname{Ran} \pi_{E}\left(L_{f, h}^{D,(1)}\right) \stackrel{d_{f, h}}{\longrightarrow} \cdots \stackrel{d_{f, h}}{\longrightarrow} \operatorname{Ran} \pi_{E}\left(L_{f, h}^{D,(d)}\right) \stackrel{d_{f, h}}{\longrightarrow}\{0\}
$$

and

$$
\{0\} \stackrel{d_{\frac{2 f}{h}, 1}^{*}}{\longleftarrow} \operatorname{Ran} \pi_{E}\left(L_{f, h}^{D,(0)}\right) \stackrel{d_{\frac{2 f}{h}, 1}^{*}}{\longleftarrow} \operatorname{Ran} \pi_{E}\left(L_{f, h}^{D,(1)}\right) \stackrel{d_{\frac{2 f}{h}, 1}^{*}}{\longleftarrow} \cdots \stackrel{d_{\frac{2 f}{h}, 1}^{*}}{\longleftarrow} \operatorname{Ran} \pi_{E}\left(L_{f, h}^{D,(d)}\right) \longleftarrow\{0\} .
$$

For ease of notation, one defines:

$$
\forall p \in\{0, \ldots, d\}, \quad \pi_{h}^{(p)}:=\pi_{\left[0, \frac{\sqrt{h}}{2}\right)}\left(-L_{f, h}^{D,(p)}\right) .
$$

The following result, instrumental in our investigation of the smallest eigenvalue $\lambda_{h}$ of $-L_{f, h}^{D,(0)}$, is an immediate consequence of [23, Theorem 3.2.3] together with (58).

Lemma 23. Under assumption (A0), there exists $h_{0}>0$ such that for all $h \in\left(0, h_{0}\right)$,

$$
\operatorname{dim} \operatorname{Ran} \pi_{h}^{(0)}=\mathrm{m}_{0}^{\Omega} \text { and } \operatorname{dim} \operatorname{Ran} \pi_{h}^{(1)}=\mathrm{m}_{1}^{\bar{\Omega}},
$$

where $\mathrm{m}_{0}^{\Omega}=\operatorname{Card}\left(\mathrm{U}_{0}^{\Omega}\right)$ and $\mathrm{m}_{1}^{\bar{\Omega}}=\operatorname{Card}\left(\mathrm{U}_{1}^{\bar{\Omega}}\right)$ are defined in Section 1.3.2.

Remark 24. In [23, Theorem 3.2.3] it is assumed that $f: \partial \Omega \rightarrow \mathbb{R}$ is a Morse function while in (A0), we only assume that $f:\left\{x \in \partial \Omega, \partial_{n} f(x)>0\right\} \rightarrow \mathbb{R}$ is a Morse function. As mentioned in [35, Section 7.1], the statement of Lemma 23 still holds under this weaker assumption. This is explained in details in [24, Appendix A].

In the sequel, with a slight abuse of notation, one denotes the exterior differential $d$ acting on functions by $\nabla$. Note that it follows from the above considerations and Lemma 23 that under (A0), it holds

$$
u_{h} \in \operatorname{Ran} \pi_{h}^{(0)} \text { and } \nabla u_{h} \in \operatorname{Ran} \pi_{h}^{(1)} .
$$

Moreover, from (58), it is equivalent to study the spectrum of $L_{f, h}^{D,(0)}$ or the spectrum of $\Delta_{f, h}^{D,(0)}$. We end this section with the following standard lemma which will be frequently used throughout this work (see for instance [36, Theorem 8.15]). 
Lemma 25. Let $(A, D(A))$ be a non negative self-adjoint operator on a Hilbert space $(\mathcal{H},\|\cdot\|)$ with associated quadratic form $q_{A}(x)=(x, A x)$ whose domain is $Q(A)$. It then holds, for any $u \in Q(A)$ and $b>0$,

$$
\left\|\pi_{[b,+\infty)}(A) u\right\|^{2} \leq \frac{q_{A}(u)}{b}
$$

where, for a Borel set $E \subset \mathbb{R}, \pi_{E}(A)$ is the spectral projector associated with $A$ and $E$.

3.2. Construction of the quasi-modes for $-L_{f, h}^{D,(0)}$ and $-L_{f, h}^{D,(1)}$

Let us recall that from Lemma 23, one has for any $h$ small enough $\operatorname{dim} \operatorname{Ran} \pi_{h}^{(0)}=\mathrm{m}_{0}^{\Omega}$ and $\operatorname{dim} \operatorname{Ran} \pi_{h}^{(1)}=$ $\mathrm{m}_{1}^{\bar{\Omega}}$, where $\mathrm{m}_{0}^{\Omega}$ is the number of local minima of $f$ in $\Omega$ and $\mathrm{m}_{1}^{\bar{\Omega}}$ is the number of generalized saddle points of $f$ in $\bar{\Omega}$, see Section 1.3.2. To prove Theorem 1, the strategy consists in constructing a family of $\mathrm{m}_{0}^{\Omega}$ quasi-modes in order to approximate $\operatorname{Ran} \pi_{h}^{(0)}$ and a family of $\mathrm{m}_{1}^{\bar{\Omega}}$ quasi-modes in order to approximate $\operatorname{Ran} \pi_{h}^{(1)}$ (see (60) for the definitions of the spectral projectors $\pi_{h}^{(p)}$ ).

Since the construction of the quasi-modes rely on the one made for Witten Laplacians in [31,23,28], we first construct quasi-modes for the Witten Laplacians $\Delta_{f, h}^{D,(0)}$ in Section 3.2.1, and $\Delta_{f, h}^{D,(1)}$ in Section 3.2.2. The quasi-modes for $-L_{f, h}^{D,(0)}$ and $-L_{f, h}^{D,(1)}$ are then obtained using (58) in Section 3.2.3.

\subsubsection{Quasi-modes for the Witten Laplacian $\Delta_{f, h}^{D,(0)}$}

Let us assume that (A0) is satisfied. Let us recall that from Lemma 23 and (58), there exists $h_{0}>0$ such that for any $h \in\left(0, h_{0}\right), \operatorname{dim} \operatorname{Ran} \pi_{\left[0, h \frac{3}{2}\right)}\left(\Delta_{f, h}^{D,(0)}\right)=\mathrm{m}_{0}^{\Omega}$. In this section, the maps $\mathbf{j}$ and $\widetilde{\mathbf{j}}$ introduced in Section 2.3 are used to build a family of $\mathrm{m}_{0}^{\Omega}$ functions whose span approximates $\operatorname{Ran} \pi_{\left[0, h^{\frac{3}{2}}\right)}\left(\Delta_{f, h}^{D,(0)}\right)$. The properties of this family which are listed in this section will be useful to prove Proposition 43 below and Propositions 46 and 47 in the next section. Following [31,23,28], each critical point $x \in \mathbf{U}_{0}^{\Omega}$ is associated with a quasi-mode for $\Delta_{f, h}^{D,(0)}$. The notation follows the one introduced in Section 2.3.

Let us first introduce two parameters $\varepsilon_{1}>0$ and $\varepsilon>0$ which will be used to define the quasi-modes for $\Delta_{f, h}^{D,(0)}$. In the following, $d$ is the geodesic distance on $\bar{\Omega}$ for the initial metric. Let us consider $\varepsilon_{1}>0$ small enough such that

$$
\forall z, z^{\prime} \in \mathrm{U}_{1}^{\bar{\Omega}}, z \neq z^{\prime} \text { implies } d\left(z, z^{\prime}\right) \geq 6 \varepsilon_{1}
$$

and for all $z \in \mathrm{U}_{1}^{\bar{\Omega}}$ (thanks to (37)),

$$
\begin{aligned}
& \text { either } z \in \mathrm{U}_{1}^{\Omega} \text { and }\{f<f(z)\} \cap B\left(z, 2 \varepsilon_{1}\right) \text { has two connected components, } \\
& \text { or } z \in \mathrm{U}_{1}^{\partial \Omega} \text { and }\{f<f(z)\} \cap B\left(z, 2 \varepsilon_{1}\right) \text { is connected. }
\end{aligned}
$$

The parameter $\varepsilon_{1}>0$ will be successively reduced a finite number of times in this section and in Section 3.2.2, and it will be kept fixed from the end of Section 3.2.2.

Let $\varepsilon>0$ be such that

$$
0<\varepsilon<\frac{1}{2} \min _{k \geq 1, \ell \in\left\{1, \ldots, \mathrm{N}_{k}\right\}}\left(\max _{\overline{\mathrm{E}}_{k, \ell}} f-\max _{\mathrm{U}_{1}^{\mathrm{sp}} \cap \mathrm{E}_{k, \ell}} f\right)
$$

which ensures in particular that $\mathrm{E}_{k, \ell}(\varepsilon)$ and $\mathrm{E}_{k, \ell}(2 \varepsilon)$ are connected for all $k \geq 1$ and $\ell \in\left\{1, \ldots, \mathrm{N}_{1}\right\}$, see (49). The parameter $\varepsilon>0$ will be further reduced a finite number of times in the following sections so that $\partial\left\{\chi_{k, \ell}^{\varepsilon, \varepsilon_{1}}=1\right\}$ is as close as necessary to $\partial \mathrm{E}_{k, \ell}$ near $\mathbf{j}\left(x_{k, \ell}\right)$, where the cut-off function $\chi_{k, \ell}^{\varepsilon, \varepsilon_{1}}$ is introduced in the next definition of the quasi-mode for $\Delta_{f, h}^{D,(0)}$ associated to $x_{k, \ell}$. 
Definition 26. Let us assume that (A0) holds. For $k \geq 1$ and $\ell \in\left\{1, \ldots, \mathrm{N}_{k}\right\}$, the quasi-mode associated with $x_{k, \ell}$ is defined by:

$$
\forall \ell \in\left\{1, \ldots, \mathbf{N}_{k}\right\}, \widetilde{v}_{k, \ell}:=\frac{\chi_{k, \ell}^{\varepsilon, \varepsilon_{1}} e^{-\frac{1}{h} f}}{\left\|\chi_{k, \ell}^{\varepsilon, \varepsilon_{1}} e^{-\frac{1}{h} f}\right\|_{L^{2}}},
$$

where the functions $\chi_{k, \ell}^{\varepsilon, \varepsilon_{1}} \in C_{c}^{\infty}\left(\Omega, \mathbb{R}^{+}\right)$satisfy the following properties: there exists $\varepsilon_{1}^{0}>0$ such that for all $\varepsilon_{1} \in\left(0, \varepsilon_{1}^{0}\right]$, there exists $\varepsilon^{0}>0$ such that for all $\varepsilon \in\left(0, \varepsilon^{0}\right]$,

a) It holds

$$
\left\{\begin{array}{l}
\overline{\mathrm{E}_{k, \ell}(2 \varepsilon)} \subset\left\{\chi_{k, \ell}^{\varepsilon, \varepsilon_{1}}=1\right\} \text { and } \\
\operatorname{supp} \chi_{k, \ell}^{\varepsilon, \varepsilon_{1}} \subset\left\{x \in \Omega, d\left(x, \overline{\mathrm{E}_{k, \ell}}\right) \leq 3 \varepsilon_{1}\right\} \backslash \mathbf{j}\left(x_{k, \ell}\right),
\end{array}\right.
$$

see (35) for the definition of $\mathrm{E}_{k, \ell}$ and (49) for the definition of $\mathrm{E}_{k, \ell}(2 \varepsilon)$.

b) For all $y \in \operatorname{supp} \chi_{k, \ell}^{\varepsilon, \varepsilon_{1}}$,

$$
f(y) \leq f\left(\mathbf{j}\left(x_{k, \ell}\right)\right) \text { implies } y \in \overline{\mathrm{E}_{k, \ell}}
$$

and hence, according to (66),

$$
\left\{\begin{array}{l}
\arg \min _{\operatorname{supp}} \chi_{k, \ell}^{\varepsilon, \varepsilon_{1}} f=\arg \min _{\mathrm{E}_{k, \ell}} f \text { and } \\
\min _{\text {supp } \nabla \chi_{k, \ell}^{\varepsilon, \varepsilon_{1}}} f \geq f\left(\mathbf{j}\left(x_{k, \ell}\right)\right)-2 \varepsilon
\end{array}\right.
$$

c) For all $z \in \mathbf{j}\left(x_{k, \ell}\right) \cap \Omega$, it holds

$$
\operatorname{supp} \chi_{k, \ell}^{\varepsilon, \varepsilon_{1}} \cap B\left(z, 2 \varepsilon_{1}\right) \neq \emptyset \text { and } \operatorname{supp} \chi_{k, \ell}^{\varepsilon, \varepsilon_{1}} \cap B\left(z, 2 \varepsilon_{1}\right) \subset \mathrm{E}_{k, \ell} .
$$

d) For all $z \in \mathrm{U}_{1}^{\bar{\Omega}} \backslash \mathbf{j}\left(x_{k, \ell}\right)$, it holds

$$
\left\{\begin{array}{l}
z \in \overline{\mathrm{E}_{k, \ell}} \text { and } \overline{B\left(z, 2 \varepsilon_{1}\right)} \subset\left\{\chi_{k, \ell}^{\varepsilon, \varepsilon_{1}}=1\right\} \text { or } \\
z \notin \overline{\mathrm{E}_{k, \ell}} \text { and } \overline{B\left(z, 2 \varepsilon_{1}\right)} \subset\left\{\chi_{k, \ell}^{\varepsilon, \varepsilon_{1}}=0\right\}
\end{array}\right.
$$

e) For all $q \in\left\{1, \ldots, N_{k}\right\} \backslash\{\ell\}$, it holds $\operatorname{supp} \chi_{k, q}^{\varepsilon, \varepsilon_{1}} \cap \operatorname{supp} \chi_{k, \ell}^{\varepsilon, \varepsilon_{1}}=\emptyset$.

f) For $k \geq 2$ and for any $\left(k^{\prime}, \ell^{\prime}\right) \in\{1, \ldots, k-1\} \times\left\{1, \ldots, \mathrm{N}_{k^{\prime}}\right\}$ such that $\mathrm{E}_{k, \ell} \subset \mathrm{E}_{k^{\prime}, \ell^{\prime}}$, it holds

$$
\operatorname{supp} \chi_{k, \ell}^{\varepsilon, \varepsilon_{1}} \subset\left\{\chi_{k^{\prime}, \ell^{\prime}}^{\varepsilon, \varepsilon_{1}}=1\right\}
$$

Notice that by a connexity argument, it holds $\mathrm{E}_{k, \ell} \subset \mathrm{E}_{k^{\prime}, \ell^{\prime}}$ or $\mathrm{E}_{k, \ell} \cap \mathrm{E}_{k^{\prime}, \ell^{\prime}}=\emptyset$.

In Figs. 5 and 6 , for $k \geq 1$, one gives a schematic representation of the cut-off function $\chi_{k, \ell}^{\varepsilon, \varepsilon_{1}}$ near $z \in \mathrm{U}_{1}^{\bar{\Omega}}$ in the three situations: (i) $k=1, \ell \in\left\{1, \ldots, \mathrm{N}_{1}\right\}$ and $z \in \mathbf{j}\left(x_{1, \ell}\right) \cap \partial \Omega$; (ii) $\ell \in\left\{1, \ldots, \mathrm{N}_{k}\right\}$ and $z \in \mathbf{j}\left(x_{k, \ell}\right) \cap \Omega$; (iii) $\ell \in\left\{1, \ldots, \mathrm{N}_{k}\right\}, z \in\left(\mathrm{U}_{1}^{\bar{\Omega}} \backslash \mathbf{j}\left(x_{k, \ell}\right)\right) \cap \partial \mathrm{E}_{k, \ell}$.

For the ease of notation, we do not indicate explicitly the dependence on the parameters $\varepsilon$ and $\varepsilon_{1}$ in the notation of the functions $\widetilde{v}_{k, \ell}$ for $k \geq 1, \ell \in\left\{1, \ldots, \mathrm{N}_{k}\right\}$. The following lemma will be useful to estimate $\left\|\left(1-\pi_{h}^{(0)}\right) \widetilde{v}_{k, \ell}\right\|_{L^{2}}$ when $h \rightarrow 0$, for $k \geq 1$ and $\ell \in\left\{1, \ldots, \mathrm{N}_{k}\right\}$, see indeed item 2a in Proposition 43 below. 

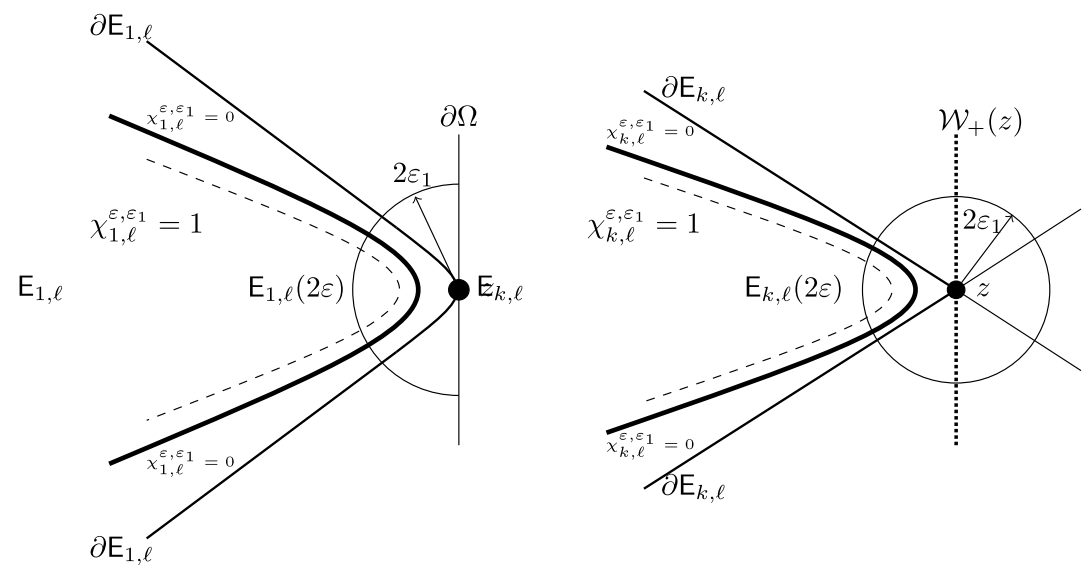

Fig. 5. Schematic representation of the cut-off function $\chi_{k, \ell}^{\varepsilon, \varepsilon_{1}}$. Left: near $z \in \mathbf{j}\left(x_{1, \ell}\right) \cap \partial \Omega \subset \mathrm{U}_{1}^{\partial \Omega} \cap \mathrm{U}_{1}^{\text {ssp }}$ for $\ell \in\left\{1, \ldots, \mathrm{N}_{1}\right\}$. Right: near $z \in \mathbf{j}\left(x_{k, \ell}\right) \cap \Omega \subset \mathrm{U}_{1}^{\Omega}$ for $k \geq 1$ and $\ell \in\left\{1, \ldots, \mathrm{N}_{k}\right\}\left(\mathcal{W}_{+}\right.$is the stable manifold of the saddle point $\left.z\right)$. The point $z$ is a separating saddle point as introduced in Definition 13. The dashed line represents $\partial \mathrm{E}_{k, \ell}(2 \varepsilon)$ and the thick solid line represents $\operatorname{supp} \nabla \chi_{k, \ell}^{\varepsilon, \varepsilon_{1}}$.

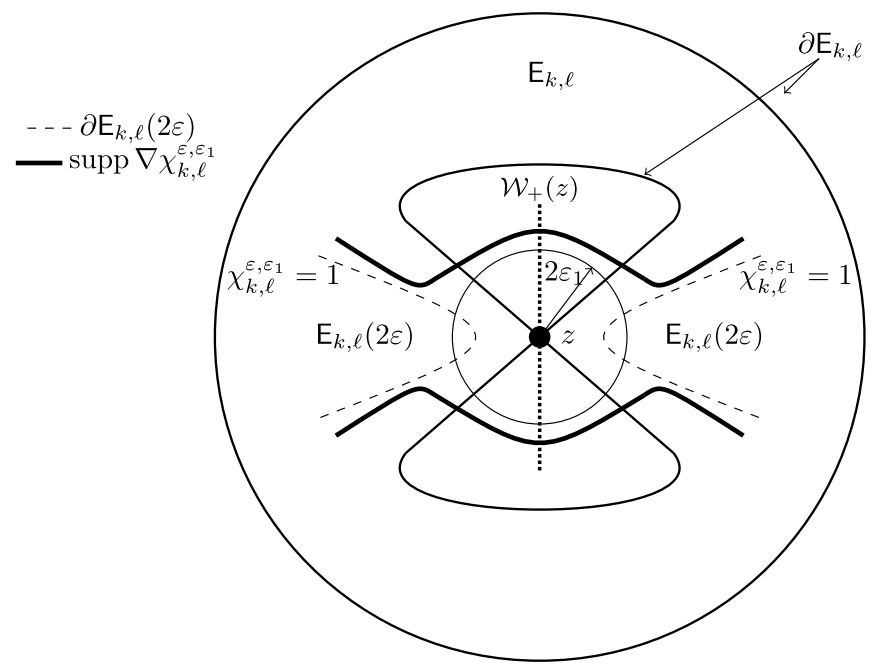

Fig. 6. Schematic representation of the cut-off function $\chi_{k, \ell}^{\varepsilon, \varepsilon_{1}}$ near $z \in\left(\mathbf{U}_{1}^{\bar{\Omega}} \backslash \mathbf{j}\left(x_{k, \ell}\right)\right) \cap \partial \mathrm{E}_{k, \ell}$. The point $z$ is a saddle point on $\partial \mathrm{E}_{k, \ell}$ but is not a separating saddle point as introduced in Definition 13.

Lemma 27. Let us assume that (A0) holds. Then, for $k \geq 1$ and $\ell \in\left\{1, \ldots, \mathrm{N}_{k}\right\}$, there exist $c>0, C>0$ and $h_{0}>0$ such that for all $h \in\left(0, h_{0}\right)$,

$$
\left\|d_{f, h} \widetilde{v}_{k, \ell}\right\|_{L^{2}}=\frac{\left\|h e^{-\frac{1}{h} f} d \chi_{k, \ell}^{\varepsilon, \varepsilon_{1}}\right\|_{L^{2}}}{\left\|e^{-\frac{1}{h} f} \chi_{k, \ell}^{\varepsilon, \varepsilon_{1}}\right\|_{L^{2}}} \leq C e^{-\frac{1}{h}\left(f\left(\mathbf{j}\left(x_{k, \ell}\right)\right)-f\left(x_{k, \ell}\right)-c \varepsilon\right)}
$$

where the function $\widetilde{v}_{k, \ell}$ has been introduced in Definition 26.

Proof. This estimate follows from (65)-(67), and Laplace's method applied to $\left\|\chi_{k, \ell}^{\varepsilon, \varepsilon_{1}} e^{-\frac{1}{h} f}\right\|_{L^{2}}$.

The following lemma ensures that the family $\left(\widetilde{v}_{k, \ell}\right)_{k \geq 1, \ell \in\left\{1, \ldots, \mathrm{N}_{k}\right\}}$ is uniformly linearly independent for any $h$ small enough (the proof of this result is made in [28, Section 4.2]).

Lemma 28. Let us assume that (A0) holds. The family $\mathcal{B}_{v}=\left(\widetilde{v}_{k, \ell}\right)_{k \geq 1, \ell \in\left\{1, \ldots, \mathrm{N}_{k}\right\}}$ introduced in Definition 26, is linearly independent, uniformly with respect to $h$ small enough: for some (and hence for any) orthonormal 
(for the $L^{2}$-scalar product) family $\mathcal{B}_{o}$ spanning $\operatorname{Span}\left(\mathcal{B}_{v}\right)$, for any matrix norm $\|\cdot\|$ on $\mathbb{R}^{\mathrm{m}_{0}^{\Omega} \times \mathrm{m}_{0}^{\Omega}}$, there exist $C>0$ and $h_{0}>0$ such that for all $h \in\left(0, h_{0}\right)$,

$$
\left\|\operatorname{Mat}_{\mathcal{B}_{o}} \mathcal{B}_{v}\right\| \leq C \quad \text { and } \quad\left\|\operatorname{Mat}_{\mathcal{B}_{v}} \mathcal{B}_{o}\right\| \leq C
$$

\subsubsection{Quasi-modes for the Witten Laplacian $\Delta_{f, h}^{D,(1)}$}

Let us assume that (A0) is satisfied. Let us recall that from Lemma 23 and (58), there exists $h_{0}>0$ such that for any $h \in\left(0, h_{0}\right), \operatorname{dim} \operatorname{Ran} \pi_{\left[0, h^{\frac{3}{2}}\right)}\left(\Delta_{f, h}^{D,(1)}\right)=\mathrm{m}_{1}^{\bar{\Omega}}$. In this section, a family of 1 -forms $\left(\widetilde{\phi}_{j}\right)_{j \in\left\{1, \ldots, \mathrm{m}_{1}^{\bar{\Omega}}\right\}}$ approximating $\operatorname{Ran} \pi_{\left[0, h^{\frac{3}{2}}\right)}\left(\Delta_{f, h}^{D,(1)}\right)$ is built. To this end, for each $z \in \mathrm{U}_{1}^{\bar{\Omega}}$, one constructs a 1-form locally supported in a neighborhood of $z$ in $\bar{\Omega}$, following the procedure in $[31,28]$ if $z \in \mathrm{U}_{1}^{\Omega}$, and the procedure in [23] if $z \in \mathrm{U}_{1}^{\partial \Omega}$. For the sake of completeness, we recall these constructions, and we provide associated estimates which will be used throughout this work.

Quasi-mode associated with $z \in \mathrm{U}_{1}^{\Omega}$. Let us recall that from $(21), \mathrm{U}_{1}^{\Omega}=\left\{z_{\mathrm{m}_{1}^{\partial \Omega}+1}, \ldots, z_{\mathrm{m}_{1}^{\bar{\Omega}}}\right\} \subset \Omega$ is the set of saddle points of $f$ in $\Omega$. Let $j \in\left\{\mathrm{m}_{1}^{\partial \Omega}+1, \ldots, \mathrm{m}_{1}^{\bar{\Omega}}\right\}$ and $z_{j} \in \mathrm{U}_{1}^{\Omega}$. Let $\mathcal{V}_{j}$ be some small smooth neighborhood of $z_{j}$ such that $\overline{\mathcal{V}_{j}} \cap \partial \Omega=\emptyset$ and for $x \in \overline{\mathcal{V}_{j}},|\nabla f(x)|=0$ if and only if $x=z_{j}$. Let us now consider the full Dirichlet realization $\Delta_{f, h}^{F D,(1)}\left(\mathcal{V}_{j}\right)$ of the Witten Laplacian $\Delta_{f, h}^{(1)}$ in $\mathcal{V}_{j}$ whose domain is

$$
D\left(\Delta_{f, h}^{F D,(1)}\left(\mathcal{V}_{j}\right)\right)=\left\{w \in \Lambda^{1} H^{2}\left(\mathcal{V}_{j}\right),\left.w\right|_{\partial \mathcal{V}_{j}}=0\right\}
$$

Let us recall that according to [32, Section 2], there exists, choosing if necessary $\mathcal{V}_{j}$ smaller, a $C^{\infty}$ non negative solution $\Phi_{j}: \mathcal{V}_{j} \rightarrow \mathbb{R}^{+}$to the eikonal equation

$$
\left|\nabla \Phi_{j}\right|=|\nabla f| \text { in } \mathcal{V}_{j} \quad \text { such that } \Phi_{j}(y)=0 \text { iff } y=z_{j}
$$

Moreover, $\Phi_{j}$ is the unique non negative solution to $(73)$ in the sense that if $\widetilde{\Phi}_{j}: \widetilde{\mathcal{V}}_{j} \rightarrow \mathbb{R}^{+}$is another non negative $C^{\infty}$ solution to (73) on a neighborhood $\widetilde{\mathcal{V}_{j}}$ of $z_{j}$, then $\widetilde{\Phi}_{j}=\Phi_{j}$ on $\widetilde{\mathcal{V}_{j}} \cap \mathcal{V}_{j}$.

Remark 29. The function $\Phi_{j}$ is actually the Agmon distance to $z_{j}$, i.e. $\Phi_{j}$ is the distance to $z_{j}$ in $\bar{\Omega}$ associated with the metric $|\nabla f|^{2} d x^{2}$, where $d x^{2}$ is the Riemannian metric on $\bar{\Omega}$ (see [32, Section 1]).

The next proposition, which follows from [32, Theorem 1.4 and Lemma 1.6], gathers all the estimates one needs in the following on the operator $\Delta_{f, h}^{F D,(1)}\left(\mathcal{V}_{j}\right)$.

Proposition 30. Let us assume that $(\mathbf{A 0})$ is satisfied. Then, the operator $\Delta_{f, h}^{F D,(1)}\left(\mathcal{V}_{j}\right)$ is self-adjoint, has compact resolvent and is positive. Moreover:

- There exist $\varepsilon_{0}>0$ and $h_{0}>0$ such that for all $h \in\left(0, h_{0}\right)$ :

$$
\operatorname{dim} \operatorname{Ran} \pi_{\left[0, \varepsilon_{0} h\right)}\left(\Delta_{f, h}^{F D,(1)}\left(\mathcal{V}_{j}\right)\right)=1
$$

- The smallest eigenvalue $\lambda_{h}\left(\mathcal{V}_{j}\right)$ of $\Delta_{f, h}^{F D,(1)}\left(\mathcal{V}_{j}\right)$ is exponentially small: there exist $C>0, c>0$ and $h_{0}>0$ such that for any $h \in\left(0, h_{0}\right)$ :

$$
\lambda_{h}\left(\mathcal{V}_{j}\right) \leq C e^{-\frac{c}{h}}
$$

- Any $L^{2}$-normalized eigenform $w_{j}$ associated with the smallest eigenvalue $\lambda_{h}\left(\mathcal{V}_{j}\right)$ of $\Delta_{f, h}^{F D,(1)}\left(\mathcal{V}_{j}\right)$ satisfies the following Agmon estimate: for all $\varepsilon>0$, there exist $C_{\varepsilon}>0$ and $h_{0}>0$ such that for any $h \in\left(0, h_{0}\right)$, it holds: 


$$
\left\|e^{\frac{1}{h} \Phi_{j}} w_{j}\right\|_{H^{1}\left(\mathcal{V}_{j}\right)} \leq C_{\varepsilon} e^{\frac{\varepsilon}{h}}
$$

where $\Phi_{j}$ is the solution to (73).

Choosing $\varepsilon_{1}$ smaller if necessary, one may assume that there exists $\alpha>0$ such that $B\left(z_{j}, 2 \varepsilon_{1}+\alpha\right) \subset \mathcal{V}_{j}$. Let us now define the quasi-mode associated with $z_{j} \in \mathrm{U}_{1}^{\Omega}$.

Definition 31. Let us assume that (A0) is satisfied. Let $j \in\left\{\mathrm{m}_{1}^{\partial \Omega}+1, \ldots, \mathrm{m}_{1}^{\bar{\Omega}}\right\}$ and $z_{j} \in \mathrm{U}_{1}^{\Omega}$. The quasi-mode associated with $z_{j}$ is defined by

$$
\widetilde{\phi}_{j}:=\frac{\theta_{j} w_{j}}{\left\|\theta_{j} w_{j}\right\|_{L^{2}}} \in \Lambda^{1} C_{c}^{\infty}(\Omega)
$$

where $w_{j}$ is a $L^{2}$-normalized eigenform associated with the smallest eigenvalue $\lambda_{h}\left(\mathcal{V}_{j}\right)$ of $\Delta_{f, h}^{F D,(1)}\left(\mathcal{V}_{j}\right)$ and $\theta_{j}$ is a smooth non negative cut-off function satisfying, $\operatorname{supp} \theta_{j} \subset B\left(z_{j}, 2 \varepsilon_{1}\right) \subset \mathcal{V}_{j}$ and $\theta_{j}=1$ on $B\left(z_{j}, \varepsilon_{1}\right)$.

Notice that both $w_{j}$ and $-w_{j}$ can be used to build a quasi-mode: the choice of the sign will be determined in Proposition 33 below. Moreover, using (76) together with the fact that for all $j \in\left\{\mathrm{m}_{1}^{\partial \Omega}+1, \ldots, \mathrm{m}_{1}^{\bar{\Omega}}\right\}$, $\inf _{\text {supp }\left(1-\theta_{j}\right) \cap \mathcal{V}_{j}} \Phi_{j}>0($ see $(73))$, one has when $h \rightarrow 0$ :

$$
\left\|\left(1-\theta_{j}\right) w_{j}\right\|_{L^{2}\left(\mathcal{V}_{j}\right)}=O\left(e^{-\frac{c}{h}}\right) \text { and therefore, }\left\|\theta_{j} w_{j}\right\|_{L^{2}}=1+O\left(e^{-\frac{c}{h}}\right),
$$

for some $c>0$ independent of $h$. Using Proposition 30 and (78), one deduces the following estimate on the quasi-mode $\widetilde{\phi}_{j}$ introduced in Definition 31.

Corollary 32. Let us assume that (A0) holds. Let $\widetilde{\phi}_{j}$ be the quasi-mode associated with $z_{j} \in U_{1}^{\Omega}(j \in$ $\left.\left\{\mathrm{m}_{1}^{\partial \Omega}+1, \ldots, \mathrm{m}_{1}^{\bar{\Omega}}\right\}\right)$, see Definition 31. Then, there exist $C>0, c>0$ and $h_{0}>0$ such that for any $h \in\left(0, h_{0}\right)$ :

$$
\left\|d_{f, h} \widetilde{\phi}_{j}\right\|_{L^{2}}+\left\|d_{f, h}^{*} \widetilde{\phi}_{j}\right\|_{L^{2}} \leq C e^{-\frac{c}{h}}
$$

Let us now recall the construction of a WKB approximation of $w_{j}$ made in [32] and which will be needed in the following. Let us denote by $\mathcal{W}_{+}\left(z_{j}\right)$ and $\mathcal{W}_{-}\left(z_{j}\right)$ respectively the stable and unstable manifolds of $z_{j}$ associated with the flow of $-\nabla f$ which are defined as follows. Denoting by $\varphi_{t}(y)$ the solution of $\frac{d}{d t} \varphi_{t}(y)=-\nabla f\left(\varphi_{t}(y)\right)$ with initial condition $\varphi_{0}(y)=y$,

$$
\mathcal{W}_{ \pm}\left(z_{j}\right):=\left\{y \in \Omega, \varphi_{t}(y) \underset{t \rightarrow \pm \infty}{\longrightarrow} z_{j}\right\}
$$

It then holds (see indeed [32, Section 2] and [31, Section 4.2]): $\operatorname{dim} \mathcal{W}_{+}\left(z_{j}\right)=d-1, \operatorname{dim} \mathcal{W}_{-}\left(z_{j}\right)=1$, and for all $y \in \mathcal{V}_{j}$ (assuming $\mathcal{V}_{j}$ small enough),

$$
\left|f(y)-f\left(z_{j}\right)\right| \leq \Phi_{j}(y) \text { and }\left|f(y)-f\left(z_{j}\right)\right|=\Phi_{j}(y) \text { iff } y \in \mathcal{W}_{+}\left(z_{j}\right) \cup \mathcal{W}_{-}\left(z_{j}\right)
$$

with moreover

$$
\Phi_{j}= \pm\left(f-f\left(z_{j}\right)\right) \text { on } \mathcal{W}_{ \pm}\left(z_{j}\right) \text { and } \operatorname{det} \operatorname{Hess} \Phi_{j}\left(z_{j}\right)=\left|\operatorname{det} \operatorname{Hess} f\left(z_{j}\right)\right|
$$

Additionally, there exists from [32, Proposition 1.3 and Section 2] a $C^{\infty}\left(\overline{\mathcal{V}_{j}}\right)$ 1-form $a_{j}(x, h)=\widetilde{a}_{j}(x)+O(h)$ such that $a_{j}\left(z_{j}, h\right)=\widetilde{a}_{j}\left(z_{j}\right)=n\left(z_{j}\right)$, where $n\left(z_{j}\right)$ is a unit normal to $\mathcal{W}_{+}\left(z_{j}\right)$, and such that the 1 -form $u_{j, w k b}^{(1)}=a_{j} e^{-\frac{1}{h} \Phi_{j}}$ satisfies 


$$
\left(\Delta_{f, h}^{(1)}-\mu(h)\right) u_{j, w k b}^{(1)}=O\left(h^{\infty}\right) e^{-\frac{1}{h} \Phi_{j}} \text { in } \mathcal{V}_{j}
$$

where $\mu(h) \sim h^{2} \sum_{k=0}^{\infty} \mu_{k} h^{k}$. Moreover, one has in the limit $h \rightarrow 0$ (see [32, Section 2]):

$$
\left\|\theta_{j} u_{j, w k b}^{(1)}\right\|_{L^{2}}=\frac{(\pi h)^{\frac{d}{4}}}{\left|\operatorname{det} \operatorname{Hess} f\left(z_{j}\right)\right|^{\frac{1}{4}}}(1+O(h)),
$$

where the remainder term $O(h)$ admits a full asymptotic expansion in $h$. Using in addition the fact that $\Phi_{j}>0$ on $\operatorname{supp} \nabla \theta_{j}$, there exists $c>0$ such that for $h$ small enough:

$$
\left\|\left(\Delta_{f, h}^{(1)}-\mu(h)\right)\left(\theta_{j} u_{j, w k b}^{(1)}\right)\right\|_{L^{2}}=O\left(h^{\infty}\right)+O\left(e^{-\frac{c}{h}}\right)=O\left(h^{\infty}\right) .
$$

From (83), one then obtains that $\Delta_{f, h}^{F D,(1)}\left(\mathcal{V}_{j}\right)$ admits an eigenvalue which equals $\mu(h)+O\left(h^{\infty}\right)$. Since $\mu(h)=O\left(h^{2}\right)$, from (74) and (75), one deduces that $\mu_{k}=0$ for all $k \geq 0$ and thus $\mu(h)=O\left(h^{\infty}\right)$. Finally, one has:

$$
\Delta_{f, h}^{(1)} u_{j, w k b}^{(1)}=O\left(h^{\infty}\right) e^{-\frac{1}{h} \Phi_{j}} \text { in } \mathcal{V}_{j}
$$

In the following proposition, $w_{j}$ and $u_{j, w k b}^{(1)}$ are compared.

Proposition 33. Let us assume that (A0) is satisfied. Let $w_{j}$ be a $L^{2}$-normalized eigenform associated with the smallest eigenvalue $\lambda_{h}\left(\mathcal{V}_{j}\right)$ of $\Delta_{f, h}^{F D,(1)}\left(\mathcal{V}_{j}\right)\left(j \in\left\{\mathrm{m}_{1}^{\partial \Omega}+1, \ldots, \mathrm{m}_{1}^{\bar{\Omega}}\right\}\right)$. Then, there exists $h_{0}>0$ such that for all $h \in\left(0, h_{0}\right)$ one has:

$$
\left\|\theta_{j}\left(w_{j}-c_{j}(h) u_{j, w k b}^{(1)}\right)\right\|_{H^{1}}=O\left(h^{\infty}\right)
$$

where $c_{j}(h)^{-1}=\left\langle w_{j}, \theta_{j} u_{j, w k b}^{(1)}\right\rangle_{L^{2}}$. In addition, up to replacing $w_{j}$ by $-w_{j}$, one can assume that $c_{j}(h) \geq 0$ for $h$ small enough and then, in the limit $h \rightarrow 0$, one has:

$$
c_{j}(h)=\frac{\left|\operatorname{det} \operatorname{Hess} f\left(z_{j}\right)\right|^{\frac{1}{4}}}{(\pi h)^{\frac{d}{4}}}(1+O(h)),
$$

where the remainder term $O(h)$ admits a full asymptotic expansion in $h$.

Proof. Let us define $k_{j}(h):=\left\langle w_{j}, \theta_{j} u_{j, w k b}^{(1)}\right\rangle_{L^{2}}=c_{j}(h)^{-1}$. If $k_{j}(h)<0$, then one changes $w_{j}$ to $-w_{j}$ so that one can suppose without loss of generality that $k_{j}(h) \geq 0$. For $h$ small enough, one has from (74) $\pi_{\left[0, \varepsilon_{0} h\right)}\left(\Delta_{f, h}^{F D,(1)}\left(\mathcal{V}_{j}\right)\right)\left(\theta_{j} u_{j, w k b}^{(1)}\right)=k_{j}(h) w_{j}$. Let us define the following 1 -form $\alpha_{j}:=\theta_{j}\left(u_{j, w k b}^{(1)}-k_{j}(h) w_{j}\right)$. Thus, the following identity holds for $h$ small enough

$$
\alpha_{j}=k_{j}(h)\left(1-\theta_{j}\right) w_{j}+\pi_{\left[\varepsilon_{0} h,+\infty\right)}\left(\Delta_{f, h}^{F D,(1)}\left(\mathcal{V}_{j}\right)\right)\left(\theta_{j} u_{j, w k b}^{(1)}\right) .
$$

Notice that, from (83), there exist $C>0$ and $h_{0}>0$ such that for all $h \in\left(0, h_{0}\right)\left|k_{j}(h)\right| \leq C h^{\frac{d}{4}}$. Therefore, using Lemma 25, (78), and (84), there exist $c>0$ and $C>0$ such that for $h$ small enough:

$$
\begin{aligned}
\left\|\alpha_{j}\right\|_{L^{2}}^{2} & \leq 2 k_{j}(h)^{2}\left\|\left(1-\theta_{j}\right) w_{j}\right\|_{L^{2}}^{2}+2\left\|\pi_{\left[\varepsilon_{0} h,+\infty\right)}\left(\Delta_{f, h}^{F D,(1)}\left(\mathcal{V}_{j}\right)\right)\left(\theta_{j} u_{j, w k b}^{(1)}\right)\right\|_{L^{2}}^{2} \\
& \leq C h^{\frac{d}{2}} e^{-\frac{c}{h}}+C h^{-1} O\left(h^{\infty}\right)=O\left(h^{\infty}\right) .
\end{aligned}
$$


Moreover, since $d_{f, h}=h d+d f \wedge$ and $d_{f, h}^{*}=h d^{*}+\mathbf{i}_{\nabla f}$, one obtains using the Gaffney inequality (see [33, Corollary 2.1.6]):

$$
\begin{aligned}
\left\|\alpha_{j}\right\|_{H^{1}}^{2} & \leq C\left(\left\|d \alpha_{j}\right\|_{L^{2}}^{2}+\left\|d^{*} \alpha_{j}\right\|_{L^{2}}^{2}+\left\|\alpha_{j}\right\|_{L^{2}}^{2}\right) \\
& \leq C h^{-2}\left(\left\|d_{f, h} \alpha_{j}\right\|_{L^{2}}^{2}+\left\|d_{f, h}^{*} \alpha_{j}\right\|_{L^{2}}^{2}+\left\|\alpha_{j}\right\|_{L^{2}}^{2}\right) .
\end{aligned}
$$

Furthermore, from (79), it holds $\left\|d_{f, h}\left(\theta_{j} w_{j}\right)\right\|_{L^{2}}+\left\|d_{f, h}^{*}\left(\theta_{j} w_{j}\right)\right\|_{L^{2}} \leq C e^{-\frac{c}{h}}$ and from (84) $\left\|d_{f, h}\left(\theta_{j} u_{j, w k b}^{(1)}\right)\right\|_{L^{2}}$ $+\left\|d_{f, h}^{*}\left(\theta_{j} u_{j, w k b}^{(1)}\right)\right\|_{L^{2}}=O\left(h^{\infty}\right)$. Thus, there exists $C>0$ such that:

$$
\left\|\alpha_{j}\right\|_{H^{1}}^{2}=O\left(h^{\infty}\right)
$$

This concludes the proof of (85). Finally, since $\left\|\theta_{j} w_{j}\right\|_{L^{2}}=1+O\left(e^{-\frac{c}{h}}\right)$ (see (78)), by considering $\| \theta_{j}\left(u_{j, w k b}^{(1)}-\right.$ $\left.k_{j}(h) w_{j}\right) \|_{L^{2}}^{2}=O\left(h^{\infty}\right)$, one gets using (83):

$$
k_{j}(h)^{2}=\frac{\left\|\theta_{j} u_{j, w k b}^{(1)}\right\|_{L^{2}}^{2}+O\left(h^{\infty}\right)}{2-\left\|\theta_{j} w_{j}\right\|_{L^{2}}^{2}}=\frac{(\pi h)^{\frac{d}{2}}}{\left|\operatorname{det} \operatorname{Hess} f\left(z_{j}\right)\right|^{\frac{1}{2}}}(1+O(h)) .
$$

Since $k_{j}(h) \geq 0$, one has $k_{j}(h)=\frac{(\pi h)^{\frac{d}{4}}}{\left|\operatorname{det} \operatorname{Hess} f\left(z_{j}\right)\right|^{\frac{1}{4}}}(1+O(h))$. This concludes the proof of $(86)$ since $c_{j}(h)=$ $k_{j}(h)^{-1}$.

Quasi-mode associated with $z \in \mathrm{U}_{1}^{\partial \Omega}$. Let us recall that from $(20), \mathrm{U}_{1}^{\partial \Omega}=\left\{z_{1}, \ldots, z_{\mathrm{m}_{1}^{\partial \Omega}}\right\} \subset \partial \Omega$ is the set of generalized saddle points on $\partial \Omega$. Let $j \in\left\{1, \ldots, \mathrm{m}_{1}^{\partial \Omega}\right\}$ and $z_{j} \in \mathrm{U}_{1}^{\partial \Omega}$. To construct a 1-form locally supported in a neighborhood of $z_{j}$ in $\bar{\Omega}$, one proceeds in the same way as in [23, Section 4.3]. Let $\mathcal{V}_{j}$ be a small neighborhood of $z_{j}$ in $\bar{\Omega}$ such that $\mathcal{V}_{j}$ satisfies: $|\nabla f|>0$ on $\overline{\mathcal{V}_{j}}$, for all $x \in \partial \mathcal{V}_{j} \cap \partial \Omega,\left|\nabla_{T} f(x)\right|=0$ if and only if $x=z_{j}$, and $\partial_{n} f>0$ on $\partial \Omega \cap \partial \mathcal{V}_{j}$. Let us now consider the mixed full Dirichlet-tangential Dirichlet realization $\Delta_{f, h}^{M D,(1)}\left(\mathcal{V}_{j}\right)$ of the Witten Laplacian $\Delta_{f, h}^{(1)}$ in $\mathcal{V}_{j}$ whose domain is

$$
D\left(\Delta_{f, h}^{M D,(1)}\left(\mathcal{V}_{j}\right)\right)=\left\{w \in \Lambda^{1} H^{2}\left(\mathcal{V}_{j}\right),\left.w\right|_{\partial \mathcal{V}_{j} \cap \Omega}=0,\left.\mathbf{t} w\right|_{\partial \mathcal{V}_{j} \cap \partial \Omega}=0 \text { and }\left.\mathbf{t} d_{f, h}^{*} w\right|_{\partial \mathcal{V}_{j} \cap \partial \Omega}=0\right\}
$$

see [23, Remark 4.3.1] for the characterization of its domain. Since $\partial_{n} f>0$ on $\partial \Omega \cap \mathcal{V}_{j}$, from [23, Section 4.2], one has that, choosing $\mathcal{V}_{j}$ small enough, there exists a $C^{\infty}\left(\mathcal{V}_{j}, \mathbb{R}^{+}\right)$non negative solution $\Phi_{j}$ to the eikonal equation

$$
\left\{\begin{array}{l}
\left|\nabla \Phi_{j}\right|=|\nabla f| \text { in } \Omega \cap \mathcal{V}_{j} \\
\Phi_{j}=f-f\left(z_{j}\right) \text { on } \partial \Omega \cap \mathcal{V}_{j} \\
\partial_{n} \Phi_{j}=-\partial_{n} f \text { on } \partial \Omega \cap \mathcal{V}_{j}
\end{array}\right.
$$

Moreover, up to choosing $\mathcal{V}_{j}$ small enough

$$
\Phi_{j}(y)=0 \text { iff } y=z_{j} .
$$

Besides, $\Phi_{j}$ is the unique non negative solution to $(87)$ in the sense that if $\widetilde{\Phi}_{j}: \widetilde{\mathcal{V}}_{j} \rightarrow \mathbb{R}^{+}$is another non negative $C^{\infty}$ solution to (87) on a neighborhood $\widetilde{\mathcal{V}_{j}}$ of $z_{j}$, then $\widetilde{\Phi}_{j}=\Phi_{j}$ on $\widetilde{\mathcal{V}}_{j} \cap \mathcal{V}_{j}$.

Remark 34. The function $\Phi_{j}$ is actually the Agmon distance to $z_{j}$, see [1, Section 3] for a precise definition of the Agmon distance in a bounded domain. 
Choosing $\varepsilon_{1}$ smaller if necessary, one can assume that there exists $\alpha>0$ such that $B\left(z_{j}, 2 \varepsilon_{1}+\alpha\right) \cap \bar{\Omega} \subset \mathcal{V}_{j}$. The next proposition, which follows from [23, Proposition 4.3.2], gathers all the estimates one needs in the following on the operator $\Delta_{f, h}^{M D,(1)}\left(\mathcal{V}_{j}\right)$.

Proposition 35. Let us assume that $(\mathbf{A 0})$ is satisfied. Then, the operator $\Delta_{f, h}^{M D,(1)}\left(\mathcal{V}_{j}\right)$ is self-adjoint, has compact resolvent and is positive. Moreover:

- There exists $h_{0}>0$ such that for all $h \in\left(0, h_{0}\right)$ :

$$
\operatorname{dim} \operatorname{Ran} \pi_{\left[0, h^{\frac{3}{2}}\right)}\left(\Delta_{f, h}^{M D,(1)}\left(\mathcal{V}_{j}\right)\right)=1 .
$$

- The smallest eigenvalue $\lambda_{h}\left(\mathcal{V}_{j}\right)$ of $\Delta_{f, h}^{M D,(1)}\left(\mathcal{V}_{j}\right)$ is exponentially small: there exist $C>0, c>0$ and $h_{0}>0$ such that for any $h \in\left(0, h_{0}\right)$ :

$$
\lambda_{h}\left(\mathcal{V}_{j}\right) \leq C e^{-\frac{c}{h}}
$$

- Any $L^{2}$-normalized eigenform $w_{j}$ associated with the smallest eigenvalue $\lambda_{h}\left(\mathcal{V}_{j}\right)$ of $\Delta_{f, h}^{M D,(1)}\left(\mathcal{V}_{j}\right)$ satisfies the following Agmon estimates: there exist $C>0, n \in \mathbb{N}$ and $h_{0}>0$ such that for any $h \in\left(0, h_{0}\right)$, it holds:

$$
\left\|e^{\frac{1}{h} \Phi_{j}} w_{j}\right\|_{H^{1}\left(B\left(z_{j}, 2 \varepsilon_{1}\right) \cap \Omega\right)} \leq C h^{-n}
$$

where $\Phi_{j}$ is the solution to (87).

Let us now define the quasi-mode associated with $z_{j} \in U_{1}^{\partial \Omega}$.

Definition 36. Let us assume that (A0) holds. Let $j \in\left\{1, \ldots, \mathrm{m}_{1}^{\partial \Omega}\right\}$ and $z_{j} \in \mathrm{U}_{1}^{\partial \Omega}$. The quasi-mode associated with $z_{j}$ is defined by

$$
\widetilde{\phi}_{j}:=\frac{\theta_{j} w_{j}}{\left\|\theta_{j} w_{j}\right\|_{L^{2}}} \in \Lambda^{1} H_{T}^{1}(\Omega) \cap \Lambda^{1} C^{\infty}(\bar{\Omega})
$$

where $w_{j}$ is a $L^{2}$-normalized eigenform associated with the first eigenvalue $\lambda_{h}\left(\mathcal{V}_{j}\right)$ of $\Delta_{f, h}^{(1), M D}\left(\mathcal{V}_{j}\right)$ and $\theta_{j}$ is

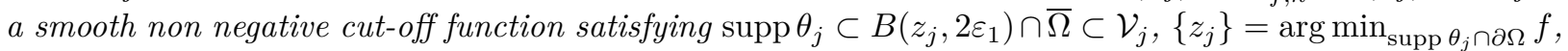
and $\theta_{j}=1$ on $B\left(z_{j}, \varepsilon_{1}\right) \cap \bar{\Omega}$.

Notice again that both $w_{j}$ and $-w_{j}$ can be used to build a quasi-mode: the sign will be chosen in Proposition 38. The fact that $\widetilde{\phi}_{j} \in \Lambda^{1} C^{\infty}(\bar{\Omega})$ follows from standard elliptic regularity results. In addition, for all $j \in\left\{1, \ldots, \mathrm{m}_{1}^{\partial \Omega}\right\}$, using (91) together with the fact that $\inf _{\text {supp }\left(1-\theta_{j}\right) \cap \mathcal{V}_{j}} \Phi_{j}>0$ (see (88)), there exists $c>0$ such that when $h \rightarrow 0$ :

$$
\left\|\left(1-\theta_{j}\right) w_{j}\right\|_{L^{2}\left(\mathcal{V}_{j}\right)}=O\left(e^{-\frac{c}{h}}\right) \text { and thus, }\left\|\theta_{j} w_{j}\right\|_{L^{2}}=1+O\left(e^{-\frac{c}{h}}\right) .
$$

Using Proposition 35 and (93), one deduces the following estimate on the quasi-mode $\widetilde{\phi}_{j}$ introduced in Definition 31.

Corollary 37. Let us assume that (A0) holds. Let $\widetilde{\phi}_{j}$ be the quasi-mode associated with $z_{j} \in U_{1}^{\partial \Omega}(j \in$ $\left.\left\{1, \ldots, \mathrm{m}_{1}^{\partial \Omega}\right\}\right)$, see Definition 36. Then, there exist $C>0, c>0$ and $h_{0}>0$ such that for any $h \in\left(0, h_{0}\right)$ :

$$
\left\|d_{f, h} \widetilde{\phi}_{j}\right\|_{L^{2}}+\left\|d_{f, h}^{*} \widetilde{\phi}_{j}\right\|_{L^{2}} \leq C e^{-\frac{c}{h}} .
$$


Let us now give the WKB approximation estimates for the quasi-mode $\widetilde{\phi}_{j}$ introduced in Definition 36. From [23, Section 4.2], there exists a $C^{\infty}\left(\overline{\mathcal{V}_{j}}\right)$ function $a_{j}(x, h)=\widetilde{a}_{j}(x)+O(h)$ with $a_{j} \equiv \widetilde{a}_{j} \equiv 1$ on $\partial \Omega \cap \overline{\mathcal{V}_{j}}$ such that the 1-form

$$
u_{j, w k b}^{(1)}=d_{f, h}\left(a_{j}(x, h) e^{-\frac{1}{h} \Phi_{j}}\right)=\left(\widetilde{a}_{j} d\left(f-\Phi_{j}\right)+O(h)\right) e^{-\frac{1}{h} \Phi_{j}},
$$

satisfies

$$
\left\{\begin{aligned}
\Delta_{f, h}^{(1)} u_{j, w k b}^{(1)} & =O\left(h^{\infty}\right) e^{-\frac{1}{h} \Phi_{j}} \text { in } \mathcal{V}_{j} \\
\mathbf{t} u_{j, w k b}^{(1)} & =0 \text { on } \partial \Omega \cap \overline{\mathcal{V}_{j}} \\
\mathbf{t} d_{f, h}^{*} u_{j, w k b}^{(1)} & =O\left(h^{\infty}\right) e^{-\frac{1}{h} \Phi_{j}} \text { on } \partial \Omega \cap \overline{\mathcal{V}_{j}}
\end{aligned}\right.
$$

Moreover, one has in the limit $h \rightarrow 0$ (see [23, Section 4.2]):

$$
\left\|\theta_{j} u_{j, w k b}^{(1)}\right\|_{L^{2}}=\frac{\pi^{\frac{d-1}{4}} \sqrt{2 \partial_{n} f\left(z_{j}\right)}}{\left(\left.\operatorname{det} \operatorname{Hess} f\right|_{\partial \Omega}\left(z_{j}\right)\right)^{\frac{1}{4}}} h^{\frac{d+1}{4}}(1+O(h)),
$$

where the remainder term $O(h)$ admits a full asymptotic expansion in $h$. In the following proposition, $w_{j}$ and $u_{j, w k b}^{(1)}$ are compared.

Proposition 38. Let us assume that (A0) holds. Let $w_{j}$ be a $L^{2}$-normalized eigenform associated with the smallest eigenvalue $\lambda_{h}\left(\mathcal{V}_{j}\right)$ of $\Delta_{f, h}^{F D,(1)}\left(\mathcal{V}_{j}\right)\left(j \in\left\{1, \ldots, \mathrm{m}_{1}^{\partial \Omega}\right\}\right)$. Then, there exists $h_{0}>0$ such that for all $h \in\left(0, h_{0}\right)$ one has:

$$
\left\|\theta_{j}\left(w_{j}-c_{j}(h) u_{j, w k b}^{(1)}\right)\right\|_{H^{1}}=O\left(h^{\infty}\right)
$$

where

$$
c_{j}(h)^{-1}=\left\langle w_{j}, \theta_{j} u_{j, w k b}^{(1)}\right\rangle_{L^{2}} .
$$

In addition, up to replacing $w_{j}$ by $-w_{j}$, one can assume that $c_{j}(h) \geq 0$ for $h$ small enough and then, in the limit $h \rightarrow 0$, one has:

$$
c_{j}(h)=\frac{\left(\left.\operatorname{det} \operatorname{Hess} f\right|_{\partial \Omega}\left(z_{j}\right)\right)^{\frac{1}{4}}}{\pi^{\frac{d-1}{4}} \sqrt{2 \partial_{n} f\left(z_{j}\right)}} h^{-\frac{d+1}{4}}(1+O(h)),
$$

where the remainder term $O(h)$ admits a full asymptotic expansion in $h$.

Proposition 38 is proved exactly as Proposition 33.

In conclusion, a family of 1 -forms $\left(\widetilde{\phi}_{j}\right)_{j \in\left\{1, \ldots, \mathrm{m}_{1}^{\bar{\Omega}}\right\}}$ has been constructed in this section. Since (62) guarantees that $\overline{B\left(z, 2 \varepsilon_{1}\right)} \cap \overline{B\left(z^{\prime}, 2 \varepsilon_{1}\right)}=\emptyset$ for all $z \neq z^{\prime} \in \mathrm{U}_{1}^{\bar{\Omega}}$, the family $\left(\widetilde{\phi}_{j}\right)_{j \in\left\{1, \ldots, \mathrm{m}_{1}^{\bar{\Omega}}\right\}}$ is orthonormal in $L^{2}(\Omega)$. From now on, the parameter $\varepsilon_{1}$ is fixed and $\varepsilon>0$ will be successively reduced a finite number of times in the following.

WKB approximation of the quasi-modes $\left(\widetilde{\phi}_{j}\right)_{j \in\left\{1, \ldots, \mathrm{m}_{1}^{\bar{\Omega}}\right\}}$. For upcoming computations, one needs the following definition. 
Definition 39. Let us assume that $(\mathbf{A} 0)$ is satisfied. For all $j \in\left\{1, \ldots, \mathrm{m}_{1}^{\bar{\Omega}}\right\}$, one defines:

$$
\widetilde{\phi}_{j, w k b}:=c_{j}(h) \theta_{j} u_{j, w k b}^{(1)},
$$

where for $j \in\left\{1, \ldots, \mathrm{m}_{1}^{\partial \Omega}\right\}, u_{j, w k b}^{(1)}$ satisfies (96) and $\theta_{j}$ is introduced in Definition 36 and, for $j \in\left\{\mathrm{m}_{1}^{\partial \Omega}+\right.$ $\left.1, \ldots, \mathrm{m}_{1}^{\bar{\Omega}}\right\}, u_{j, w k b}^{(1)}$ satisfies (84) and $\theta_{j}$ is introduced in Definition 31.

From (78), Proposition 33, (93), and Proposition 38 one has the following lemma.

Lemma 40. Let us assume that (A0) is satisfied. For $j \in\left\{1, \ldots, \mathrm{m}_{1}^{\partial \Omega}\right\}$, let $\widetilde{\phi}_{j}$ be as defined in (92), and for $j \in\left\{\mathrm{m}_{1}^{\partial \Omega}+1, \ldots, \mathrm{m}_{1}^{\bar{\Omega}}\right\}$, let $\widetilde{\phi}_{j}$ be as defined in (77). Moreover, for $j \in\left\{1, \ldots, \mathrm{m}_{1}^{\bar{\Omega}}\right\}$, let $\widetilde{\phi}_{j, w k b}$ be as defined in (99). Then, one has:

$$
\left\|\widetilde{\phi}_{j}-\widetilde{\phi}_{j, w k b}\right\|_{H^{1}}=O\left(h^{\infty}\right)
$$

3.2.3. Quasi-modes for $-L_{f, h}^{D,(0)}$ and $-L_{f, h}^{D,(1)}$

Before defining the quasi-modes for $L_{f, h}^{D,(0)}$ and $L_{f, h}^{D,(1)}$, let us label the quasi-modes for $\Delta_{f, h}^{D,(0)}$ and the local minima of $f$ using the lexicographic order.

Definition 41. Let us assume that (A0) is satisfied. Then, the family of critical connected components

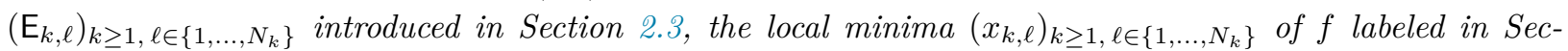
tion 2.3, the family of cut-off functions $\left.\left(\chi_{k, \ell}^{\varepsilon, \varepsilon_{1}}\right)_{k, \ell}\right)_{k \geq 1, \ell \in\left\{1, \ldots, N_{k}\right\}}$ introduced in Definition 26 and the family of quasi-modes $\left(\widetilde{v}_{k, \ell}\right)_{k \geq 1, \ell \in\left\{1, \ldots, N_{k}\right\}}$ introduced in Definition 26 are labeled according to the lexicographic order:

$$
\left(\mathrm{E}_{k}\right)_{k \in\left\{1, \ldots, \mathbf{m}_{0}^{\Omega}\right\}}, \quad\left(\chi_{k}^{\varepsilon, \varepsilon_{1}}\right)_{k \in\left\{1, \ldots, \mathbf{m}_{0}^{\Omega}\right\}},\left(\widetilde{v}_{k}\right)_{k \in\left\{1, \ldots, \mathbf{m}_{0}^{\Omega}\right\}} \text { and }\left(x_{k}\right)_{k \in\left\{1, \ldots, \mathbf{m}_{0}^{\Omega}\right\}} .
$$

Let us recall that the lexicographic order is defined by $\left(k^{\prime}, \ell^{\prime}\right)<(k, l)$ if and only if $k^{\prime}<k$ or if $k^{\prime}=$ $k, \ell^{\prime}<\ell$. From now on, one uses the labeling introduced in Definition 41.

According to (58), the quasi-modes for $L_{f, h}^{D,(0)}$ and $L_{f, h}^{D,(1)}$ are obtained from those constructed previously for $\Delta_{f, h}^{D,(0)}$ and $\Delta_{f, h}^{D,(1)}$ using the unitary transformation $U$ defined in (57).

Definition 42. Let us assume that (A0) is satisfied. Let $\left(\widetilde{v}_{k}\right)_{k \in\left\{1, \ldots, \mathrm{m}_{0}^{\Omega}\right\}}$ be the family of quasi-modes for $\Delta_{f, h}^{D,(0)}$ introduced in Definition 26 and labeled in Definition 41 and let $\left(\widetilde{\phi}_{j}\right)_{j \in\left\{1, \ldots, \mathrm{m}_{1}^{\bar{\Omega}}\right\}}$ be the family of quasi-modes for $\Delta_{f, h}^{D,(1)}$ introduced in Definitions 31 and 36. The family of quasi-modes $\left(\widetilde{u}_{k}\right)_{k \in\left\{1, \ldots, \mathrm{m}_{0}^{\Omega}\right\}}$ for $-L_{f, h}^{D,(0)}$ and the family of quasi-modes $\left(\widetilde{\psi}_{j}\right)_{j \in\left\{1, \ldots, \mathrm{m}_{1}^{\bar{\Omega}}\right\}}$ for $-L_{f, h}^{D,(1)}$ are defined by: for $k \in\left\{1, \ldots, \mathrm{m}_{0}^{\Omega}\right\}$, and for $j \in\left\{1, \ldots, \mathrm{m}_{1}^{\bar{\Omega}}\right\}$ :

$$
\widetilde{u}_{k}:=e^{\frac{1}{h} f} \widetilde{v}_{k} \in \Lambda^{0} H_{w, T}^{1}(\Omega) \quad \text { and } \quad \widetilde{\psi}_{j}:=e^{\frac{1}{h} f} \widetilde{\phi}_{j} \in \Lambda^{1} H_{w, T}^{1}(\Omega) .
$$

Notice that, according to (65) and (100), for all $k \in\left\{1, \ldots, \mathrm{m}_{0}^{\Omega}\right\}, \widetilde{u}_{k} \in C_{c}^{\infty}(\Omega)$, and according to $(77)$ and (92), for all $j \in\left\{1, \ldots, \mathrm{m}_{1}^{\bar{\Omega}}\right\}, \widetilde{\psi}_{j} \in \Lambda^{1} C^{\infty}(\bar{\Omega})$.

3.3. Bases of $\operatorname{Ran} \pi_{h}^{(0)}$ and $\operatorname{Ran} \pi_{h}^{(1)}$

Let us recall, that from $(60), \pi_{h}^{(0)}=\pi_{\left[0, \frac{\sqrt{h}}{2}\right)}\left(-L_{f, h}^{D,(0)}\right)$ and $\pi_{h}^{(1)}=\pi_{\left[0, \frac{\sqrt{h}}{2}\right)}\left(-L_{f, h}^{D,(1)}\right)$. In this section, one proves that the spectral projections of the quasi-modes introduced in Definition 42 form bases of Ran $\pi_{h}^{(0)}$ 
and $\operatorname{Ran} \pi_{h}^{(1)}$. In the following, the finite dimensional spaces $\operatorname{Ran} \pi_{h}^{(0)}$ and $\operatorname{Ran} \pi_{h}^{(1)}$ are endowed with the scalar product $\langle\cdot, \cdot\rangle_{L_{w}^{2}}$.

Proposition 43. Let us assume that $(\mathbf{A} 0)$ holds. Let $\left(\widetilde{u}_{k}\right)_{k \in\left\{1, \ldots, \mathrm{m}_{0}^{\Omega}\right\}}$ be the family of quasi-modes for $-L_{f, h}^{D,(0)}$ and let $\left(\widetilde{\psi}_{j}\right)_{j \in\left\{1, \ldots, \mathrm{m}_{1}^{\bar{\Omega}}\right\}}$ be the family of quasi-modes for $-L_{f, h}^{D,(1)}$ introduced in Definition 42 . Then,

1. For all $k \in\left\{1, \ldots, \mathrm{m}_{0}^{\Omega}\right\}$ and $j \in\left\{1, \ldots, \mathrm{m}_{1}^{\bar{\Omega}}\right\}, \widetilde{u}_{k} \in \Lambda^{0} H_{w, T}^{1}(\Omega), \widetilde{\psi}_{j} \in \Lambda^{1} H_{w, T}^{1}(\Omega)$ and

$$
\left\|\widetilde{u}_{k}\right\|_{L_{w}^{2}}=\left\|\widetilde{\psi}_{j}\right\|_{L_{w}^{2}}=1 \text { and } \forall i \in\left\{1, \ldots, \mathrm{m}_{1}^{\bar{\Omega}}\right\} \backslash\{j\},\left\langle\widetilde{\psi}_{j}, \widetilde{\psi}_{i}\right\rangle_{L_{w}^{2}}=0
$$

2. a) For any $\delta>0$, one can choose the parameter $\varepsilon$ in (65) (see also (100)) small enough such that for all $k \in\left\{1, \ldots, \mathrm{m}_{0}^{\Omega}\right\}$, in the limit $h \rightarrow 0:$

$$
\left\|\left(1-\pi_{h}^{(0)}\right) \widetilde{u}_{k}\right\|_{L_{w}^{2}}^{2} \leq h^{\frac{1}{2}}\left\|\nabla \widetilde{u}_{k}\right\|_{L_{w}^{2}}^{2}=O\left(e^{-\frac{2}{h}\left(f\left(\mathbf{j}\left(x_{k}\right)\right)-f\left(x_{k}\right)-\delta\right)}\right) .
$$

In particular, choosing the parameter $\varepsilon>0$ small enough in (65), there exists $c>0$ such that in the limit $h \rightarrow 0$ :

$$
\pi_{h}^{(0)} \widetilde{u}_{k}=\widetilde{u}_{k}+O\left(e^{-\frac{c}{h}}\right) \text { in } L_{w}^{2}(\Omega)
$$

b) There exist $c>0$ such that for all $j \in\left\{1, \ldots, \mathrm{m}_{1}^{\bar{\Omega}}\right\}$, one has in the limit $h \rightarrow 0$ :

$$
\left\|\left(1-\pi_{h}^{(1)}\right) \widetilde{\psi}_{j}\right\|_{H_{w}^{1}}^{2}=O\left(e^{-\frac{c}{h}}\right) .
$$

3. a) The family $\left(\widetilde{u}_{k}\right)_{k=1, \ldots, \mathrm{m}_{0}^{\Omega}}$ is uniformly linearly independent (for the $L_{w}^{2}$-scalar product) for all $h$ sufficiently small (as defined in Lemma 28).

b) For all $(i, j) \in\left\{1, \ldots, \mathrm{m}_{1}^{\bar{\Omega}}\right\}^{2}$,

$$
\left\langle\pi_{h}^{(1)} \widetilde{\psi}_{i}, \pi_{h}^{(1)} \widetilde{\psi}_{j}\right\rangle_{L_{w}^{2}}=\delta_{i, j}+O\left(e^{-\frac{c}{h}}\right)
$$

In particular, there exists $h_{0}>0$ such that for all $h \in\left(0, h_{0}\right)$ :

$$
\operatorname{Ran} \pi_{h}^{(0)}=\operatorname{Span}\left(\pi_{h}^{(0)} \widetilde{u}_{k}, k=1, \ldots, \mathrm{m}_{0}^{\Omega}\right) \text { and } \operatorname{Ran} \pi_{h}^{(1)}=\operatorname{Span}\left(\pi_{h}^{(1)} \widetilde{\psi}_{i}, i=1, \ldots, \mathrm{m}_{1}^{\bar{\Omega}}\right)
$$

Proof. The proof of Proposition 43 is divided into two steps.

Step 1: Proofs of items 1 and 2. The first item is immediate according to the definition of the families $\left(\widetilde{u}_{k}\right)_{k=1, \ldots, \mathrm{m}_{0}^{\Omega}}$ and $\left(\widetilde{\psi}_{i}\right)_{i=1, \ldots, \mathrm{m}_{1}^{\bar{\Omega}}}$ introduced in Definition 42 .

The first upper bound appearing in $2 \mathrm{a}$ is a direct consequence of Lemma 25 applied to $A=-L_{f, h}^{D,(0)}$ whose associated quadratic form is given by $\frac{h}{2}\langle\nabla \cdot \nabla \cdot\rangle_{L_{w}^{2}}$ on $H_{w, T}^{1}(\Omega)$. The second upper bound in 2 a follows from Laplace's methods and from the properties of the cut-off functions used to define the quasi-modes $\widetilde{u}_{k}$ (see Definition 42 and Lemma 27). Indeed, it is just a rewriting of (71) using Definition 42 and the labeling introduced in Definition 41.

Let us now deal with $2 \mathrm{~b}$. First, Lemma 25 together with (79) and (94) implies the existence of some $c>0$ such that for all $i \in\left\{1, \ldots, \mathrm{m}_{1}^{\bar{\Omega}}\right\}$ and $h$ small enough,

$$
\left\|\left(1-\pi_{\left[0, h^{\frac{3}{2}}\right)}\left(\Delta_{f, h}^{D,(1)}\right)\right) \widetilde{\phi}_{i}\right\|_{L^{2}}=O\left(e^{-\frac{c}{h}}\right) .
$$


Consequently, using again (79) and (94), and owing to the following relations on $\Lambda^{1} H_{T}^{1}(\Omega): d_{f, h}(1-$ $\left.\pi_{\left[0, h \frac{3}{2}\right)}\left(\Delta_{f, h}^{D,(1)}\right)\right)=\left(1-\pi_{\left[0, h^{\frac{3}{2}}\right)}\left(\Delta_{f, h}^{D,(2)}\right)\right) d_{f, h}, d_{f, h}^{*}\left(1-\pi_{\left[0, h \frac{3}{2}\right)}\left(\Delta_{f, h}^{D,(1)}\right)\right)=\left(1-\pi_{\left[0, h^{\frac{3}{2}}\right)}\left(\Delta_{f, h}^{D,(0)}\right)\right) d_{f, h}^{*}$, $d_{f, h}^{*}=h d^{*}+\mathbf{i}_{\nabla f}$, and $d_{f, h}=h d+\nabla f \wedge$, one obtains the existence of $c>0$ such that in the limit $h \rightarrow 0$ :

$$
\left\|d\left(1-\pi_{\left[0, h^{\frac{3}{2}}\right)}\left(\Delta_{f, h}^{D,(1)}\right)\right) \widetilde{\phi}_{i}\right\|_{L^{2}}+\left\|d^{*}\left(1-\pi_{\left[0, h^{\frac{3}{2}}\right)}\left(\Delta_{f, h}^{D,(1)}\right)\right) \widetilde{\phi}_{i}\right\|_{L^{2}}=O\left(e^{-\frac{c}{h}}\right) .
$$

Since $\widetilde{\phi}_{i} \in \Lambda^{1} H_{T}^{1}(\Omega)$, the estimates (101) and (102) then lead, owing to Gaffney's inequality (see [33, Corollary 2.1.6]), to

$$
\left\|\left(1-\pi_{\left[0, h^{\frac{3}{2}}\right)}\left(\Delta_{f, h}^{D,(1)}\right)\right) \widetilde{\phi}_{i}\right\|_{H^{1}}=O\left(e^{-\frac{c}{h}}\right)
$$

Therefore, we deduce from the relation $\|u\|_{H_{w}^{1}} \leq \frac{C}{h}\left\|u e^{-\frac{1}{h} f}\right\|_{H^{1}}$, valid for all $u \in \Lambda^{p} H^{1}(\Omega)$ and $h>0$, and from $\pi_{\left[0, h^{\frac{3}{2}}\right)}\left(\Delta_{f, h}^{D,(1)}\right)=e^{-\frac{1}{h} f} \pi_{h}^{(1)} e^{\frac{1}{h} f}$, resulting from (58) and (60), that there exists $c>0$ such that for all $i \in\left\{1, \ldots, \mathrm{m}_{1}^{\bar{\Omega}}\right\}$ and $h$ small enough,

$$
\left\|\left(1-\pi_{h}^{(1)}\right) \widetilde{\psi}_{i}\right\|_{H_{w}^{1}} \leq \frac{C}{h}\left\|\left(1-\pi_{\left[0, h^{\frac{3}{2}}\right)}\left(\Delta_{f, h}^{D,(1)}\right)\right) \widetilde{\phi}_{i}\right\|_{H^{1}}=O\left(e^{-\frac{c}{h}}\right) .
$$

This ends the proof of $2 \mathrm{~b}$.

Step 2: Proof of item 3. The fact that the family $\left(\widetilde{u}_{k}\right)_{k=1, \ldots, \mathbf{m}_{0}^{\Omega}}$ is uniformly linearly independent is a consequence of Lemma 28 together with (100). Item $3 \mathrm{~b}$ follows from items 1 and $2 \mathrm{~b}$ together with the relation

$$
\left\langle\pi_{h}^{(\ell)} f, \pi_{h}^{(\ell)} g\right\rangle_{L_{w}^{2}}=-\left\langle\left(\pi_{h}^{(\ell)}-1\right) f,\left(\pi_{h}^{(\ell)}-1\right) g\right\rangle_{L_{w}^{2}}+\langle f, g\rangle_{L_{w}^{2}}
$$

holding for $f, g$ in $\Lambda^{\ell} L_{w}^{2}(\Omega)$ and $\ell \in\{0,1\}$. Finally, the fact that for $h$ small enough, $\operatorname{Ran} \pi_{h}^{(0)}=$ $\operatorname{Span}\left(\pi_{h}^{(0)} \widetilde{u}_{k}, \quad k=1, \ldots, \mathrm{m}_{0}^{\Omega}\right)$ and $\operatorname{Ran} \pi_{h}^{(1)}=\operatorname{Span}\left(\pi_{h}^{(1)} \widetilde{\psi}_{i}, \quad i=1, \ldots, \mathrm{m}_{1}^{\bar{\Omega}}\right)$ are consequences of items 2a, $3 \mathrm{a}$ and $3 \mathrm{~b}$ together with Lemma 23.

\section{On the smallest eigenvalue of $-L_{f, h}^{D,(0)}$}

This section is dedicated to the proof of the following theorem.

Theorem 2. Assume that the assumptions $(\mathbf{A} 0)$ and $(\mathbf{A} 1 \mathbf{j})$ are satisfied. Let $\lambda_{h}$ be the principal eigenvalue of $-L_{f, h}^{D,(0)}$ (see (8)). Then, denoting by $\lambda_{2, h}$ the second smallest eigenvalue of $-L_{f, h}^{D,(0)}$, there exists $c>0$ such that in the limit $h \rightarrow 0$ :

$$
\lambda_{h}=\lambda_{2, h} O\left(e^{-\frac{c}{h}}\right) .
$$

Moreover, when (A2j) is satisfied, one has in the limit $h \rightarrow 0$ :

$$
\lambda_{h}=\frac{\sum_{z \in \partial \mathrm{C}_{1} \cap \partial \Omega} \partial_{n} f(z)\left(\left.\operatorname{det} \operatorname{Hess} f\right|_{\partial \Omega}(z)\right)^{-\frac{1}{2}}}{\sqrt{\pi h} \sum_{x \in \arg \min _{\mathrm{C}_{1}} f}(\operatorname{det} \operatorname{Hess} f(x))^{-\frac{1}{2}}} e^{-\frac{2}{h}\left(f\left(\mathbf{j}\left(x_{1}\right)\right)-f\left(x_{1}\right)\right)}(1+O(\sqrt{h}))
$$

where we recall that $x_{1} \in \arg \min _{\mathrm{C}_{1}} f$. Finally, when $(\mathbf{A} 4 \mathbf{j})$ holds, the remainder term $O(\sqrt{h})$ in $(105)$ is actually of order $O(h)$ and admits a full asymptotic expansion in $h$. 
Remark 44. Without the assumption (A4j), we are not able to prove an asymptotic expansion in $\sqrt{h}$ of the remainder term $O(\sqrt{h})$ in (105) except in some specific cases, see Theorem 3 below or [27, Proposition C.40].

Let us mention that sharp asymptotic estimates when $h \rightarrow 0$ of the principal eigenvalue of $-L_{f, h}^{D,(0)}$ have been obtained in $[23,17,1]$ in the Dirichlet case and in [37] in the Neumann case. However, these results do not apply under the assumptions considered in Theorem 2 . When $\Omega=\mathbb{R}^{d}$ or when $\Omega$ is a compact Riemannian manifold, sharp asymptotic estimates of the second smallest eigenvalue of $-L_{f, h}^{(0)}$ have been obtained in $[30,31,38,39,28,40,41]$.

The analysis led in this section will also provide lower and upper bounds for the $\mathrm{m}_{0}^{\Omega}$ small eigenvalues of $-L_{f, h}^{D,(0)}$ (and not only $\lambda_{h}$ ) under the sole assumption (A0). This is the purpose of Theorem 4 below.

Remark 45. Combining Theorem 2 and Proposition 6, under the assumptions $(\mathbf{A} \mathbf{0}),(\mathbf{A} 1 \mathbf{j})$ and $(\mathbf{A} 2 \mathbf{j})$, one obtains that in the limit $h \rightarrow 0$ :

$$
\mathbb{E}_{\nu_{h}}\left[\tau_{\Omega}\right]=\frac{1}{\lambda_{h}}=\frac{\sqrt{\pi h} \sum_{x \in \arg \min _{\mathrm{C}_{1}} f}(\operatorname{det} \operatorname{Hess} f(x))^{-\frac{1}{2}}}{\sum_{z \in \partial \mathrm{C}_{1} \cap \partial \Omega} \partial_{n} f(z)\left(\left.\operatorname{det} \operatorname{Hess} f\right|_{\partial \Omega}(z)\right)^{-\frac{1}{2}}} e^{\frac{2}{h}\left(f\left(\mathbf{j}\left(x_{1}\right)\right)-f\left(x_{1}\right)\right)}(1+O(\sqrt{h})) .
$$

In some specific cases, one can drop the assumption (A2j) in Theorem 2 and still obtain a sharp asymptotic equivalent of $\lambda_{h}$ when $h \rightarrow 0$. Indeed, the arguments in the proof of Theorem 2 also give the following result:

Theorem 3. Assume that the assumptions $(\mathbf{A 0})$ and $(\mathbf{A} 1 \mathbf{j})$ are satisfied. Assume moreover that for all $j \in$ $\left\{2, \ldots, \mathrm{N}_{1}\right\}, \partial \mathrm{C}_{1} \cap \partial \mathrm{C}_{j}=\emptyset$ (this last assumption is for instance satisfied when $\mathrm{N}_{1}=1$ ). Let us define,

$$
\begin{gathered}
\text { when } \partial \mathrm{C}_{1} \cap \partial \Omega \neq \emptyset, a_{1}:=\frac{\sum_{z \in \partial \mathrm{C}_{1} \cap \partial \Omega} \partial_{n} f(z)\left(\left.\operatorname{det} \operatorname{Hess} f\right|_{\partial \Omega}(z)\right)^{-\frac{1}{2}}}{\sqrt{\pi} \sum_{x \in \arg \min _{\mathrm{C}_{1}} f}(\operatorname{det} \operatorname{Hess} f(x))^{-\frac{1}{2}}}, \text { else, } a_{1}:=0, \\
\text { when } \partial \mathrm{C}_{1} \cap \mathrm{U}_{1}^{\mathrm{ssp}} \cap \Omega \neq \emptyset, a_{2}:=\frac{1}{2 \pi} \frac{\sum_{z \in \partial \mathrm{C}_{1} \cap U_{1}^{\text {ssp } \cap \Omega}}\left|\lambda_{-}(z)\right|(\operatorname{det} \operatorname{Hess} f(z))^{-\frac{1}{2}}}{\sum_{x \in \arg \min _{\mathrm{C}_{1}} f}(\operatorname{det} \operatorname{Hess} f(x))^{-\frac{1}{2}}}, \text { else, } a_{2}:=0,
\end{gathered}
$$

where $\lambda_{-}(z)$ is the negative eigenvalue of Hess $f(z)$ (notice that $a_{1}$ and $a_{2}$ cannot be both equal to 0 since from Proposition 15, $\left.\partial \mathrm{C}_{1} \cap \mathrm{U}_{1}^{\text {ssp }} \neq \emptyset\right)$. Then, one has when $h \rightarrow 0$ :

$$
\lambda_{h}=\left[\frac{a_{1}}{\sqrt{h}}(1+O(h))+a_{2}(1+O(h))\right] e^{-\frac{2}{h}\left(f\left(\mathbf{j}\left(x_{1}\right)\right)-f\left(x_{1}\right)\right)},
$$

where the two remainder terms $O(h)$ admit a full asymptotic expansion in $h$.

This section is organized as follows. In Section 4.1, one gives the quasi-modal estimates which are used to prove Theorems 2 and 3. Section 4.2 is then dedicated to the proof of Theorems 2 and 3.

\subsection{Estimates of interactions between quasi-modes}

The main result of this section is Proposition 47 which gives the quasi-modal estimates in $L_{w}^{2}(\Omega)$ needed to prove Theorem 2. This section is divided into two parts. In Section 4.1.1, one gives the asymptotic estimates 
of the boundary terms $\left(\int_{\Sigma} F \widetilde{\psi}_{j} \cdot n e^{-\frac{2}{h} f}\right)_{j \in\left\{1, \ldots, \mathrm{m}_{1}^{\bar{\Omega}}\right\}}$, which are then used in the proof of Proposition 47. In Section 4.1.2, one states and proves Proposition 47.

For future references, let us define the constants: for all $j \in\left\{1, \ldots, \mathrm{m}_{1}^{\bar{\Omega}}\right\}$,

$$
B_{j}= \begin{cases}\pi^{\frac{d-1}{4}} \sqrt{2 \partial_{n} f\left(z_{j}\right)}\left(\left.\operatorname{det} \operatorname{Hess} f\right|_{\partial \Omega}\left(z_{j}\right)\right)^{-1 / 4} & \text { if } z_{j} \in \partial \Omega \\ \pi^{\frac{d-2}{4}} \sqrt{\left|\lambda_{-}\left(z_{j}\right)\right|}\left|\operatorname{det} \operatorname{Hess} f\left(z_{j}\right)\right|^{-1 / 4} & \text { if } z_{j} \in \Omega\end{cases}
$$

where $\lambda_{-}\left(z_{j}\right)$ is the negative eigenvalue of Hess $f\left(z_{j}\right)$.

\subsubsection{Asymptotic estimates of boundary terms for $\left(\widetilde{\psi}_{j}\right)_{j \in\left\{1, \ldots, \mathrm{m}_{1}^{\bar{\Omega}}\right\}}$}

The following boundary estimates will be used several times in the sequel.

Proposition 46. Let us assume that (A0) is satisfied. Let us consider $j \in\left\{1, \ldots, \mathrm{m}_{1}^{\bar{\Omega}}\right\}$, an open set $\Sigma$ of $\partial \Omega$, and $F \in L^{\infty}(\partial \Omega, \mathbb{R})$. Then, there exists $c>0$ such that one has in the limit $h \rightarrow 0$ :

$$
\int_{\Sigma} F \widetilde{\psi}_{j} \cdot n e^{-\frac{2}{h} f}= \begin{cases}0 & \text { if } j \in\left\{\mathrm{m}_{1}^{\partial \Omega}+1, \ldots, \mathrm{m}_{1}^{\bar{\Omega}}\right\}, \\ O\left(e^{-\frac{1}{h}\left(f\left(z_{j}\right)+c\right)}\right) & \text { if } j \in\left\{1, \ldots, \mathrm{m}_{1}^{\partial \Omega}\right\} \text { and } z_{j} \notin \Sigma, \\ O\left(h^{\frac{d-3}{4}} e^{-\frac{1}{h} f\left(z_{j}\right)}\right) & \text { if } j \in\left\{1, \ldots, \mathrm{m}_{1}^{\partial \Omega}\right\} \text { and } z_{j} \in \Sigma,\end{cases}
$$

where $\widetilde{\psi}_{j}$ is introduced in (100) and $\mathrm{m}_{1}^{\partial \Omega}$ is defined in (18). Moreover, when $j \in\left\{1, \ldots, \mathrm{m}_{1}^{\partial \Omega}\right\}, z_{j} \in \Sigma$, and $F$ is $C^{\infty}$ in a neighborhood of $z_{j}$, it holds

$$
\int_{\Sigma} F \widetilde{\psi}_{j} \cdot n e^{-\frac{2}{h} f}=h^{\frac{d-3}{4}} e^{-\frac{1}{h} f\left(z_{j}\right)}\left(B_{j} F\left(z_{j}\right)+O(h)\right),
$$

where the remainder term $O(h)$ admits a full asymptotic expansion in $h$ and $B_{j}$ is defined by (106).

Proof. Let $F \in L^{\infty}(\partial \Omega, \mathbb{R})$. From $(100)$ and $(77)$, the quasi-mode $\widetilde{\psi}_{j}$ is supported in $\Omega$ for $j \in\left\{\mathrm{m}_{1}^{\partial \Omega}+\right.$ $\left.1, \ldots, \mathrm{m}_{1}^{\bar{\Omega}}\right\}$ and thus:

$$
\forall j \in\left\{\mathrm{m}_{1}^{\partial \Omega}+1, \ldots, \mathrm{m}_{1}^{\bar{\Omega}}\right\}, \int_{\Sigma} F \widetilde{\psi}_{j} \cdot n e^{-\frac{2}{h} f}=0 .
$$

Let us now consider the case $j \in\left\{1, \ldots, \mathrm{m}_{1}^{\partial \Omega}\right\}$. Notice that one has for all $h$ small enough, from the trace theorem, (100), (92), and (91),

$$
\begin{aligned}
\int_{\Sigma} F \widetilde{\psi}_{j} \cdot n e^{-\frac{2}{h} f} & =\int_{\operatorname{supp} \theta_{j} \cap \Sigma} F \widetilde{\phi}_{j} \cdot n e^{-\frac{1}{h} f}=O\left(\left\|\widetilde{\phi}_{j}\right\|_{H^{1}}\right)\left(\int_{\operatorname{supp} \theta_{j} \cap \Sigma} e^{-\frac{2}{h} f}\right)^{\frac{1}{2}} \\
& =O\left(h^{-p}\right)\left(\int_{\operatorname{supp} \theta_{j} \cap \Sigma} e^{-\frac{2}{h} f}\right)^{\frac{1}{2}}
\end{aligned}
$$

where $p$ is independent of $h$. Therefore, since $z_{j}$ is the only minimum of $f$ on $\operatorname{supp} \theta_{j} \cap \partial \Omega$, if $z_{j} \notin \bar{\Sigma}$, one has in the limit $h \rightarrow 0$ :

$$
\int_{\Sigma} F \widetilde{\psi}_{j} \cdot n e^{-\frac{2}{h} f}=O\left(e^{-\frac{1}{h}\left(f\left(z_{j}\right)+c\right)}\right)
$$


for some $c>0$ independent of $h$.

Let us now consider the case $j \in\left\{1, \ldots, \mathrm{m}_{1}^{\partial \Omega}\right\}$ and $z_{j} \in \Sigma$. One has:

$$
\begin{aligned}
\int_{\Sigma} F \widetilde{\psi}_{j} \cdot n e^{-\frac{2}{h} f} & =\int_{\Sigma} F \widetilde{\phi}_{j} \cdot n e^{-\frac{1}{h} f} \\
& =\int_{\Sigma} F \widetilde{\phi}_{j, w k b} \cdot n e^{-\frac{1}{h} f}+\int_{\Sigma} F\left(\widetilde{\phi}_{j}-\widetilde{\phi}_{j, w k b}\right) \cdot n e^{-\frac{1}{h} f},
\end{aligned}
$$

where $\widetilde{\phi}_{j, w k b}=c_{j}(h) \theta_{j} u_{j, w k b}^{(1)}$ is defined in (99). From (95), let us recall that in the limit $h \rightarrow 0, u_{j, w k b}^{(1)}=$ $e^{-\frac{1}{h} \Phi_{j}}\left(\widetilde{a}_{j} d\left(f-\Phi_{j}\right)+O(h)\right)$ on $\operatorname{supp} \theta_{j}$ with $\widetilde{a}_{j}=1$ on $\partial \Omega \cap \operatorname{supp} \theta_{j}$. Thus on $\partial \Omega \cap \operatorname{supp} \theta_{j}$, using also (87), $n \cdot u_{j, w k b}^{(1)}=e^{-\frac{1}{h} \Phi_{j}} \partial_{n}\left(f-\Phi_{j}\right)(1+O(h))=2 \partial_{n} f e^{-\frac{1}{h}\left(f-f\left(z_{j}\right)\right)}(1+O(h))$. Thus, the term $\int_{\Sigma} F \widetilde{\phi}_{j, w k b} \cdot n e^{-\frac{1}{h} f}$ appearing in the right-hand side of (110) satisfies in the limit $h \rightarrow 0$ :

$$
\begin{aligned}
\int_{\Sigma} F \widetilde{\phi}_{j, w k b} \cdot n e^{-\frac{1}{h} f} & =c_{j}(h) \int_{\Sigma \cap \operatorname{supp} \theta_{j}} F \theta_{j} u_{j, w k b}^{(1)} \cdot n e^{-\frac{1}{h} f} \\
& =c_{j}(h) \int_{\Sigma \cap \operatorname{supp} \theta_{j}} 2 \partial_{n} f F \theta_{j} e^{-\frac{1}{h}\left(2 f-f\left(z_{j}\right)\right)}(1+O(h)) \\
& =O\left(c_{j}(h) \int_{\partial \Omega \cap \operatorname{supp} \theta_{j}} 2 \partial_{n} f \theta_{j} e^{-\frac{1}{h}\left(2 f-f\left(z_{j}\right)\right)}\right) \\
& =O\left(c_{j}(h) h^{\frac{d-1}{2}} e^{-\frac{1}{h} f\left(z_{j}\right)}\right)=O\left(h^{\frac{d-3}{4}} e^{-\frac{1}{h} f\left(z_{j}\right)}\right)
\end{aligned}
$$

where the last line follows from $\left\{z_{j}\right\}=\arg \min _{\partial \Omega \cap \operatorname{supp} \theta_{j}} f, \theta_{j}\left(z_{j}\right)=1$, Laplace's method, and $c_{j}(h)=$ $O\left(h^{-\frac{d+1}{4}}\right)$ according to (98). When $z_{j} \in \Sigma$ and $F$ is $C^{\infty}$ in a neighborhood of $z_{j}$, the same arguments applied to (111) yield, in the limit $h \rightarrow 0$ :

$$
\int_{\Sigma} F \widetilde{\phi}_{j, w k b} \cdot n e^{-\frac{1}{h} f}=c_{j}(h) \frac{2 \partial_{n} f\left(z_{j}\right) \pi^{\frac{d-1}{2}}}{\sqrt{\left.\operatorname{det} \operatorname{Hess} f\right|_{\partial \Omega}\left(z_{j}\right)}} h^{\frac{d-1}{2}} e^{-\frac{1}{h} f\left(z_{j}\right)}\left(F\left(z_{j}\right)+O(h)\right),
$$

where the remainder term $O(h)$ admits a full asymptotic expansion in $h$ (which follows from Laplace's method). Besides, from the trace theorem and Lemma 40, the second term in the right-hand side of (110) satisfies in the limit $h \rightarrow 0$ :

$$
\int_{\Sigma} F\left(\widetilde{\phi}_{j}-\widetilde{\phi}_{j, w k b}\right) \cdot n e^{-\frac{1}{h} f}=O\left(h^{\infty}\right) e^{-\frac{1}{h} f\left(z_{j}\right)} .
$$

The first part of Proposition 46 then results from (107)-(110), (112), and (114), and its second part from (110), (113)-(114), and from the asymptotic estimate of $c_{j}(h)$ given in (98) which yields, when $h \rightarrow 0$

$$
\int_{\Sigma} F \widetilde{\psi}_{j} \cdot n e^{-\frac{2}{h} f}=\frac{\pi^{\frac{d-1}{4}} \sqrt{2 \partial_{n} f\left(z_{j}\right)}}{\left(\left.\operatorname{det} \operatorname{Hess} f\right|_{\partial \Omega}\left(z_{j}\right)\right)^{1 / 4}} h^{\frac{d-3}{4}} e^{-\frac{1}{h} f\left(z_{j}\right)}\left(F\left(z_{j}\right)+O(h)\right) .
$$

This concludes the proof of Proposition 46. 


\subsubsection{Quasi-modal estimates in $L_{w}^{2}(\Omega)$}

We are now in position to prove Proposition 47 which will be crucial to prove Theorem 2. This proposition allows indeed to study accurately the singular values of the restricted differential $\nabla: \operatorname{Ran} \pi_{h}^{(0)} \rightarrow \operatorname{Ran} \pi_{h}^{(1)}$ and hence the low lying spectrum of $-L_{f, h}^{D,(0)}$. The square of its smallest singular value is indeed $\frac{2}{h} \lambda_{h}$, where $\lambda_{h}$ is the principal eigenvalue of $-L_{f, h}^{D,(0)}$ (see (8) and Proposition 4).

Proposition 47. Let us assume that (A0) holds. Let $\left(\widetilde{u}_{k}\right)_{k \in\left\{1, \ldots, \mathrm{m}_{0}^{\Omega}\right\}}$ (resp. $\left.\left(\widetilde{\psi}_{j}\right)_{j \in\left\{1, \ldots, \mathrm{m}_{1}^{\bar{\Omega}}\right\}}\right)$ be the family of quasi-modes for $-L_{f, h}^{D,(0)}$ (resp. $\left.-L_{f, h}^{D,(1)}\right)$ introduced in Definition 42. Then, there exists $\varepsilon_{0}>0$ such that for all $\varepsilon \in\left(0, \varepsilon_{0}\right)$, for all $k \in\left\{1, \ldots, \mathrm{m}_{0}^{\Omega}\right\}$ and $j \in\left\{1, \ldots, \mathrm{m}_{1}^{\bar{\Omega}}\right\}$, there exists $\varepsilon_{j, k} \in\{-1,1\}$ independent of $h$ such that in the limit $h \rightarrow 0$,

$$
\left\langle\nabla \widetilde{u}_{k}, \widetilde{\psi}_{j}\right\rangle_{L_{w}^{2}}= \begin{cases}\varepsilon_{j, k} C_{j, k} h^{p_{j, k}} e^{-\frac{1}{h}\left(f\left(\mathbf{j}\left(x_{k}\right)\right)-f\left(x_{k}\right)\right)}(1+O(h)) & \text { if } z_{j} \in \mathbf{j}\left(x_{k}\right), \\ 0 & \text { if } z_{j} \notin \mathbf{j}\left(x_{k}\right),\end{cases}
$$

where all the remainder term $O(h)$ admits a full asymptotic expansions in $h$,

$$
p_{j, k}= \begin{cases}-\frac{1}{2} & \text { if } z_{j} \in \mathbf{j}\left(x_{k}\right) \cap \Omega \\ -\frac{3}{4} & \text { if } z_{j} \in \mathbf{j}\left(x_{k}\right) \cap \partial \Omega\end{cases}
$$

and

$$
C_{j, k}=\frac{B_{j} \pi^{-\frac{d}{4}}}{\left(\sum_{x \in \arg \min _{\mathrm{E}_{k}} f}(\operatorname{det} \operatorname{Hess} f(x))^{-\frac{1}{2}}\right)^{1 / 2}},
$$

where the constant $B_{j}$ is defined in (106), and $\left(\mathrm{E}_{k}\right)_{k \in\left\{1, \ldots, \mathrm{m}_{0}^{\Omega}\right\}}$ is defined in Section 2.3 and labeled in Definition 41. Finally, if $z_{j} \in \mathbf{j}\left(x_{k}\right) \cap \partial \Omega$ (and thus, it holds necessarily $k \in\left\{1, \ldots, \mathrm{N}_{1}\right\}$, see (46) and (34) for the definition of $\mathrm{N}_{1}$ ), one has $\varepsilon_{j, k}=-1$.

Proof. The proof of Proposition 47 is divided into three steps.

Step 1. Let $k \in\left\{1, \ldots, \mathrm{m}_{0}^{\Omega}\right\}$ and $j \in\left\{1, \ldots, \mathrm{m}_{1}^{\bar{\Omega}}\right\}$. Let us consider the case $z_{j} \notin \mathbf{j}\left(x_{k}\right)$. According to Definitions 31,36 and 42 , one has that for all $j \in\left\{1, \ldots, \mathrm{m}_{1}^{\bar{\Omega}}\right\}$, the quasi-mode $\widetilde{\psi}_{j}$ is supported in $B\left(z_{j}, 2 \varepsilon_{1}\right) \cap \bar{\Omega}$. Moreover, from (65), (69), and Definition 42, one has supp $\nabla \chi_{k}^{\varepsilon, \varepsilon_{1}} \cap B\left(z_{j}, 2 \varepsilon_{1}\right)=\emptyset$ and thus $\left\langle\nabla \widetilde{u}_{k}, \widetilde{\psi}_{j}\right\rangle_{L_{w}^{2}}=0$.

Step 2. Let us now deal with the computation of the terms $\left\langle\nabla \widetilde{u}_{k}, \widetilde{\psi}_{j}\right\rangle_{L_{w}^{2}}$ for $k \in\left\{1, \ldots, \mathrm{m}_{0}^{\Omega}\right\}$ and $j \in$ $\left\{1, \ldots, \mathrm{m}_{1}^{\bar{\Omega}}\right\}$ such that $z_{j} \in \mathbf{j}\left(x_{k}\right) \cap \Omega$. In this case, we follow the proof of [31, Proposition 6.4], the only

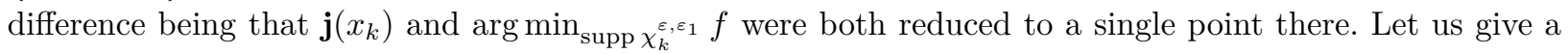
proof for the sake of completeness. One has:

$$
\left\langle\nabla \widetilde{u}_{k}, \widetilde{\psi}_{j}\right\rangle_{L_{w}^{2}}=\int_{\Omega} \nabla \widetilde{u}_{k} \cdot \widetilde{\phi}_{j} e^{-\frac{1}{h} f}=\frac{\int_{\Omega} \nabla \chi_{k}^{\varepsilon, \varepsilon_{1}} \cdot \widetilde{\phi}_{j} e^{-\frac{1}{h} f}}{\sqrt{\int_{\Omega}\left(\chi_{k}^{\varepsilon, \varepsilon_{1}}\right)^{2} e^{-\frac{2}{h} f}}}=\frac{\int_{B\left(z_{j}, 2 \varepsilon_{1}\right)} \nabla \chi_{k}^{\varepsilon, \varepsilon_{1}} \cdot \widetilde{\phi}_{j} e^{-\frac{1}{h} f}}{\sqrt{\int_{\Omega}\left(\chi_{k}^{\varepsilon, \varepsilon_{1}}\right)^{2} e^{-\frac{2}{h} f}}} .
$$

From (66)-(67), it holds $\arg \min _{\operatorname{supp}} \chi_{k}^{\varepsilon, \varepsilon_{1}} f=\arg \min _{\mathrm{E}_{k}} f$. Thus, using Laplace's method together with the fact that $\min _{\overline{\mathrm{E}_{k}}} f=f\left(x_{k}\right)$, one has in the limit $h \rightarrow 0$ : 


$$
\int_{\Omega}\left(\chi_{k}^{\varepsilon, \varepsilon_{1}}\right)^{2} e^{-\frac{2}{h} f}=(\pi h)^{\frac{d}{2}} e^{-\frac{2}{h} f\left(x_{k}\right)} \sum_{x \in \arg \min _{\mathrm{E}_{k}} f}(\operatorname{det} \operatorname{Hess} f(x))^{-\frac{1}{2}}(1+O(h)) .
$$

Let us now give the estimate of the numerator of the right-hand side of (118).

To prepare this computation, let us first recall that the set $B\left(z_{j}, 2 \varepsilon_{1}\right) \cap\left\{f<f\left(z_{j}\right)\right\}$ has, according to (63), two connected components. Since $z_{j} \in \mathrm{U}_{1}^{\text {ssp }}$ (see Definition 13), exactly one of these two connected components intersects - and is then included in - the critical connected component $\widetilde{\mathbf{j}}\left(x_{k}\right)=\mathrm{E}_{k}$ associated with $x_{k}$ (see Definition 13 and (45)). Moreover, the set $B\left(z_{j}, 2 \varepsilon_{1}\right) \backslash \mathcal{W}_{+}\left(z_{j}\right)$, where the stable manifold $\mathcal{W}_{+}\left(z_{j}\right)$ has been defined in (80), has also two connected components and one of them contains the connected component of $B\left(z_{j}, 2 \varepsilon_{1}\right) \cap\left\{f<f\left(z_{j}\right)\right\}$ which intersects $\mathrm{E}_{k}$, namely $B\left(z_{j}, 2 \varepsilon_{1}\right) \cap \mathrm{E}_{k}$. Let us denote by $\mathrm{B}_{j, k}$ the connected component of $B\left(z_{j}, 2 \varepsilon_{1}\right) \backslash \mathcal{W}_{+}\left(z_{j}\right)$ which contains $\mathrm{E}_{k}$. Since $\operatorname{supp} \widetilde{\phi}_{j} \subset B\left(z_{j}, 2 \varepsilon_{1}\right)$, one has, using (68), $\operatorname{supp}\left(\nabla \chi_{k}^{\varepsilon, \varepsilon_{1}} \cdot \widetilde{\phi}_{j}\right) \subset B\left(z_{j}, 2 \varepsilon_{1}\right) \cap \mathrm{E}_{k} \subset \mathrm{B}_{j, k}$. Therefore, by an integration by parts, it holds:

$$
\begin{aligned}
\int_{\Omega} & \nabla \chi_{k}^{\varepsilon, \varepsilon_{1}} \cdot \widetilde{\phi}_{j} e^{-\frac{1}{h} f}=-\int_{\mathrm{B}_{j, k}} \nabla\left(1-\chi_{k}^{\varepsilon, \varepsilon_{1}}\right) \cdot \widetilde{\phi}_{j} e^{-\frac{1}{h} f} \\
& =-\int_{\mathrm{B}_{j, k}}\left(1-\chi_{k}^{\varepsilon, \varepsilon_{1}}\right) d^{*}\left(e^{-\frac{1}{h} f} \widetilde{\phi}_{j}\right)-\int_{\partial \mathrm{B}_{j, k}}\left(1-\chi_{k}^{\varepsilon, \varepsilon_{1}}\right) \widetilde{\phi}_{j} \cdot n e^{-\frac{1}{h} f} \\
& =-\frac{1}{h} \int_{\mathrm{B}_{j, k}}\left(1-\chi_{k}^{\varepsilon, \varepsilon_{1}}\right) e^{-\frac{1}{h} f} d_{f, h}^{*} \widetilde{\phi}_{j}-\int_{\partial \mathrm{B}_{j, k} \cap \mathcal{W}_{+}\left(z_{j}\right)} \widetilde{\phi}_{j} \cdot n e^{-\frac{1}{h} f},
\end{aligned}
$$

since $\widetilde{\phi}_{j}=0$ on $\partial B\left(z_{j}, 2 \varepsilon_{1}\right)$. From (79), it holds for $h$ small enough,

$$
d_{f, h}^{*} \widetilde{\phi}_{j}=O\left(e^{-\frac{c}{h}}\right) \text { in } L^{2}(\Omega)
$$

where $c>0$ is independent of $h$. Since moreover $f \geq f\left(z_{j}\right)-2 \varepsilon$ on $\mathrm{B}_{j, k} \cap \operatorname{supp}\left(1-\chi_{k}^{\varepsilon, \varepsilon_{1}}\right)$ by (66) and (67), there exist $c^{\prime}>0$ and $\varepsilon_{0}>0$ such that for $\varepsilon \in\left(0, \varepsilon_{0}\right)$, in the limit $h \rightarrow 0$ :

$$
\frac{1}{h} \int_{\mathbf{B}_{j, k}}\left(1-\chi_{k}^{\varepsilon, \varepsilon_{1}}\right) e^{-\frac{1}{h} f} d_{f, h}^{*} \widetilde{\phi}_{j}=O\left(e^{-\frac{1}{h}\left(f\left(z_{j}\right)+c^{\prime}\right)}\right) .
$$

Lastly, using Lemma 40 and the trace theorem, one obtains in the limit $h \rightarrow 0$ :

$$
\begin{aligned}
\int_{\partial \mathrm{B}_{j, k} \cap \mathcal{W}_{+}\left(z_{j}\right)} \widetilde{\phi}_{j} \cdot n e^{-\frac{1}{h} f} & =\int_{\partial \mathrm{B}_{j, k} \cap \mathcal{W}_{+}\left(z_{j}\right)} c_{j}(h) \theta_{j} u_{j, w k b}^{(1)} \cdot n e^{-\frac{1}{h} f}+O\left(h^{\infty} e^{-\frac{1}{h} f\left(z_{j}\right)}\right) \\
& = \pm \frac{c_{j}(h)(\pi h)^{\frac{d-1}{2}}}{\left(\left.\operatorname{det} \operatorname{Hess} f\right|_{\mathcal{W}_{+}\left(z_{j}\right)}\left(z_{j}\right)\right)^{\frac{1}{2}}} e^{-\frac{1}{h} f\left(z_{j}\right)}(1+O(h)) \\
& = \pm \frac{(\pi h)^{\frac{d-2}{4}}\left|\lambda_{-}\left(z_{j}\right)\right|^{\frac{1}{2}}}{\left|\operatorname{det} \operatorname{Hess} f\left(z_{j}\right)\right|^{\frac{1}{4}}} e^{-\frac{1}{h} f\left(z_{j}\right)}(1+O(h))
\end{aligned}
$$

where $\lambda_{-}\left(z_{j}\right)$ denotes the negative eigenvalue of Hess $f\left(z_{j}\right)$. The second equality follows from Laplace's method and from the fact that

$$
u_{j, w k b}^{(1)} \cdot n=\left.e^{-\frac{1}{h} \Phi_{j}}\right|_{\mathcal{W}_{+}\left(z_{j}\right)}\left(\widetilde{a}_{j} \cdot n+O(h)\right)=\left.e^{-\frac{1}{h}\left(f-f\left(z_{j}\right)\right)}\right|_{\mathcal{W}_{+}\left(z_{j}\right)}\left(\widetilde{a}_{j} \cdot n+O(h)\right)
$$


and $\widetilde{a}_{j}\left(z_{j}\right) \cdot n= \pm 1$, see indeed the lines between (82) and (83). The last line follows from (86). The asymptotic estimate of the term $\left\langle\nabla \widetilde{u}_{k}, \widetilde{\psi}_{j}\right\rangle_{L_{w}^{2}}$ is a consequence of the latter estimate together with (118)-(121) which gives in the limit $h \rightarrow 0$ :

$$
\left\langle\nabla \widetilde{u}_{k}, \widetilde{\psi}_{j}\right\rangle_{L_{w}^{2}}= \pm \frac{\pi^{-\frac{1}{2}}\left|\lambda_{-}\left(z_{j}\right)\right|^{\frac{1}{2}} h^{-\frac{1}{2}} e^{-\frac{1}{h}\left(f\left(z_{j}\right)-f\left(x_{k}\right)\right)}}{\left|\operatorname{det} \operatorname{Hess} f\left(z_{j}\right)\right|^{\frac{1}{4}}\left(\sum_{x \in \arg \min _{\mathrm{E}_{k}} f}(\operatorname{det} \operatorname{Hess} f(x))^{-\frac{1}{2}}\right)^{\frac{1}{2}}}(1+O(h)),
$$

where the remainder term $O(h)$ admits a full asymptotic expansion in $h$ (which follows from Laplace's method). This proves Proposition 47 for all $k \in\left\{1, \ldots, \mathrm{m}_{0}^{\Omega}\right\}$ and $j \in\left\{1, \ldots, \mathrm{m}_{1}^{\bar{\Omega}}\right\}$ such that $z_{j} \in \mathbf{j}\left(x_{k}\right) \cap \Omega$.

Step 3. Let us now deal with the computation of the terms $\left\langle\nabla \widetilde{u}_{k}, \widetilde{\psi}_{j}\right\rangle_{L_{w}^{2}}$ for $k \in\left\{1, \ldots, \mathrm{m}_{0}^{\Omega}\right\}$ and $j \in$ $\left\{1, \ldots, \mathrm{m}_{1}^{\bar{\Omega}}\right\}$ when $z_{j} \in \mathbf{j}\left(x_{k}\right) \cap \partial \Omega$. Notice that according to Definition 42 and (65) and by definition of the lexicographic labeling introduced in Definition 41 , this situation can only occur when $k \in\left\{1, \ldots, N_{1}\right\}$. One has

$$
\left\langle\nabla \widetilde{u}_{k}, \widetilde{\psi}_{j}\right\rangle_{L_{w}^{2}}=\int_{\Omega} \nabla \widetilde{u}_{k} \cdot \widetilde{\phi}_{j} e^{-\frac{1}{h} f}=\frac{\int_{\Omega} \nabla \chi_{k}^{\varepsilon, \varepsilon_{1}} \cdot \widetilde{\phi}_{j} e^{-\frac{1}{h} f}}{\sqrt{\int_{\Omega}\left(\chi_{k}^{\varepsilon, \varepsilon_{1}}\right)^{2} e^{-\frac{2}{h} f}}} .
$$

Notice that (119) also holds here for $\int_{\Omega}\left(\chi_{k}^{\varepsilon, \varepsilon_{1}}\right)^{2} e^{-\frac{2}{h} f}$. Since $\operatorname{supp} \widetilde{\phi}_{j} \subset B\left(z_{j}, 2 \varepsilon_{1}\right) \cap \bar{\Omega}$, the numerator of the right-hand side of (122) can be rewritten as

$$
\begin{aligned}
\int_{\Omega} & \nabla \chi_{k}^{\varepsilon, \varepsilon_{1}} \cdot \widetilde{\phi}_{j} e^{-\frac{1}{h} f}=-\int_{B\left(z_{j}, 2 \varepsilon_{1}\right) \cap \bar{\Omega}} \nabla\left(1-\chi_{k}^{\varepsilon, \varepsilon_{1}}\right) \cdot \widetilde{\phi}_{j} e^{-\frac{1}{h} f} \\
= & -\int_{B\left(z_{j}, 2 \varepsilon_{1}\right) \cap \bar{\Omega}}\left(1-\chi_{k}^{\varepsilon, \varepsilon_{1}}\right) d^{*}\left(\widetilde{\phi}_{j} e^{-\frac{1}{h} f}\right)-\int_{\partial\left(B\left(z_{j}, 2 \varepsilon_{1}\right) \cap \bar{\Omega}\right)}\left(1-\chi_{k}^{\varepsilon, \varepsilon_{1}}\right) \widetilde{\phi}_{j} \cdot n e^{-\frac{1}{h} f} \\
= & -\frac{1}{h} \int_{B\left(z_{j}, 2 \varepsilon_{1}\right) \cap \bar{\Omega}}\left(1-\chi_{k}^{\varepsilon, \varepsilon_{1}}\right) e^{-\frac{1}{h} f} d_{f, h}^{*} \widetilde{\phi}_{j}-\int_{\partial B\left(z_{j}, 2 \varepsilon_{1}\right) \cap \partial \Omega} \widetilde{\phi}_{j} \cdot n e^{-\frac{1}{h} f} .
\end{aligned}
$$

From (94), there exists $c>0$ such that for $h$ small enough, $d_{f, h}^{*} \widetilde{\phi}_{j}=O\left(e^{-\frac{c}{h}}\right)$ in $L^{2}(\Omega)$. Since $f \geq f\left(z_{j}\right)-2 \varepsilon$ on $B\left(z_{j}, 2 \varepsilon_{1}\right) \cap \operatorname{supp}\left(1-\chi_{k}^{\varepsilon, \varepsilon_{1}}\right)$ by (64) and (66), there exist $c^{\prime}>0$ and $\varepsilon_{0}>0$ such that for $\varepsilon \in\left(0, \varepsilon_{0}\right)$, in the limit $h \rightarrow 0$ :

$$
\frac{1}{h} \int_{B\left(z_{j}, 2 \varepsilon_{1}\right) \cap \bar{\Omega}}\left(1-\chi_{k}^{\varepsilon, \varepsilon_{1}}\right) e^{-\frac{1}{h} f} d_{f, h}^{*} \widetilde{\phi}_{j}=O\left(e^{-\frac{1}{h}\left(f\left(z_{j}\right)+c^{\prime}\right)}\right) .
$$

Furthermore, applying Proposition 46 with $\Sigma=\partial \Omega$ and $F=1_{\partial B\left(z_{j}, 2 \varepsilon_{1}\right) \cap \partial \Omega}$, one has in the limit $h \rightarrow 0$ :

$$
\int_{\partial B\left(z_{j}, 2 \varepsilon_{1}\right) \cap \partial \Omega} \widetilde{\phi}_{j} \cdot n e^{-\frac{1}{h} f}=\frac{\pi^{\frac{d-1}{4}} \sqrt{2 \partial_{n} f\left(z_{j}\right)}}{\left(\left.\operatorname{det} \operatorname{Hess} f\right|_{\partial \Omega}\left(z_{j}\right)\right)^{1 / 4}} h^{\frac{d-3}{4}} e^{-\frac{1}{h} f\left(z_{1}\right)}(1+O(h)) .
$$

Therefore, injecting the estimates (119), (124), and (125) into (122), one obtains in the limit $h \rightarrow 0$ : 


$$
\left\langle\nabla \widetilde{u}_{k}, \widetilde{\psi}_{j}\right\rangle_{L_{w}^{2}}=-\frac{\pi^{-\frac{1}{4}} \sqrt{2 \partial_{n} f\left(z_{j}\right)} h^{-\frac{3}{4}} e^{-\frac{1}{h}\left(f\left(z_{j}\right)-f\left(x_{k}\right)\right)}}{\left(\left.\operatorname{det} \operatorname{Hess} f\right|_{\partial \Omega}\left(z_{j}\right)\right)^{\frac{1}{4}}\left(\sum_{x \in \arg \min _{\mathrm{E}_{k}} f}(\operatorname{det} \operatorname{Hess} f(x))^{-\frac{1}{2}}\right)^{\frac{1}{2}}}(1+O(h))
$$

where the remainder term $O(h)$ admits a full asymptotic expansion in $h$ (which follows from Laplace's method). This is the desired estimate according to (117) and (106). This ends the proof of Proposition 47.

\subsection{Restricted differential $\nabla: \operatorname{Ran} \pi_{h}^{(0)} \rightarrow \operatorname{Ran} \pi_{h}^{(1)}$}

This section is devoted to the proofs of Theorems 2 and 3. In this section, one also gives lower and upper bounds on the $\mathrm{m}_{0}^{\Omega}$ first eigenvalues of $-L_{f, h}^{D,(0)}$, see Theorem 4 below. As explained above, the idea is to estimate the $\mathrm{m}_{0}^{\Omega}$ singular values of the restricted differential $\nabla: \operatorname{Ran} \pi_{h}^{(0)} \rightarrow \operatorname{Ran} \pi_{h}^{(1)}\left(\operatorname{Ran} \pi_{h}^{(0)}\right.$ and $\operatorname{Ran} \pi_{h}^{(1)}$ being endowed with the $L_{w}^{2}$ inner product) since according to Lemma 23 and Proposition 4, the square of those singular values are the $\mathrm{m}_{0}^{\Omega}$ first eigenvalues of $-\frac{2}{h} L_{f, h}^{D,(0)}$.

This section is organized as follows. Section 4.2.1 is dedicated to the definition of the matrix of the restricted differential $\nabla: \operatorname{Ran} \pi_{h}^{(0)} \rightarrow \operatorname{Ran} \pi_{h}^{(1)}$ and preliminary asymptotic estimates on its coefficients. In Section 4.2.2, lower and upper bounds for the $\mathrm{m}_{0}^{\Omega}$ smallest eigenvalues of $-L_{f, h}^{D,(0)}$ are obtained. Finally, one proves Theorems 2 and 3 in Section 4.2.3.

4.2.1. Matrix of the restricted differential $\nabla: \operatorname{Ran} \pi_{h}^{(0)} \rightarrow \operatorname{Ran} \pi_{h}^{(1)}$

Definition 48. Let us assume that (A0) is satisfied. Let $\left(\widetilde{u}_{k}\right)_{k \in\left\{1, \ldots, \mathbf{m}_{0}^{\Omega}\right\}}$ (resp. $\left.\left(\widetilde{\psi}_{j}\right)_{j \in\left\{1, \ldots, \mathrm{m}_{1}^{\bar{\Omega}}\right\}}\right)$ be the family of quasi-modes for $-L_{f, h}^{D,(0)}$ (resp. $\left.-L_{f, h}^{D,(1)}\right)$ introduced in Definition 42. Let us denote by $S=\left(S_{j, k}\right)_{j, k}$ the $\mathrm{m}_{1}^{\bar{\Omega}} \times \mathrm{m}_{0}^{\Omega}$ matrix defined by: for all $k \in\left\{1, \ldots, \mathrm{m}_{0}^{\Omega}\right\}$ and for all $j \in\left\{1, \ldots, \mathrm{m}_{1}^{\bar{\Omega}}\right\}$

$$
S_{j, k}:=\left\langle\nabla \pi_{h}^{(0)} \widetilde{u}_{k}, \pi_{h}^{(1)} \widetilde{\psi}_{j}\right\rangle_{L_{w}^{2}}
$$

Notice that from (59), it holds for all $k \in\left\{1, \ldots, \mathrm{m}_{0}^{\Omega}\right\}$ and for all $j \in\left\{1, \ldots, \mathrm{m}_{1}^{\bar{\Omega}}\right\}$ :

$$
S_{j, k}=\left\langle\nabla \pi_{h}^{(0)} \widetilde{u}_{k}, \pi_{h}^{(1)} \widetilde{\psi}_{j}\right\rangle_{L_{w}^{2}}=\left\langle\nabla \widetilde{u}_{k}, \pi_{h}^{(1)} \widetilde{\psi}_{j}\right\rangle_{L_{w}^{2}}
$$

Then, using the identity $\left\langle\nabla \widetilde{u}_{k}, \pi_{h}^{(1)} \widetilde{\psi}_{j}\right\rangle_{L_{w}^{2}}=\left\langle\nabla \widetilde{u}_{k}, \widetilde{\psi}_{j}\right\rangle_{L_{w}^{2}}+\left\langle\nabla \widetilde{u}_{k},\left(\pi_{h}^{(1)}-1\right) \widetilde{\psi}_{j}\right\rangle_{L_{w}^{2}}$ together with item 2 in Proposition 43 and Proposition 47, one gets the following estimates on $S$ :

Proposition 49. Let us assume that (A0) is satisfied. Let $S$ be the matrix introduced in Definition 48 . Let $k \in\left\{1, \ldots, \mathrm{m}_{0}^{\Omega}\right\}$ and $j \in\left\{1, \ldots, \mathrm{m}_{1}^{\bar{\Omega}}\right\}$. Then, there exists $\varepsilon_{0}>0$ such that for all $\varepsilon \in\left(0, \varepsilon_{0}\right)$ (where $\varepsilon$ is introduced in (65)), there exists $c>0$ such that in the limit $h \rightarrow 0$ :

$$
\begin{aligned}
\text { if } z_{j} \notin \mathbf{j}\left(x_{k}\right) & , \quad S_{j, k}=O\left(e^{-\frac{1}{h}\left(f\left(\mathbf{j}\left(x_{k}\right)\right)-f\left(x_{k}\right)+c\right)}\right), \\
\text { if } z_{j} \in \mathbf{j}\left(x_{k}\right), \quad S_{j, k} & =\left\langle\nabla \widetilde{u}_{k}, \widetilde{\psi}_{j}\right\rangle_{L_{w}^{2}}\left(1+O\left(e^{-\frac{c}{h}}\right)\right) \\
& =\varepsilon_{j, k} C_{j, k} h^{p_{j, k}} e^{-\frac{1}{h}\left(f\left(\mathbf{j}\left(x_{k}\right)\right)-f\left(x_{k}\right)\right)}(1+O(h))
\end{aligned}
$$

where $\varepsilon_{j, k} \in\{-1,1\}, p_{j, k} \in\left\{-\frac{1}{2},-\frac{3}{4}\right\}$ and $C_{j, k}$ have been introduced in (115), (116), and (117) (see Proposition 47). Moreover, when $z_{j} \in \mathbf{j}\left(x_{k}\right) \cap \partial \Omega$ (and thus $k \in\left\{1, \ldots, \mathrm{N}_{1}\right\}$ ), one has $\varepsilon_{j, k}=-1$.

In order to study the singular values of the matrix $S$, let us introduce the following matrices: 
- Let $\widetilde{S}=\left(\widetilde{S}_{j, k}\right)_{j, k}$ be the real value $\mathrm{m}_{1}^{\bar{\Omega}} \times \mathrm{m}_{0}^{\Omega}$ matrix defined by

$$
\widetilde{S}_{j, k}:= \begin{cases}S_{j, k} & \text { if } z_{j} \in \mathbf{j}\left(x_{k}\right) \\ 0 & \text { if } z_{j} \notin \mathbf{j}\left(x_{k}\right)\end{cases}
$$

- Let $D$ be the diagonal $\mathrm{m}_{0}^{\Omega} \times \mathrm{m}_{0}^{\Omega}$ matrix defined by

$$
\forall k \in\left\{1, \ldots, \mathrm{m}_{0}^{\Omega}\right\}, D_{k, k}:=h^{q_{k}} e^{-\frac{1}{h}\left(f\left(\mathbf{j}\left(x_{k}\right)\right)-f\left(x_{k}\right)\right)} \quad \text { where } q_{k}:=\min _{j, z_{j} \in \mathbf{j}\left(x_{k}\right)}\left\{p_{j, k}\right\},
$$

and where $p_{j, k}$ is defined in (116). Notice that when the assumptions $(\mathbf{A} 1 \mathbf{j})$ and $(\mathbf{A} 2 \mathbf{j})$ are satisfied, one has in the limit $h \rightarrow 0$ (see (51) together with the fact $q_{1}=-\frac{3}{4}$ since $\mathbf{j}\left(x_{1}\right) \cap \partial \Omega=\partial \mathrm{C}_{1} \cap \partial \Omega \neq \emptyset$ which follows from (41) and (A2j)):

$$
D_{1,1}=h^{-\frac{3}{4}} e^{-\frac{1}{h}\left(f\left(\mathbf{j}\left(x_{1}\right)\right)-f\left(x_{1}\right)\right)},
$$

and for some $c>0$ independent of $h$, it holds:

$$
\text { for all } k \in\left\{2, \ldots, \mathrm{m}_{0}^{\Omega}\right\}, \frac{D_{1,1}}{D_{k, k}}=O\left(e^{-\frac{c}{h}}\right) \text {. }
$$

- Let $\widetilde{C}$ be the real value $\mathrm{m}_{1}^{\bar{\Omega}} \times \mathrm{m}_{0}^{\Omega}$ matrix defined by

$$
\widetilde{C}:=\widetilde{S} D^{-1} .
$$

The coefficients of $\widetilde{C}$ are given by $\widetilde{C}_{j, k}=\frac{\widetilde{S}_{j, k}}{D_{k, k}}$, for all $(j, k) \in\left\{1, \ldots, \mathrm{m}_{1}^{\bar{\Omega}}\right\} \times\left\{1, \ldots, \mathrm{m}_{0}^{\Omega}\right\}$. They satisfy, according to Proposition 49 and (128), in the limit $h \rightarrow 0$ :

$$
\widetilde{C}_{j, k}= \begin{cases}\varepsilon_{j, k} C_{j, k} h^{p_{j, k}-q_{k}}(1+O(h)) & \text { if } z_{j} \in \mathbf{j}\left(x_{k}\right), \\ 0 & \text { if } z_{j} \notin \mathbf{j}\left(x_{k}\right),\end{cases}
$$

where $p_{j, k}$ is defined by (116), $\varepsilon_{j, k}$ by (115) and $C_{j, k}$ by (117). From (132), one has when $h \rightarrow 0, \widetilde{C}=O(1)$ which means that there exist $K>0$ and $h_{0}>0$ such that for all $h \in\left(0, h_{0}\right)$ :

$$
\sup _{(j, k) \in\left\{1, \ldots, \mathrm{m}_{1}^{\bar{\Omega}}\right\} \times\left\{1, \ldots, \mathrm{m}_{0}^{\Omega}\right\}}\left|\widetilde{C}_{j, k}\right| \leq K .
$$

Under (A0), by definition of the matrices $S, \widetilde{S}, D$ and $\widetilde{C}$ (see Definition 48 and Equations (127), (128), (131)), there exists $c>0$ such that the matrix $(S-\widetilde{S}) D^{-1}$ satisfies in the limit $h \rightarrow 0$ :

$$
(S-\widetilde{S}) D^{-1}=O\left(e^{-\frac{c}{h}}\right) .
$$

The following lemma will be needed in the sequel.

Lemma 50. Let us assume that (A0) holds. Let $\widetilde{C}$ be the matrix defined in (131). Then, there exist $c>0$ and $h_{0}>0$ such that for all $h \in\left(0, h_{0}\right)$ :

$$
\forall x \in \mathbb{R}^{\mathrm{m}_{0}^{\Omega}}, \quad\|\widetilde{C} x\|_{2} \geq c\|x\|_{2},
$$

where $\|\cdot\|_{2}$ denotes the Euclidean norm on $\mathbb{R}^{K}, K \in \mathbb{N}^{*}$. 
Proof. The proof of Lemma 50 is divided into two steps.

Step 1: Block-diagonal decomposition of $\widetilde{C}$. According to (131), (128) and (127), $\widetilde{C}$ has the form, up to reordering the $z_{i}$ for $i \in\left\{1, \ldots, \mathrm{m}_{1}^{\bar{\Omega}}\right\}$,

$$
\widetilde{C}=\left(\begin{array}{cc}
0 & 0 \\
{[\widetilde{C}]_{a}} & 0 \\
0 & {[\widetilde{C}]_{c}}
\end{array}\right)
$$

where:

- the block matrix $(0,0)$ on the first line corresponds to the rows of $\widetilde{C}$ associated with $j \in\left\{1, \ldots, \mathrm{m}_{1}^{\bar{\Omega}}\right\}$ such that $z_{j} \notin \cup_{k=1}^{\mathrm{m}_{0}^{\Omega}} \mathbf{j}\left(x_{k}\right)$;

- $[\widetilde{C}]_{a}$ is a matrix of size $\operatorname{Card}\left(\cup_{k=1}^{N_{1}} \mathbf{j}\left(x_{k}\right)\right) \times \mathrm{N}_{1}$ (where $\mathrm{N}_{1}$ is defined in (34)). The coefficients $\left([\widetilde{C}]_{a}\right)_{j, k}=$ $\widetilde{C}_{j, k}$ are associated with 0 -forms $\widetilde{u}_{k}$, for $k \in\left\{1, \ldots, \mathrm{N}_{1}\right\}$ (see Definition 42 and (65)) and with 1-forms $\widetilde{\psi}_{j}$ for $j \in\left\{1, \ldots, \mathrm{m}_{1}^{\bar{\Omega}}\right\}$ such that $z_{j} \in \cup_{k=1}^{N_{1}} \mathbf{j}\left(x_{k}\right)$;

- $[\widetilde{C}]_{c}$ is a matrix of size $\operatorname{Card}\left(\cup_{k=\mathrm{N}_{1}+1}^{\mathrm{m}_{0}^{\Omega}} \mathbf{j}\left(x_{k}\right)\right) \times\left(\mathrm{m}_{0}^{\Omega}-\mathrm{N}_{1}\right)$, which has the following block diagonal form:

$$
[\widetilde{C}]_{c}=\left(\begin{array}{cccc}
{[\widetilde{C}]_{c, 1}} & 0 & \ldots & 0 \\
0 & {[\widetilde{C}]_{c, 2}} & \ldots & 0 \\
\vdots & \vdots & \ddots & \vdots \\
0 & 0 & \ldots & {[\widetilde{C}]_{c, N_{1}}}
\end{array}\right)
$$

where for $\ell \in\left\{1, \ldots, \mathrm{N}_{1}\right\},[\widetilde{C}]_{c, \ell}$ is a matrix of size $\operatorname{Card}\left(\bigcup_{k, \mathbf{j}\left(x_{k}\right) \subset \mathrm{c}_{l}} \mathbf{j}\left(x_{k}\right)\right) \times\left(\operatorname{Card}\left(\arg \min \overline{\mathrm{C}_{\ell}} f\right)-\right.$ $1)$, with the convention that $[\widetilde{C}]_{c, \ell}$ does not exist if $\arg \min _{\overline{C_{\ell}}} f=\left\{x_{\ell}\right\}$. Let us recall that for $\ell \in$ $\left\{1, \ldots, N_{1}\right\}, C_{\ell}$ is introduced in Definition 12. For all $\ell \in\left\{1, \ldots, N_{1}\right\},[\widetilde{C}]_{c, \ell}$ contains the non zero terms of $\widetilde{C}$ associated with 0 -forms $\widetilde{u}_{k}$ and 1-forms $\widetilde{\psi}_{j}$ with (i) $\widetilde{u}_{k}$ such that $\operatorname{supp}\left(\widetilde{u}_{k}\right) \subset\left\{\widetilde{\chi}_{\ell}=1\right\}$ (according to (70)); (ii) for those $\widetilde{u}_{k}, j$ is such that $z_{j} \in \mathbf{j}\left(x_{k}\right) \subset \mathrm{C}_{\ell}$. This explains in particular the block structure of $[\widetilde{C}]_{c}$ since by construction $\widetilde{S}_{j, k}=0$ if $z_{j} \notin \mathbf{j}\left(x_{k}\right)$ (see (131) and (127)).

From [28, Section 7.3 and Equation (7.4) in Section 7.2], for all $\ell \in\left\{1, \ldots, \mathrm{N}_{1}\right\}$ there exist $c_{\ell}>0$ and $h_{0}>0$ such that for all $h \in\left(0, h_{0}\right)$ and for all $z \in \mathbb{R}^{\operatorname{Card}\left(\arg \min _{\overline{c_{\ell}}} f\right)-1}$,

$$
\left\|[\widetilde{C}]_{c, \ell} z\right\|_{2} \geq c_{\ell}\|z\|_{2}
$$

Thus, there exist $\alpha>0$ and $h_{0}>0$ such that for all $h \in\left(0, h_{0}\right)$ and for all $z \in \mathbb{R}^{\mathrm{m}_{0}^{\Omega}-\mathrm{N}_{1}}$,

$$
\left\|[\widetilde{C}]_{c} z\right\|_{2} \geq \alpha\|z\|_{2}
$$

For any $x={ }^{t}(y, z) \in \mathbb{R}^{\mathrm{m}_{0}^{\Omega}}\left(y \in \mathbb{R}^{\mathrm{N}_{1}}\right.$ and $\left.z \in \mathbb{R}^{\mathrm{m}_{0}^{\Omega}-\mathrm{N}_{1}}\right)$, it holds

$$
\|\widetilde{C} x\|_{2}^{2}=\left\|[\widetilde{C}]_{a} y\right\|_{2}^{2}+\left\|[\widetilde{C}]_{c} z\right\|_{2}^{2} \geq\left\|[\widetilde{C}]_{a} y\right\|_{2}^{2}+\alpha^{2}\|z\|_{2}^{2} .
$$

Therefore, to prove (135), let us show that there exist $\beta>0$ and $h_{0}>0$ such that for all $h \in\left(0, h_{0}\right)$ and for all $y \in \mathbb{R}^{N_{1}}$,

$$
\left\|[\widetilde{C}]_{a} y\right\|_{2} \geq \beta\|y\|_{2} .
$$


Step 2: Proof of (137). Let us divide the family $\left(C_{k}\right)_{k \in\left\{1, \ldots, N_{1}\right\}}$ into $\mathrm{K}$ groups $\left(\mathrm{K} \leq \mathrm{N}_{1}\right)$ :

$$
\left\{\mathrm{C}_{1}, \ldots, \mathrm{C}_{\mathrm{N}_{1}}\right\}=\bigcup_{\ell=1}^{\mathrm{K}}\left\{\mathrm{C}_{1}^{\ell}, \ldots, \mathrm{C}_{\mathrm{k}_{\ell}}^{\ell}\right\}
$$

which are such that for all $\ell \in\{1, \ldots, \mathrm{K}\}$, the set $\bigcup_{j=1}^{k_{\ell}} \overline{\mathrm{C}_{j}^{\ell}}$ is connected, and for all $q \in\left\{1, \ldots, \mathrm{N}_{1}\right\}$ such that $\mathrm{C}_{q} \notin\left\{\mathrm{C}_{1}^{\ell}, \ldots, \mathrm{C}_{\mathrm{k}_{\ell}}^{\ell}\right\}, \overline{\mathrm{C}_{q}} \cap \bigcup_{j=1}^{k_{\ell}} \overline{\mathrm{C}_{j}^{\ell}}=\emptyset$. Then, by definition of the matrix $[\widetilde{C}]_{a}$ (see Step 1 above), up to a reordering, $[\widetilde{C}]_{a}$ has the block-diagonal form

$$
[\widetilde{C}]_{a}=\left(\begin{array}{cccc}
{[\widetilde{C}]_{a, 1}} & 0 & \ldots & 0 \\
0 & {[\widetilde{C}]_{a, 2}} & \ldots & 0 \\
\vdots & \vdots & \ddots & \vdots \\
0 & 0 & \ldots & {[\widetilde{C}]_{a, \mathrm{~K}}}
\end{array}\right)
$$

where for $\ell \in\{1, \ldots, \mathrm{K}\},[\widetilde{C}]_{a, \ell}$ is a matrix of size $\operatorname{Card}\left(\bigcup_{k=1, x_{k} \in \cup_{j=1}^{k_{\ell}} \mathrm{C}_{j}^{\ell}}^{\mathrm{N}_{1}} \mathbf{j}\left(x_{k}\right)\right) \times k_{\ell}$. For $\ell \in\{1, \ldots, \mathrm{K}\}$, the coefficients $\left([\widetilde{C}]_{a, \ell}\right)_{j, k}=\widetilde{C}_{j, k}$ are associated with 0 -forms $\widetilde{u}_{k}$, for $k \in\left\{1, \ldots, \mathrm{N}_{1}\right\}$ such that $x_{k} \in \cup_{j=1}^{k_{\ell}} \mathrm{C}_{j}^{\ell}$ and with 1-forms $\widetilde{\psi}_{j}$ for $j \in\left\{1, \ldots, \mathrm{m}_{1}^{\bar{\Omega}}\right\}$ such that $z_{j} \in \bigcup_{k=1, x_{k} \in \cup_{j=1}^{k_{\ell}} \mathrm{C}_{j}^{\ell}} \mathbf{j}\left(x_{k}\right)$. Therefore, to prove (137), let us show that for $\ell \in\{1, \ldots, \mathrm{K}\}$, there exist $\beta_{\ell}>0$ and $h_{0}>0$ such that for all $h \in\left(0, h_{0}\right)$ and for all $y \in \mathbb{R}^{k_{\ell}}$,

$$
\left\|[\widetilde{C}]_{a, \ell} y\right\|_{2} \geq \beta_{\ell}\|y\|_{2}
$$

In view of the block structure of $[\widetilde{C}]_{a}$, to prove it, one can assume, without loss of generality, that $\mathrm{K}=1$ which is equivalent to the fact that the set $\bigcup_{j=1}^{N_{1}} \overline{C_{j}}$ is connected. Let us thus assume that $\bigcup_{j=1}^{N_{1}} \overline{C_{j}}$ is connected and let us then write

$$
[\widetilde{C}]_{a}=A+O(h)
$$

where $A$ is a matrix which has the same size as $[\widetilde{C}]_{a}$, and which satisfies, from (132), for all $k \in\left\{1, \ldots, N_{1}\right\}$ and all $j$ such that $z_{j} \in \bigcup_{k=1}^{\mathrm{N}_{1}} \mathbf{j}\left(x_{k}\right)$,

$$
\begin{gathered}
\text { if } \mathbf{j}\left(x_{k}\right) \cap \partial \Omega \neq \emptyset, A_{j, k}= \begin{cases}\varepsilon_{j, k} C_{j, k} & \text { if } z_{j} \in \mathbf{j}\left(x_{k}\right) \cap \partial \Omega, \\
O\left(h^{\frac{1}{4}}\right) & \text { if } z_{j} \in \mathbf{j}\left(x_{k}\right) \cap \Omega, \\
0 & \text { if } z_{j} \notin \mathbf{j}\left(x_{k}\right),\end{cases} \\
\text { if } \mathbf{j}\left(x_{k}\right) \cap \partial \Omega=\emptyset, A_{j, k}= \begin{cases}\varepsilon_{j, k} C_{j, k} & \text { if } z_{j} \in \mathbf{j}\left(x_{k}\right), \\
0 & \text { if } z_{j} \notin \mathbf{j}\left(x_{k}\right),\end{cases}
\end{gathered}
$$

where, we recall, $\varepsilon_{j, k} \in\{-1,1\}$ and $C_{j, k}>0$. To prove (137), it is sufficient to show that (137) holds for $A$ instead of $[\widetilde{C}]_{a}$, i.e. that there exist $\beta>0$ and $h_{0}>0$ such that for all $h \in\left(0, h_{0}\right)$ and for all $y \in \mathbb{R}^{N_{1}}$,

$$
\|A y\|_{2} \geq \beta\|y\|_{2}
$$

Before proving (141), let us label the family $\left(\mathrm{C}_{k}\right)_{k \in\left\{1, \ldots, \mathrm{N}_{1}\right\}}$ as follows. According to Lemma 17, one can assume without loss of generality that $\mathrm{C}_{1}$ is such that there exists $z \in \mathrm{U}_{1}^{\text {ssp }}$ such that

$$
z \in \partial \mathrm{C}_{1} \backslash\left(\cup_{\ell=2}^{N_{1}} \partial \mathrm{C}_{\ell}\right)
$$


Moreover, if $\partial \mathrm{C}_{1} \cap \partial \Omega \neq \emptyset$ (recall that $\partial \mathrm{C}_{1} \cap \partial \Omega \subset \partial \mathrm{C}_{1} \backslash\left(\cup_{\ell=2}^{\mathrm{N}_{1}} \partial \mathrm{C}_{\ell}\right)$ ), one chooses $z$ such that

$$
z \in \partial \mathrm{C}_{1} \cap \partial \Omega
$$

Let us now label $\left(\mathrm{C}_{j}\right)_{j \in\left\{2, \ldots, \mathrm{N}_{1}\right\}}$ such that for all $k \in\left\{1, \ldots, \mathrm{N}_{1}\right\}, \bigcup_{j=1}^{k} \overline{\mathrm{C}_{j}}$ is connected. Let us prove (141) by induction on $k \in\left\{1, \ldots, N_{1}\right\}$ (the proof is similar to the proof made in [28, Section 7.3] in a different context). For $k \in\left\{1, \ldots, \mathrm{N}_{1}\right\}$, one denotes by $\mathcal{P}_{k}$ the following property: there exist $\alpha_{k}>0$ and $h_{0}>0$ such that for all $h \in\left(0, h_{0}\right)$ and for all $y \in \mathbb{R}^{N_{1}}$,

$$
\|A y\|_{2}^{2} \geq \alpha_{k} \sum_{\ell=1}^{k} y_{\ell}^{2}
$$

Let us prove $\mathcal{P}_{1}$. Using (142) and (143) together with (139) and (140), the $j$-th row of $A$ equals

$$
\left(\varepsilon_{j, 1} C_{j, 1}, 0, \ldots, 0\right)
$$

where $j$ is such that $z_{j} \in \partial \mathrm{C}_{1} \backslash\left(\cup_{\ell=2}^{\mathrm{N}_{1}} \partial \mathrm{C}_{\ell}\right)$. Thus, one has for $y \in \mathbb{R}^{\mathrm{N}_{1}},\|A y\|_{2} \geq\left|\varepsilon_{j, 1} C_{j, 1} y_{1}\right|$. Therefore, $\mathcal{P}_{1}$ is satisfied. Let us now assume that $\mathcal{P}_{k}$ is satisfied for some $k \in\left\{1, \ldots, \mathrm{N}_{1}-1\right\}$ and let us prove $\mathcal{P}_{k+1}$.

If $\partial \mathrm{C}_{k+1} \cap \partial \Omega \neq \emptyset$, there exists $j \in\left\{1, \ldots, \mathrm{N}_{1}\right\}$, such that $z_{j} \in \partial \mathrm{C}_{k+1} \cap \partial \Omega$. Thus, using (139), one has $(A y)_{j}=\varepsilon_{j, k+1} C_{j, k+1} y_{k+1}$. Therefore, one obtains $y_{k+1}^{2} C_{j, k+1}^{2}=\left|(A y)_{j}\right|^{2} \leq\|A y\|_{2}^{2}$. Applying the property $\mathcal{P}_{k}$, one gets $\min \left(C_{j, k+1}^{2}, \alpha_{k}\right) \sum_{\ell=1}^{k+1} y_{\ell}^{2} \leq 2\|A y\|_{2}^{2}$. This implies that the property $\mathcal{P}_{k+1}$ is satisfied.

Let us now consider the case $\partial C_{k+1} \cap \partial \Omega=\emptyset$. Using (140) together with the fact that the set $\bigcup_{j=1}^{k+1} \overline{\mathrm{C}}_{j}$ is connected, there exist $\ell \in\{1, \ldots, k\}$ and $j$ such that $z_{j} \in \partial \mathrm{C}_{\ell} \cap \partial \mathrm{C}_{k+1}$. Thus, $(A y)_{j}=$ $A_{j \ell} y_{\ell}+\varepsilon_{j, k+1} C_{j, k+1} y_{k+1}$. As a consequence, since there exists $M>0$ such that $\left|A_{j \ell}\right| \leq M$ for all $h$ small enough, one obtains using the triangular inequality and the property $\mathcal{P}_{k}$,

$$
y_{k+1}^{2} \leq \frac{2}{C_{j, k+1}^{2}}\left(\left|(A y)_{j}\right|^{2}+A_{j \ell}^{2} y_{\ell}^{2}\right) \leq \frac{2\left(1+M^{2} / \alpha_{k}\right)}{C_{j, k+1}^{2}}\|A y\|_{2}^{2} \text {. }
$$

Using again the property $\mathcal{P}_{k}$, one gets that $\min \left(\frac{C_{j, k+1}^{2}}{2\left(1+M^{2} / \alpha_{k}\right)}, \alpha_{k}\right) \sum_{\ell=1}^{k+1} y_{\ell}^{2} \leq 2\|A y\|_{2}^{2}$. Therefore, the property $\mathcal{P}_{k+1}$ is satisfied. This ends the proof of (141) by induction. Together with (136) and (137), one then obtains (135). This concludes the proof of Lemma 50.

As a consequence of (135), the rectangular matrix $\widetilde{C}$ admits a left inverse $\widetilde{C}^{-1}$ which satisfies

$$
\sup _{(j, k) \in\left\{1, \ldots, \mathrm{m}_{0}^{\Omega}\right\} \times\left\{1, \ldots, \mathrm{m}_{1}^{\bar{\Omega}}\right\}}\left|\left(\widetilde{C}^{-1}\right)_{j, k}\right| \leq M
$$

for some $M>0$ independent of $h$. This implies that, using (134) and (131):

$$
S=(I+R) \widetilde{S} \quad \text { where } \quad R=(S-\widetilde{S}) D^{-1} \widetilde{C}^{-1}=O\left(e^{-\frac{c}{h}}\right)
$$

4.2.2. On the $\mathrm{m}_{0}^{\Omega}$ smallest eigenvalues of $-L_{f, h}^{D,(0)}$

This section is dedicated to the proof of the following proposition which gives lower and upper bounds for the $\mathrm{m}_{0}^{\Omega}$ smallest eigenvalues of $-L_{f, h}^{D,(0)}$.

Theorem 4. Let us assume that (A0) holds. Let $\mathbf{j}$ be the map constructed in Section 2.3. Let us reorder the set $\left\{x_{1}, \ldots, x_{\mathrm{m}_{0}^{\Omega}}\right\}$ such that the sequence 


$$
\left(\mathrm{S}_{k}\right)_{k \in\left\{1, \ldots, \mathrm{m}_{0}^{\Omega}\right\}}:=\left(f\left(\mathbf{j}\left(x_{k}\right)\right)-f\left(x_{k}\right)\right)_{k \in\left\{1, \ldots, \mathbf{m}_{0}^{\Omega}\right\}}
$$

is decreasing, and, on any $\mathcal{I} \subset\left\{1, \ldots, \mathrm{m}_{0}^{\Omega}\right\}$ such that $\left(\mathrm{S}_{k}\right)_{k \in \mathcal{I}}$ is constant, the sequence $\left(q_{k}\right)_{k \in \mathcal{I}}$ is decreasing (where the $q_{k}$ 's are introduced in (128)). Finally let us denote by $\lambda_{k, h}$, for $k \in \mathbb{N}^{*}$, the $k$-th eigenvalue of $-L_{f, h}^{D,(0)}$ counted with multiplicity (with these notations, $\lambda_{1, h}=\lambda_{h}$, see (8)). Then, there exist $C>0$ and $h_{0}>0$ such that for all $k \in\left\{1, \ldots, \mathrm{m}_{0}^{\Omega}\right\}$ and for all $h \in\left(0, h_{0}\right)$,

$$
C^{-1} h^{1+2 q_{k}} e^{-\frac{2}{h} S_{k}} \leq \lambda_{k, h} \leq C h^{1+2 q_{k}} e^{-\frac{2}{h} S_{k}} .
$$

The reordering of $\left\{x_{1}, \ldots, x_{\mathrm{m}_{0}^{\Omega}}\right\}$ introduced in (146) is only used in Theorem 4: except in this theorem, the labeling of $\left\{x_{1}, \ldots, x_{\mathrm{m}_{0}^{\Omega}}\right\}$ is the one introduced in Definition 41. A direct consequence of Theorem 4 is the following.

Corollary 51. Let us assume that (A0) and (A1j) are satisfied. Then, the estimate (104) is satisfied.

Before starting the proof of Theorem 4, let us recall the Fan inequalities in Lemma 52 (see for instance [42, Theorem 1.6] or [43]).

Lemma 52. Let $A \in M_{m, m}(\mathbb{C}), B \in M_{m, n}(\mathbb{C})$ and $C \in M_{n, n}(\mathbb{C})$. Then, it holds

$$
\forall i \in\{1, \ldots, n\}, \eta_{i}(A B C) \leq\|A\|\|C\| \eta_{i}(B),
$$

where, for any matrix $T \in M_{m, n}(\mathbb{C}),\|T\|=\eta_{1}(T) \geq \cdots \geq \eta_{n}(T)$ denote the singular values of the matrix $T$ and where $\|T\|:=\sqrt{\max \sigma\left({ }^{t} T T\right)}$ is the spectral norm of $T$.

Let us recall that from item 3 in Proposition 43, there exists $h_{0}>0$ such that for all $h \in\left(0, h_{0}\right)$,

$$
\operatorname{Ran} \pi_{h}^{(0)}=\operatorname{Span}\left(\pi_{h}^{(0)} \widetilde{u}_{k}, k=1, \ldots, \mathrm{m}_{0}^{\Omega}\right) \text { and } \operatorname{Ran} \pi_{h}^{(1)}=\operatorname{Span}\left(\pi_{h}^{(1)} \widetilde{\psi}_{i}, i=1, \ldots, \mathrm{m}_{1}^{\bar{\Omega}}\right)
$$

where the projectors $\pi_{h}^{(0)}$ and $\pi_{h}^{(1)}$ are defined in (60). Let us define $\widetilde{\Upsilon}:=\left(\pi_{h}^{(0)} \widetilde{u}_{k}\right)_{1 \leq k \leq \mathrm{m}_{0}^{\Omega}}$ and $\widetilde{\Psi}:=$ $\left(\pi_{h}^{(1)} \widetilde{\psi}_{j}\right)_{1 \leq j \leq \mathrm{m}_{1}^{\bar{\Omega}}}$. For $i \in\{0,1\}$, let $\mathcal{B}_{i}$ be an orthonormal basis of $\operatorname{Ran} \pi_{h}^{(i)}$ and let us define the matrices

$$
C_{0}:=\operatorname{Mat}_{\widetilde{\Upsilon}} \mathcal{B}_{0} \text { and } C_{1}:=\operatorname{Mat}_{\widetilde{\Psi}} \mathcal{B}_{1}
$$

Notice that from item 3 in Proposition 43, there exist $K>0$ and $h_{0}>0$ such that for all $h \in\left(0, h_{0}\right)$ :

$$
\sup _{(l, k) \in\left\{1, \ldots, \mathrm{m}_{0}^{\Omega}\right\}^{2}}\left|\left(C_{0}\right)_{l, k}\right|+\sup _{(i, j) \in\left\{1, \ldots, \mathrm{m}_{1}^{\bar{\Omega}}\right\}^{2}}\left|\left(C_{1}\right)_{i, j}\right| \leq K
$$

and

$$
\sup _{(l, k) \in\left\{1, \ldots, \mathrm{m}_{0}^{\Omega}\right\}^{2}}\left|\left(C_{0}^{-1}\right)_{l, k}\right|+\sup _{(i, j) \in\left\{1, \ldots, \mathrm{m}_{1}^{\bar{\Omega}}\right\}^{2}}\left|\left(C_{1}^{-1}\right)_{i, j}\right| \leq K .
$$

A consequence of the Fan inequalities is the following.

Lemma 53. Let us assume that (A0) holds. Let us denote by $\lambda_{k, h}$, for $k \in \mathbb{N}^{*}$, the $k$-th eigenvalue of $-L_{f, h}^{D,(0)}$ counted with multiplicity and let $\tilde{S}$ be the matrix defined in (127). Then, there exists $c>0$ such that for all $k \in\left\{1, \ldots, \mathrm{m}_{0}^{\Omega}\right\}$, one has in the limit $h \rightarrow 0$ : 


$$
\lambda_{k, h}=\frac{h}{2}\left[\eta_{\mathbf{m}_{0}^{\Omega}+1-k}\left(\widetilde{S} C_{0}\right)\right]^{2}\left(1+O\left(e^{-\frac{c}{h}}\right)\right)
$$

where $\left\|\widetilde{S} C_{0}\right\|=\eta_{1}\left(\widetilde{S} C_{0}\right) \geq \cdots \geq \eta_{\mathrm{m}_{0}^{\Omega}}\left(\widetilde{S} C_{0}\right)$ denote the singular values of $\widetilde{S} C_{0}$.

Proof. The $\mathrm{m}_{0}^{\Omega} \quad$ smallest eigenvalues of $-L_{f, h}^{D,(0)}$ are the eigenvalues of $-\left.L_{f, h}^{D,(0)}\right|_{\operatorname{Ran} \pi_{h}^{(0)}}=$ $\left.\left.\frac{h}{2} d_{\frac{2 f}{h}, 1}^{*}\right|_{\operatorname{Ran} \pi_{h}^{(1)}} d\right|_{\operatorname{Ran} \pi_{h}^{(0)}}$. Moreover, since the $L_{w}^{2}$-adjoint of $\left.d\right|_{\operatorname{Ran} \pi_{h}^{(0)}}: \operatorname{Ran} \pi_{h}^{(0)} \rightarrow \operatorname{Ran} \pi_{h}^{(1)}$ is $\left.d_{\frac{2 f}{h}, 1}^{*}\right|_{\operatorname{Ran} \pi_{h}^{(1)}}:$ $\operatorname{Ran} \pi_{h}^{(1)} \rightarrow \operatorname{Ran} \pi_{h}^{(0)}$, one has that the $\mathrm{m}_{0}^{\Omega}$ smallest eigenvalues of $-L_{f, h}^{D,(0)}$ are given by $\frac{h}{2}$ times the squares of the singular values of $\left.d\right|_{\operatorname{Ran} \pi_{h}^{(0)}}: \operatorname{Ran} \pi_{h}^{(0)} \rightarrow \operatorname{Ran} \pi_{h}^{(1)}$. Thus, the eigenvalues of $-\left.L_{f, h}^{D,(0)}\right|_{\operatorname{Ran} \pi_{h}^{(0)}}$ are given by $\frac{h}{2}$ times the squares of the singular values of the matrix $Q:=\operatorname{Mat}_{\mathcal{B}_{0}, \mathcal{B}_{1}}\left(\left.d\right|_{\operatorname{Ran} \pi_{h}^{(0)}}\right)$. In addition, by definition of the matrices $C_{0}$ and $C_{1}$ (see (147)), one has $Q={ }^{t} C_{1} S C_{0}$. By (145), it holds $Q={ }^{t} C_{1}(I+R) \widetilde{S} C_{0}$. Furthermore, from (145), there exists $c>0$ such that in the limit $h \rightarrow 0$

$$
\|I+R\|=1+O\left(e^{-\frac{c}{h}}\right), \quad\left\|(I+R)^{-1}\right\|=1+O\left(e^{-\frac{c}{h}}\right)
$$

and from item 3b in Proposition 43,

$$
\left\|{ }^{t} C_{1}\right\|=1+O\left(e^{-\frac{c}{h}}\right), \quad\left\|\left({ }^{t} C_{1}\right)^{-1}\right\|=1+O\left(e^{-\frac{c}{h}}\right),
$$

where we recall that $\|T\|:=\sqrt{\max \sigma\left({ }^{t} T T\right)}$ is the spectral norm of a matrix $T$. Therefore, it follows from the Fan inequalities (see Lemma 52) that the singular values of $Q$ are, up to multiplication by $1+O\left(e^{-\frac{c}{h}}\right)$, the singular values of $\widetilde{S} C_{0}$. This concludes the proof of Lemma 53 .

Remark 54. Notice that in general, the spectral norm of the matrix $C_{0}$ defined in (147) does not equal $1+O\left(e^{-\frac{c}{h}}\right)$ when $h \rightarrow 0$. For instance, in the case when $f$ is a one-dimensional symmetric double-well potential with the saddle point lower than $\min _{\partial \Omega} f$, it can be checked that the Gramian matrix of the functions $\widetilde{u}_{1}$ and $\widetilde{u}_{2}$ introduced in Definition 42 converges when $h \rightarrow 0$ towards the matrix $\left(\begin{array}{cc}1 & c_{1} \\ c_{1} & 1\end{array}\right)$, where $0<c_{1}<1$.

Let us now prove Theorem 4.

Proof of Theorem 4. Theorem 4 is equivalent, according to Lemma 53, to the existence of $C>0$ and $h_{0}>0$ such that for all $k \in\left\{1, \ldots, \mathrm{m}_{0}^{\Omega}\right\}$ and for all $h \in\left(0, h_{0}\right)$

$$
C^{-1} h^{q_{k}} e^{-\frac{1}{h} \mathrm{~S}_{k}} \leq \eta_{\mathrm{m}_{0}^{\Omega}+1-k}\left(\widetilde{S} C_{0}\right) \leq C h^{q_{k}} e^{-\frac{1}{h} \mathrm{~S}_{k}} .
$$

According to (128) and to the ordering of $k \in\left\{1, \ldots, \mathrm{m}_{0}^{\Omega}\right\}$ introduced in the statement of Theorem 4 , the singular values of $D$ satisfy for $h$ small enough (see (128) and (146)):

$$
\forall k \in\left\{1, \ldots, \mathrm{m}_{0}^{\Omega}\right\}, \eta_{\mathrm{m}_{0}^{\Omega}+1-k}(D)=D_{k, k}=h^{q_{k}} e^{-\frac{s_{k}}{h}} .
$$

Using the fact that $\widetilde{S} C_{0}=\widetilde{C} D C_{0}$ (see (131)) together with (133), (148) and Lemma 52, one obtains that for all $k \in\left\{1, \ldots, \mathrm{m}_{0}^{\Omega}\right\}$

$$
\eta_{\mathbf{m}_{0}^{\Omega}+1-k}\left(\widetilde{S} C_{0}\right) \leq\|\widetilde{C}\|\left\|C_{0}\right\| \eta_{\mathbf{m}_{0}^{\Omega}+1-k}(D)=O\left(D_{k, k}\right)
$$

which provides the required upper bound in (151). To obtain a lower bound on the singular values of $\widetilde{S} C_{0}$, we write $D=\widetilde{C}^{-1} \widetilde{S} C_{0} C_{0}^{-1}$. Using (144), (149) and Lemma 52 , one has for all $k \in\left\{1, \ldots, \mathrm{m}_{0}^{\Omega}\right\}$, 


$$
\eta_{\mathrm{m}_{0}^{\Omega}+1-k}(D) \leq\left\|\widetilde{C}^{-1}\right\|\left\|C_{0}^{-1}\right\| \eta_{\mathbf{m}_{0}^{\Omega}+1-k}\left(\widetilde{S} C_{0}\right)=O\left(\eta_{\mathbf{m}_{0}^{\Omega}+1-k}(D)\right)
$$

Then, (151) follows from (152), (154) and (153). This concludes the proof of Theorem 4.

To prove Theorem 2 and to ease upcoming computations, we replace in the Fan inequalities (150) the matrix $C_{0}$ by another matrix which has a simpler form than $C_{0}$ : this is the purpose of Lemma 55 . Before stating Lemma 55, let us choose a specific orthonormal basis $\mathcal{B}_{0}$ of $\operatorname{Ran} \pi_{h}^{(0)}$ to define $C_{0}$ in (147). Recall that when (A1j) holds, the well $\mathrm{C}_{1}$ (see Definition 12) satisfies: for all $x \in \mathrm{U}_{0}^{\Omega} \backslash\left\{x_{1}\right\}$,

$$
f(\mathbf{j}(x))-f(x)<f\left(\mathbf{j}\left(x_{1}\right)\right)-f\left(x_{1}\right) .
$$

Let us define $e_{1}:=\frac{\pi_{h}^{(0)} \widetilde{u}_{1}}{\left\|\pi_{h}^{(0)} \widetilde{u}_{1}\right\|_{L^{2}}}$. According to item 2a in Proposition 43, there exists $c>0$ such that in the limit $h \rightarrow 0, e_{1}=\left(1+O\left(e^{-\frac{c}{h}}\right)\right) \pi_{h}^{(0)} \widetilde{u}_{1}$. Then, let us choose $\left\{e_{2}, \ldots, e_{\mathrm{m}_{0}^{\Omega}}\right\}$ such that $\mathcal{B}_{0}:=\left\{e_{1}, e_{2}, \ldots, e_{\mathrm{m}_{0}^{\Omega}}\right\}$ is an orthonormal basis of $\operatorname{Ran} \pi_{h}^{(0)}$. In that case, the matrix $C_{0}$ defined in (147) satisfies in the limit $h \rightarrow 0$ :

$$
\forall k \in\left\{1, \ldots, \mathrm{m}_{0}^{\Omega}\right\}, \quad\left(C_{0}\right)_{k, 1}= \begin{cases}1+O\left(e^{-\frac{c}{h}}\right) & \text { if } k=1 \\ 0 & \text { if } k>1\end{cases}
$$

Let us now define the $\mathrm{m}_{0}^{\Omega} \times \mathrm{m}_{0}^{\Omega}$ matrix $\widetilde{C}_{0}$ by:

$$
\begin{gathered}
\forall k \in\left\{1, \ldots, \mathrm{m}_{0}^{\Omega}\right\}, \quad\left(\widetilde{C}_{0}\right)_{k, 1}:= \begin{cases}1 & \text { if } k=1, \\
0 & \text { if } k>1,\end{cases} \\
\forall(k, \ell) \in\left\{1, \ldots, \mathrm{m}_{0}^{\Omega}\right\} \times\left\{2, \ldots, \mathrm{m}_{0}^{\Omega}\right\}, \quad\left(\widetilde{C}_{0}\right)_{k, \ell}:=\left(C_{0}\right)_{k, \ell} .
\end{gathered}
$$

Lemma 55. Let us assume that $(\mathbf{A} 0)$ and $(\mathbf{A} 1 \mathbf{j})$ are satisfied. Let us denote by $\lambda_{k, h}$, for $k \in \mathbb{N}^{*}$, the $k$-th eigenvalue of $-L_{f, h}^{D,(0)}$ counted with multiplicity and let $\widetilde{S}$ be the matrix defined in (127). Then, there exists $c>0$ such that in the limit $h \rightarrow 0$ :

$$
\lambda_{k, h}=\frac{h}{2}\left[\eta_{\mathbf{m}_{0}^{\Omega}+1-k}\left(\widetilde{S} \widetilde{C}_{0}\right)\right]^{2}\left(1+O\left(e^{-\frac{c}{h}}\right)\right),
$$

where $\left\|\widetilde{S} \widetilde{C}_{0}\right\|=\eta_{1}\left(\widetilde{S} \widetilde{C}_{0}\right) \geq \cdots \geq \eta_{\mathrm{m}_{0}^{\Omega}}\left(\widetilde{S} \widetilde{C}_{0}\right)$ denote the singular values of $\widetilde{S} \widetilde{C}_{0}$ and $\lambda_{1, h}=\lambda_{h}$ is the principal eigenvalue of $-L_{f, h}^{D,(0)}$ (see (8)).

Proof. Let us prove that there exists $c>0$ such that in the limit $h \rightarrow 0$,

$$
\left\|\left(\widetilde{C}_{0}\right)^{-1} C_{0}\right\|=1+O\left(e^{-\frac{c}{h}}\right) \text { and }\left\|C_{0}^{-1} \widetilde{C}_{0}\right\|=1+O\left(e^{-\frac{c}{h}}\right) .
$$

From (155), the $\mathrm{m}_{0}^{\Omega} \times \mathrm{m}_{0}^{\Omega}$ matrix $C_{0}$ has the form $C_{0}=\left(\begin{array}{cc}1+O\left(e^{-\frac{c}{h}}\right) & {\left[C_{0}\right]_{4}} \\ 0 & {\left[C_{0}\right]_{2}}\end{array}\right)$ for some $c>0$. Moreover, according to (156) and (157), the $\mathrm{m}_{0}^{\Omega} \times \mathrm{m}_{0}^{\Omega}$ matrix $\widetilde{C}_{0}$ has the form $\widetilde{C}_{0}=\left(\begin{array}{ll}1 & {\left[C_{0}\right]_{4}} \\ 0 & {\left[C_{0}\right]_{2}}\end{array}\right)$. Let us recall that by definition of $C_{0}$ (see (147)) and from item $3 \mathrm{a}$ in Proposition $43, C_{0}$ is invertible and thus $\left[C_{0}\right]_{2}$ is invertible. Therefore, one has $\widetilde{C}_{0}^{-1}=\left(\begin{array}{cc}1 & -\left[C_{0}\right]_{4}\left[C_{0}\right]_{2}^{-1} \\ 0 & {\left[C_{0}\right]_{2}^{-1}}\end{array}\right)$ and thus, $\widetilde{C}_{0}^{-1} C_{0}=\left(\begin{array}{cc}1+O\left(e^{-\frac{c}{h}}\right) & 0 \\ 0 & I_{\mathrm{m}_{0}^{\Omega}-1}\end{array}\right)$. This proves (158). Lemma 55 is then a consequence of (158) together with Lemma 53 and Lemma 52. 


\subsubsection{Proofs of Theorems 2 and 3}

This section is dedicated to the proof of Theorem 2 which gives the asymptotic estimate of the principal eigenvalue of $-L_{f, h}^{D,(0)}$ under the assumptions $(\mathbf{A} 1 \mathbf{j})$ and $(\mathbf{A} 2 \mathbf{j})$. The proof of Theorem 3 is similar, and we will only indicate the appropriate modifications (see Remarks 56 and 57).

Proof of Theorem 2. Let us assume that (A0) and (A1j) hold. The spectral gap (104) has already been proved, see Corollary 51. It thus remains to prove (105). The proof of (105) is partly inspired by the analysis led in [28, Section 7.4]. According to Lemma 55, there exists $c>0$ such that in the limit $h \rightarrow 0$ :

$$
\lambda_{1, h}=\frac{h}{2}\left[\eta_{\mathbf{m}_{0}^{\Omega}}\left(\widetilde{S} \widetilde{C}_{0}\right)\right]^{2}\left(1+O\left(e^{-\frac{c}{h}}\right)\right),
$$

where $\widetilde{C}_{0}$ is defined in (156) and (157). Therefore, the analysis of the estimate of $\lambda_{h}$ is then reduced to precisely computing $\eta_{\mathrm{m}_{0}^{\Omega}}\left(\widetilde{S} \widetilde{C}_{0}\right)$. One has:

$$
\eta_{\mathbf{m}_{0}^{\Omega}}\left(\widetilde{S} \widetilde{C}_{0}\right)=\min _{y \in \mathbb{R}^{\mathrm{m}_{0}^{\Omega}},\|y\|_{2}=1}\left\|\widetilde{S} \widetilde{C}_{0} y\right\|_{2}
$$

Let us assume in addition to $(\mathbf{A} \mathbf{0})$ and $(\mathbf{A} 1 \mathbf{j})$ that $(\mathbf{A} 2 \mathbf{j})$ holds. Recall that assumptions $(\mathbf{A} 1 \mathbf{j})$ and $(\mathbf{A} 2 \mathbf{j})$ imply that for all $x \in \mathrm{U}_{0}^{\Omega} \backslash\left\{x_{1}\right\}, f(\mathbf{j}(x))-f(x)<f\left(\mathbf{j}\left(x_{1}\right)\right)-f\left(x_{1}\right)$ and $\partial \mathrm{C}_{1} \cap \partial \Omega \neq \emptyset$. Then, it holds $\mathbf{j}\left(x_{1}\right) \cap \partial \Omega=\partial \mathbf{C}_{1} \cap \partial \Omega \neq \emptyset$ (see (52)). In addition, using Proposition 49 and (127), one has in the limit $h \rightarrow 0$ :

$$
\sum_{j=1}^{\mathrm{m}_{1}^{\bar{\Omega}}} \widetilde{S}_{j, 1}^{2}=\sum_{j: z_{j} \in \mathbf{j}\left(x_{1}\right) \cap \partial \Omega} \widetilde{S}_{j, 1}^{2}+\sum_{j: z_{j} \in \mathbf{j}\left(x_{1}\right) \cap \Omega} \widetilde{S}_{j, 1}^{2},
$$

where:

$$
\left\{\begin{array}{l}
\sum_{j: z_{j} \in \mathbf{j}\left(x_{1}\right) \cap \partial \Omega} \widetilde{S}_{j, 1}^{2}=h^{-\frac{3}{2}}\left(\sum_{j: z_{j} \in \mathbf{j}\left(x_{1}\right) \cap \partial \Omega} C_{j, 1}^{2}\right) e^{-\frac{2}{h}\left(f\left(\mathbf{j}\left(x_{1}\right)\right)-f\left(x_{1}\right)\right)}(1+O(h)), \\
\sum_{j: z_{j} \in \mathbf{j}\left(x_{1}\right) \cap \Omega} \widetilde{S}_{j, 1}^{2}=h^{-1}\left(\sum_{j: z_{j} \in \mathbf{j}\left(x_{1}\right) \cap \Omega} C_{j, 1}^{2}\right) e^{-\frac{2}{h}\left(f\left(\mathbf{j}\left(x_{1}\right)\right)-f\left(x_{1}\right)\right)}(1+O(h)),
\end{array}\right.
$$

where the constants $C_{j, 1}$ are defined in (117) and where all the remainder terms $O(h)$ admit a full asymptotic expansion in $h$.

Let us first obtain an upper bound on $\eta_{\mathrm{m}_{0}}\left(\widetilde{S} \widetilde{C}_{0}\right)$. Let us denote by $y_{0}$ the vector ${ }^{t}(1,0, \ldots, 0)$. Then, it holds from (160), $\eta_{\mathrm{m}_{0}^{\Omega}}\left(\widetilde{S} \widetilde{C}_{0}\right)^{2} \leq\left\|\widetilde{S} C_{0} y_{0}\right\|_{2}^{2}$. Using in addition the fact that from (156), one has $\widetilde{C}_{0} y_{0}=y_{0}$, one obtains

$$
\left[\eta_{\mathrm{m}_{0}^{\Omega}}\left(\widetilde{S} \widetilde{C}_{0}\right)\right]^{2} \leq\left\|\widetilde{S} y_{0}\right\|_{2}^{2}=\sum_{j=1}^{\mathrm{m}_{1}^{\bar{\Omega}}} \widetilde{S}_{j, 1}^{2}
$$

This provides the required upper bound. Notice that (163), (161), and (162) imply that in the limit $h \rightarrow 0$,

$$
\eta_{\mathbf{m}_{0}^{\Omega}}\left(\widetilde{S} \widetilde{C}_{0}\right)=O\left(h^{-\frac{3}{4}} e^{-\frac{1}{h}\left(f\left(\mathbf{j}\left(x_{1}\right)\right)-f\left(x_{1}\right)\right)}\right) .
$$

Let us now give a lower bound on $\eta_{\mathrm{m}_{0}^{\Omega}}\left(\widetilde{S} \widetilde{C}_{0}\right)$. To this end let us consider $y^{*} \in \mathbb{R}^{\mathrm{m}_{0}^{\Omega}}$ with $\left\|y^{*}\right\|_{2}=1$, realizing the minimum in (160). Let us write $y^{*}={ }^{t}\left(y_{\alpha}^{*}, y_{\beta}^{*}\right)$, where $y_{\alpha}^{*} \in \mathbb{R}$ and $y_{\beta}^{*}$ is a row vector of size $\mathrm{m}_{0}^{\Omega}-1$. We claim that there exists $\mu>0$ such that for $h$ small enough,

$$
\left\|y_{\beta}^{*}\right\|_{2}=O\left(e^{-\frac{\mu}{h}}\right) .
$$


Let us prove (165). By definition of $y^{*}$ and according to $(131)$, one has $\eta_{\mathrm{m}_{0}^{\Omega}}\left(\widetilde{S} \widetilde{C}_{0}\right)=\left\|\widetilde{S}_{0} \widetilde{C}^{*}\right\|_{2}=$ $\left\|\widetilde{C} D \widetilde{C}_{0} y^{*}\right\|_{2}$. To prove (165), we use the block structures of the matrices $\widetilde{C}, \widetilde{C}_{0}$ and $D$. Let us recall that from (52) and (53), since (A2j) holds, $\mathbf{k}_{1}^{\partial C_{1}}=\operatorname{Card}\left(\mathbf{j}\left(x_{1}\right) \cap \partial \Omega\right) \geq 1$. Then, according to (131), (128) and (127), the $\mathrm{m}_{1}^{\bar{\Omega}} \times \mathrm{m}_{0}^{\Omega}$ matrix $\widetilde{C}$ has the form, up to reordering the $z_{i}, i \in\left\{1, \ldots, \mathrm{m}_{1}^{\bar{\Omega}}\right\}$,

$$
\widetilde{C}=\left(\begin{array}{cc}
{[\widetilde{C}]_{1}} & 0 \\
{[\widetilde{C}]_{3}} & {[\widetilde{C}]_{2}}
\end{array}\right)
$$

where:

- $[\widetilde{\widetilde{C}}]_{1}$ is a matrix of size $\mathrm{k}_{1}^{\partial \mathrm{C}_{1}} \times 1$ where we recall that $\mathrm{k}_{1}^{\partial \mathrm{C}_{1}}$ is defined in $(53)$. The coefficients $\left([\widetilde{C}]_{1}\right)_{j, 1}=$ $\widetilde{C}_{j, 1}$ are associated with the function $\widetilde{u}_{1}$ (see Definition 42 and (65)) and with 1 -forms $\widetilde{\psi}_{j}$ for $j \in$ $\left\{1, \ldots, \mathrm{k}_{1}^{\partial \mathrm{C}_{1}}\right\}$ (or equivalently, $j$ such that $z_{j} \in \mathbf{j}\left(x_{1}\right) \cap \partial \Omega$ ).

- $[\widetilde{C}]_{3}$ is a matrix of size $\left(\mathrm{m}_{1}^{\bar{\Omega}}-\mathrm{k}_{1}^{\partial \mathrm{C}_{1}}\right) \times 1$. The coefficients $\left([\widetilde{C}]_{3}\right)_{j, k}=\widetilde{C}_{j, k}$ are associated with the function $\widetilde{u}_{1}$ and with 1 -forms $\widetilde{\psi}_{j}$ for $j \in\left\{\mathrm{k}_{1}^{\partial \mathrm{C}_{1}}+1, \ldots, \mathrm{m}_{1}^{\bar{\Omega}}\right\}$ (or equivalently, $j$ such that $z_{j} \notin \mathbf{j}\left(x_{1}\right) \cap \partial \Omega$ ).

- $[\widetilde{C}]_{2}$ is a matrix of size $\left(\mathrm{m}_{1}^{\bar{\Omega}}-\mathrm{k}_{1}^{\partial \mathrm{C}_{1}}\right) \times\left(\mathrm{m}_{0}^{\Omega}-1\right)$. The coefficients $\left([\widetilde{C}]_{2}\right)_{j, k}=\widetilde{C}_{j, k}$ are associated with 0 -forms $\widetilde{u}_{k}$, for $k \in\left\{2, \ldots, \mathrm{m}_{0}^{\Omega}\right\}$ and with 1 -forms $\widetilde{\psi}_{j}$ for $j \in\left\{\mathrm{k}_{1}^{\partial \mathrm{C}_{1}}+1, \ldots, \mathrm{m}_{1}^{\bar{\Omega}}\right\}$ (or equivalently, $j$ such that $\left.z_{j} \notin \mathbf{j}\left(x_{1}\right) \cap \partial \Omega\right)$.

Remark 56. To prove Theorem 3, one uses the block decomposition:

$$
\widetilde{C}=\left(\begin{array}{cc}
{[\widetilde{C}]_{1}} & 0 \\
0 & {[\widetilde{C}]_{2}}
\end{array}\right)
$$

where $[\widetilde{C}]_{1}$ is a matrix of size $\operatorname{Card}\left(\mathbf{j}\left(x_{1}\right)\right) \times 1$ associated with the function $\widetilde{u}_{1}$ and the 1 -forms $\widetilde{\psi}_{j}$ for $j$ such that $z_{j} \in \mathbf{j}\left(x_{1}\right)$, and $[\widetilde{C}]_{2}$ is a matrix of size $\left(\mathrm{m}_{1}^{\bar{\Omega}}-\operatorname{Card}\left(\mathbf{j}\left(x_{1}\right)\right)\right) \times\left(\mathrm{m}_{0}^{\Omega}-1\right)$ associated with the 0 -forms $\widetilde{u}_{k}$, for $k \in\left\{2, \ldots, \mathrm{m}_{0}^{\Omega}\right\}$ and with 1-forms $\widetilde{\psi}_{j}$ for $j$ such that $z_{j} \notin \mathbf{j}\left(x_{1}\right)$. The fact that the lower left and upper right block matrices are zero is a consequence of the following assumption of Theorem 3 : for all $j \in\left\{2, \ldots, \mathrm{N}_{1}\right\}, \partial \mathrm{C}_{1} \cap \partial \mathrm{C}_{j}=\emptyset$.

From (135) and (166), $[\widetilde{C}]_{2}$ is injective and satisfies, for some constant $c>0$ and for all $h>0$ small enough,

$$
\forall x \in \mathbb{R}^{\mathrm{m}_{0}^{\Omega}-1}, \quad\left\|[\widetilde{C}]_{2} x\right\|_{2} \geq c\|x\|_{2} .
$$

This is indeed obvious by applying (135) to the vector ${ }^{t}(0, x)$. Let us now decompose the square matrices $D$ and $\widetilde{C}_{0}$ in blocks which are compatible with the decomposition of $\widetilde{C}$ made in (166). According to (128), (156), and (157), one has

$$
D=\left(\begin{array}{cc}
D_{1,1} & 0 \\
0 & {[D]_{\beta}}
\end{array}\right) \quad \text { and } \widetilde{C}_{0}=\left(\begin{array}{cc}
1 & {\left[\widetilde{C}_{0}\right]_{\gamma}} \\
0 & {\left[\widetilde{C}_{0}\right]_{\beta}}
\end{array}\right),
$$

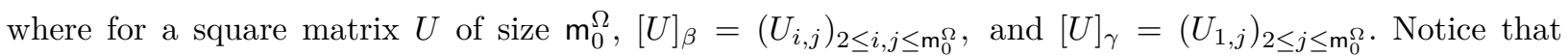
from (129), it holds

$$
D_{1,1}=h^{-\frac{3}{4}} e^{-\frac{1}{h}\left(f\left(\mathbf{j}\left(x_{1}\right)\right)-f\left(x_{1}\right)\right)},
$$

and from (149), there exists $M>0$ such that for $h$ small enough,

$$
\left\|\left[\widetilde{C}_{0}\right]_{\beta}^{-1}\right\| \leq M
$$


We are now in position to prove (165). Let us recall that by definition of $y^{*}$, one has

$$
\eta_{\mathrm{m}_{0}^{\Omega}}\left(\widetilde{S} \widetilde{C}_{0}\right)=\left\|\widetilde{S} \widetilde{C}_{0}^{t}\left(y_{\alpha}^{*}, y_{\beta}^{*}\right)\right\|_{2} \geq\left\|\widetilde{S} \widetilde{C}_{0}{ }^{t}\left(0, y_{\beta}^{*}\right)\right\|_{2}-\left\|\widetilde{S} \widetilde{C}_{0}^{t}\left(y_{\alpha}^{*}, 0\right)\right\|_{2}
$$

Therefore, since $\widetilde{C}_{0}{ }^{t}\left(y_{\alpha}^{*}, 0\right)={ }^{t}\left(y_{\alpha}^{*}, 0\right)$ (see (156)) and $\widetilde{S}=\widetilde{C} D$ (see (131)), one has using (164), (166), and (169) together with the fact that $\left|y_{\alpha}^{*}\right| \leq 1$ and $[\widetilde{C}]_{1}=O(1)$ (see (166) and (133)),

$$
\begin{aligned}
\left\|\widetilde{S} \widetilde{C}_{0}{ }^{t}\left(0, y_{\beta}^{*}\right)\right\|_{2} \leq \eta_{\mathrm{m}_{0}^{\Omega}}\left(\widetilde{S} \widetilde{C}_{0}\right)+\left\|\widetilde{C} D^{t}\left(y_{\alpha}^{*}, 0\right)\right\|_{2} & \leq \eta_{\mathrm{m}_{0}^{\Omega}}\left(\widetilde{S} \widetilde{C}_{0}\right)+\left\|[\widetilde{C}]_{1} D_{1,1} y_{\alpha}^{*}\right\|_{2} \\
& =O\left(h^{-\frac{3}{4}} e^{-\frac{1}{h}\left(f\left(\mathbf{j}\left(x_{1}\right)\right)-f\left(x_{1}\right)\right)}\right) .
\end{aligned}
$$

Moreover, using (169) and since $[\widetilde{C}]_{3}=O(1)$ (see (166) and (133)) and $\left[\widetilde{C}_{0}\right]_{\gamma}=O(1)$ (since $\widetilde{C}_{0}=C_{0}+O\left(e^{-\frac{c}{h}}\right)$ and $C_{0}=O(1)$ see (156), (157), and (148)), one has

$$
\begin{aligned}
\left\|\widetilde{S} \widetilde{C}_{0}{ }^{t}\left(0, y_{\beta}^{*}\right)\right\|_{2} & =\left(\left\|[\widetilde{C}]_{1} D_{1,1}\left[\widetilde{C}_{0}\right]_{\gamma}{ }^{t} y_{\beta}^{*}\right\|_{2}^{2}+\left\|[\widetilde{C}]_{3} D_{1,1}\left[\widetilde{C}_{0}\right]_{\gamma}{ }^{t} y_{\beta}^{*}+[\widetilde{C}]_{2}[D]_{\beta}\left[\widetilde{C}_{0}\right]_{\beta}{ }^{t} y_{\beta}^{*}\right\|_{2}^{2}\right)^{\frac{1}{2}} \\
& \geq\left\|[\widetilde{C}]_{2}[D]_{\beta}\left[\widetilde{C}_{0}\right]_{\beta}{ }^{t} y_{\beta}^{*}\right\|_{2}-\left\|[\widetilde{C}]_{3} D_{1,1}\left[\widetilde{C}_{0}\right]_{\gamma}{ }^{t} y_{\beta}^{*}\right\|_{2} \\
& =\left\|[\widetilde{C}]_{2}[D]_{\beta}\left[\widetilde{C}_{0}\right]_{\beta}{ }^{t} y_{\beta}^{*}\right\|_{2}+O\left(h^{-\frac{3}{4}} e^{-\frac{1}{h}\left(f\left(\mathbf{j}\left(x_{1}\right)\right)-f\left(x_{1}\right)\right)}\right) .
\end{aligned}
$$

Therefore, one deduces from the latter inequality and from (171) and (167) that

$$
\left\|[D]_{\beta}\left[\widetilde{C}_{0}\right]_{\beta} y_{\beta}^{*}\right\|_{2}=O\left(\left\|[\widetilde{C}]_{2}[D]_{\beta}\left[C_{0}\right]_{\beta}{ }^{t} y_{\beta}^{*}\right\|_{2}\right)=O\left(h^{-\frac{3}{4}} e^{-\frac{1}{h}\left(f\left(\mathbf{j}\left(x_{1}\right)\right)-f\left(x_{1}\right)\right)}\right) .
$$

In addition, since $\left[\widetilde{C}_{0}\right]_{\beta}^{-1}=O(1)$ (which follows from $\widetilde{C}_{0}=C_{0}+O\left(e^{-\frac{c}{h}}\right)$, see indeed (156), (157), (149) and $(168))$, and since there exists $c>0$ such that $[D]_{\beta}^{-1}=O\left(e^{\frac{1}{h}\left(f\left(\mathbf{j}\left(x_{1}\right)\right)-f\left(x_{1}\right)-c\right)}\right)$ which follows from (128) and (130), one obtains from (172) that there exists $\mu>0$ such that for $h$ small enough, $\left\|^{t} y_{\beta}^{*}\right\|_{2}=O\left(e^{-\frac{\mu}{h}}\right)$. This ends the proof of (165).

We are now in position to give a lower bound on $\eta_{\mathrm{m}_{0}^{\Omega}}\left(\widetilde{S}_{C_{0}}\right)$. Notice that from (165) together with the fact that $\left\|y^{*}\right\|_{2}=1$, one has

$$
\left|y_{\alpha}^{*}\right|=1+O\left(e^{-\frac{\mu}{h}}\right)
$$

Using (166) and (168), it holds,

$$
\left[\eta_{\mathrm{m}_{0}^{\Omega}}\left(\widetilde{S} \widetilde{C}_{0}\right)\right]^{2} \geq \sum_{j=1}^{\mathrm{k}_{1}^{\partial \mathrm{C}_{1}}}\left(\widetilde{C} D \widetilde{C}_{0} y^{*}\right)_{j}^{2}=\sum_{j=1}^{\mathrm{k}_{1}^{\partial \mathrm{C}_{1}}} D_{1,1}^{2} \widetilde{C}_{j, 1}^{2}\left(y_{\alpha}^{*}+\sum_{\ell=2}^{\mathrm{m}_{0}^{\Omega}}\left(\widetilde{C}_{0}\right)_{1, \ell} y_{\ell}^{*}\right)^{2}
$$

where we recall that $\mathrm{k}_{1}^{\partial \mathrm{C}_{1}}$ is defined by (53). Using in addition (165) and (173) together with the fact that $\widetilde{C}_{0}=O(1)$, there exists $c>0$ such that

$$
\left[\eta_{\mathrm{m}_{0}^{\Omega}}\left(\widetilde{S} \widetilde{C}_{0}\right)\right]^{2} \geq D_{1,1}^{2} \sum_{j=1}^{\mathrm{k}_{1}^{\partial c_{1}}} \widetilde{C}_{j, 1}^{2}\left(1+O\left(e^{-\frac{c}{h}}\right)\right)^{2}
$$

in the limit $h \rightarrow 0$. By definition of $\mathrm{k}_{1}^{\partial \mathrm{C}_{1}}$ (see (53)) it holds 


$$
\begin{aligned}
D_{1,1}^{2} \sum_{j=1}^{\mathrm{k}_{1}^{\partial \mathrm{C}_{1}}} \widetilde{C}_{j, 1}^{2}\left(1+O\left(e^{-\frac{c}{h}}\right)\right)^{2} & =\sum_{j: z_{j} \in \mathbf{j}\left(x_{1}\right) \cap \partial \Omega} \widetilde{C}_{j, 1}^{2} D_{1,1}^{2}\left(1+O\left(e^{-\frac{c}{h}}\right)\right)^{2} \\
& =\sum_{j: z_{j} \in \mathbf{j}\left(x_{1}\right) \cap \partial \Omega} \widetilde{S}_{j, 1}^{2}\left(1+O\left(e^{-\frac{c}{h}}\right)\right)^{2},
\end{aligned}
$$

where the last equality follows from (131). Thus, one obtains the following lower bound:

$$
\left[\eta_{\mathbf{m}_{0}^{\Omega}}\left(\widetilde{S} \widetilde{C}_{0}\right)\right]^{2} \geq \sum_{j: z_{j} \in \mathbf{j}\left(x_{1}\right) \cap \partial \Omega} \widetilde{S}_{j, 1}^{2}\left(1+O\left(e^{-\frac{c}{h}}\right)\right)^{2} .
$$

In conclusion, from (163) and (174), one has for some $c>0$, in the limit $h \rightarrow 0$ :

$$
\sum_{j: z_{j} \in \mathbf{j}\left(x_{1}\right) \cap \partial \Omega} \widetilde{S}_{j, 1}^{2}\left(1+O\left(e^{-\frac{c}{h}}\right)\right)^{2} \leq \eta_{\mathrm{m}_{0}^{\Omega}}^{2}\left(\widetilde{S} \widetilde{C}_{0}\right) \leq \sum_{j=1}^{\mathrm{m}_{1}^{\bar{\Omega}}} \widetilde{S}_{j, 1}^{2} .
$$

Remark 57. In the case of Theorem 3, using the block decomposition of $\widetilde{C}$ given in Remark 56 and a similar reasoning, one gets instead of (175):

$$
\sum_{j: z_{j} \in \mathbf{j}\left(x_{1}\right)} \widetilde{S}_{j, 1}^{2}\left(1+O\left(e^{-\frac{c}{h}}\right)\right)^{2} \leq \eta_{\mathbf{m}_{0}^{\Omega}}^{2}\left(\widetilde{S} \widetilde{C}_{0}\right) \leq \sum_{j=1}^{\mathrm{m}_{1}^{\bar{\Omega}}} \widetilde{S}_{j, 1}^{2} .
$$

This concludes the proof of Theorem 3, using the formulas (161)-(162) to estimate the lower and upper bounds.

Using (161), (162), and the fact that $\mathbf{j}\left(x_{1}\right) \cap \partial \Omega \neq \emptyset$, one gets

$$
\sum_{j=1}^{\mathbf{m}_{1}^{\bar{\Omega}}} \widetilde{S}_{j, 1}^{2}= \begin{cases}\sum_{j: z_{j} \in \mathbf{j}\left(x_{1}\right) \cap \partial \Omega} \widetilde{S}_{j, 1}^{2} & \text { if } \mathbf{j}\left(x_{1}\right) \cap \Omega=\emptyset \\ \sum_{j: z_{j} \in \mathbf{j}\left(x_{1}\right) \cap \partial \Omega} \widetilde{S}_{j, 1}^{2}(1+O(\sqrt{h})) & \text { if } \mathbf{j}\left(x_{1}\right) \cap \Omega \neq \emptyset .\end{cases}
$$

Thus, since $\lambda_{1, h}=\lambda_{h}$, using in addition Proposition 49, (159), and (175), it holds in the limit $h \rightarrow 0$ :

$$
\lambda_{h}=\left\{\begin{array}{l}
\frac{h}{2} \sum_{j: z_{j} \in \mathbf{j}\left(x_{1}\right) \cap \partial \Omega}\left\langle\nabla \widetilde{u}_{1}, \widetilde{\psi}_{j}\right\rangle_{L_{w}^{2}}^{2}\left(1+O\left(e^{-\frac{c}{h}}\right)\right) \text { if } \mathbf{j}\left(x_{1}\right) \cap \Omega=\emptyset, \\
\frac{h}{2} \sum_{j: z_{j} \in \mathbf{j}\left(x_{1}\right) \cap \partial \Omega}\left\langle\nabla \widetilde{u}_{1}, \widetilde{\psi}_{j}\right\rangle_{L_{w}^{2}}^{2}(1+O(\sqrt{h})) \text { if } \mathbf{j}\left(x_{1}\right) \cap \Omega \neq \emptyset .
\end{array}\right.
$$

Then, from (176) and Proposition 47, it holds when $h \rightarrow 0$ :

$$
\lambda_{h}= \begin{cases}\frac{h}{2} \sum_{j: z_{j} \in \mathbf{j}\left(x_{1}\right) \cap \partial \Omega} C_{j, 1}^{2} h^{-\frac{3}{2}} e^{-\frac{2}{h}\left(f\left(\mathbf{j}\left(x_{1}\right)\right)-f\left(x_{1}\right)\right)}(1+O(h)) \quad \text { if } \mathbf{j}\left(x_{1}\right) \cap \Omega=\emptyset, \\ \frac{h}{2} \sum_{j: z_{j} \in \mathbf{j}\left(x_{1}\right) \cap \partial \Omega} C_{j, 1}^{2} h^{-\frac{3}{2}} e^{-\frac{2}{h}\left(f\left(\mathbf{j}\left(x_{1}\right)\right)-f\left(x_{1}\right)\right)}(1+O(\sqrt{h})) \text { if } \mathbf{j}\left(x_{1}\right) \cap \Omega \neq \emptyset,\end{cases}
$$

where the $O(h)$ admits a full asymptotic expansion in $h$. Since $(\mathbf{A} 4 \mathbf{j})$ consists in assuming that $\mathbf{j}\left(x_{1}\right) \cap \Omega=\emptyset$, this concludes the proof of Theorem 2 . 


\section{On the principal eigenfunction of $-L_{f, h}^{D,(0)}$}

This section is dedicated to the proofs of Proposition 58 and Theorem 5 stated below which give respectively the asymptotic behavior in the limit $h \rightarrow 0$ of $\int_{\Omega} u_{h} e^{-\frac{2}{h} f}$ and $\partial_{n} u_{h}$ on $\partial \Omega$.

Proposition 58 gives a sufficient condition to obtain that $u_{h} e^{-\frac{2}{h} f}$ (and thus the quasi-stationary distribution $\nu_{h}$, see Proposition 5) concentrates in only one of the wells $\left(\mathrm{C}_{k}\right)_{k \in\left\{1, \ldots, \mathrm{N}_{1}\right\}}$ when $h \rightarrow 0$ in the $L^{1}(\Omega)$-norm.

Proposition 58. Assume that $(\mathbf{A} 0)$ and $(\mathbf{A} 1 \mathbf{j})$ are satisfied and $\min _{\overline{\mathrm{C}_{1}}} f=\min _{\bar{\Omega}} f$. Let $u_{h}$ be the eigenfunction associated with the principal eigenvalue $\lambda_{h}$ of $-L_{f, h}^{D,(0)}$ (see (8)) which satisfies (9). Let $\mathrm{O}$ be an open subset of $\Omega$. On the one hand, if $\mathrm{O} \cap \arg \min _{\mathrm{C}_{1}} f \neq \emptyset$, one has in the limit $h \rightarrow 0$ :

$$
\int_{\mathrm{O}} u_{h} e^{-\frac{2}{h} f}=h^{\frac{d}{4}} \pi^{\frac{d}{4}} \frac{\sum_{x \in \mathrm{O} \cap \arg \min _{\mathrm{C}_{1}} f}(\operatorname{det} \operatorname{Hess} f(x))^{-\frac{1}{2}}}{\left(\sum_{x \in \arg \min _{\mathrm{C}_{1}} f}(\operatorname{det} \operatorname{Hess} f(x))^{-\frac{1}{2}}\right)^{\frac{1}{2}}} e^{-\frac{1}{h} \min _{\bar{\Omega}} f}(1+O(h)) .
$$

On the other hand, if $\overline{\mathrm{O}} \cap \arg \min _{\mathrm{C}_{1}} f=\emptyset$, then, there exists $c>0$ such that when $h \rightarrow 0$ :

$$
\int_{\mathrm{O}} u_{h} e^{-\frac{2}{h} f}=O\left(e^{-\frac{1}{h}\left(\min _{\bar{\Omega}} f+c\right)}\right)
$$

When (A0) and $(\mathbf{A} 1 \mathbf{j})$ are satisfied and when $\min _{\overline{C_{1}}} f=\min _{\bar{\Omega}} f$ holds, Proposition 58 implies that when $h \rightarrow 0, u_{h} e^{-\frac{2}{h} f}$ concentrates in the $L^{1}$-norm on the global minima of $f$ in $\mathrm{C}_{\max }$. Proposition 58 together with (11) and the fact that $\mathbf{C}_{1}=\mathrm{C}_{\max }$ when (A1j) holds imply Proposition 9 . Notice that when $\mathrm{O}=\Omega$ in Proposition 58, one has from (177), when $h \rightarrow 0$ :

$$
\int_{\Omega} u_{h} e^{-\frac{2}{h} f}=h^{\frac{d}{4}} \pi^{\frac{d}{4}} e^{-\frac{1}{h} \min _{\bar{\Omega}} f}\left(\sum_{x \in \arg \min _{C_{1}} f}(\operatorname{det} \operatorname{Hess} f(x))^{-\frac{1}{2}}\right)^{\frac{1}{2}}(1+O(h)) .
$$

The following theorem shows that, under the hypotheses $(\mathbf{A} 1 \mathbf{j}),(\mathbf{A} \mathbf{j} \mathbf{j})$, and $(\mathbf{A} 3 \mathbf{j})$, the $L_{w}^{1}(\partial \Omega)$-norm of the normal derivative of the principal eigenfunction of $-L_{f, h}^{D,(0)}$ concentrates when $h \rightarrow 0$ on $\partial \Omega \cap \partial \mathrm{C}_{1}$.

Theorem 5. Let us assume that (A0), (A1j), (A2j), and (A3j) are satisfied. Let $u_{h}$ be the eigenfunction associated with the principal eigenvalue $\lambda_{h}$ of $-L_{f, h}^{D,(0)}$ which satisfies $(9)$. Let $F \in L^{\infty}(\partial \Omega, \mathbb{R})$ and $\Sigma$ be an open subset of $\partial \Omega$.

(i) When $\bar{\Sigma} \cap\left\{z_{1}, \ldots, z_{\mathrm{k}_{1} \Omega}\right\}=\emptyset$, one has in the limit $h \rightarrow 0$ :

$$
\int_{\Sigma} F \partial_{n} u_{h} e^{-\frac{2}{h} f}=O\left(e^{-\frac{1}{h}\left(2 \min _{\partial \Omega} f-\min _{\bar{\Omega}} f+c\right)}\right),
$$

where $c>0$ is independent of $h$.

(ii) When $\bar{\Sigma} \cap\left\{z_{1}, \ldots, z_{\mathrm{k}_{1}^{\partial \mathrm{C}_{1}}}\right\}=\emptyset$, one has in the limit $h \rightarrow 0$ :

$$
\int_{\Sigma} F \partial_{n} u_{h} e^{-\frac{2}{h} f}=O\left(h^{\frac{d-6}{4}} e^{-\frac{1}{h}\left(2 \min _{\partial \Omega} f-\min _{\bar{\Omega}} f\right)} \sqrt{\varepsilon_{h}}\right),
$$


where, for some $c>0$ independent of $h$,

$$
\varepsilon_{h}= \begin{cases}\sqrt{h} & \text { or } \\ e^{-\frac{c}{h}} & \text { if }(\mathbf{A} 4 \mathbf{j}) \text { is satisfied. }\end{cases}
$$

(iii) When, for some $i \in\left\{1, \ldots, \mathrm{k}_{1}^{\partial \mathrm{C}_{1}}\right\}, \bar{\Sigma} \cap\left\{z_{1}, \ldots, z_{\mathrm{k}_{1} \mathrm{c}_{1}}\right\}=\left\{z_{i}\right\}, z_{i} \in \Sigma$, and $F$ is $C^{\infty}$ in a neighborhood of $z_{i}$, one has in the limit $h \rightarrow 0$ :

$$
\int_{\Sigma} F \partial_{n} u_{h} e^{-\frac{2}{h} f}=-\left(F\left(z_{i}\right)+O\left(\sqrt{\varepsilon_{h}}\right)+O(h)\right) C_{i, 1} B_{i} h^{\frac{d-6}{4}} e^{-\frac{1}{h}\left(2 \min _{\partial \Omega} f-\min _{\bar{\Omega}} f\right)},
$$

where $\varepsilon_{h}$ satisfies (180) and the constants $B_{i}$ and $C_{i, 1}$ are defined in (117)-(106).

The following simple consequences of Theorem 5 will be useful to prove Theorem 1 . Assume that the assumptions (A0), (A1j), (A2j), and $(\mathbf{A} 3 \mathbf{j})$ are satisfied. Let $F \in L^{\infty}(\partial \Omega, \mathbb{R})$ and $\left(\Sigma_{i}\right)_{i \in\left\{1, \ldots, k_{1}^{\partial \Omega}\right\}}$ be a family of disjoint open subsets of $\partial \Omega$ such that for all $i \in\left\{1, \ldots, \mathrm{k}_{1}^{\partial \Omega}\right\}, z_{i} \in \Sigma_{i}$, where we recall that $\left\{z_{1}, \ldots, z_{\mathrm{k}_{1}^{\partial \Omega}}\right\}=\mathrm{U}_{1}^{\partial \Omega} \cap \arg \min _{\partial \Omega} f($ see $(22))$. Then:

1. There exists $c>0$ such that in the limit $h \rightarrow 0$,

$$
\int_{\partial \Omega} F \partial_{n} u_{h} e^{-\frac{2}{h} f}=\sum_{i=1}^{\mathrm{k}_{1}^{\partial \Omega}} \int_{\Sigma_{i}} F \partial_{n} u_{h} e^{-\frac{2}{h} f}+O\left(e^{-\frac{1}{h}\left(2 \min _{\partial \Omega} f-\min _{\bar{\Omega}} f+c\right)}\right)
$$

and

$$
\sum_{i=\mathrm{k}_{1}^{\partial \mathrm{C}_{1}}}^{\mathrm{k}_{1}^{\partial \Omega}} \int_{+1 \Sigma_{i}} F \partial_{h} u_{h} e^{-\frac{2}{h} f}=O\left(h^{\frac{d-5}{4}} e^{-\frac{1}{h}\left(2 \min _{\partial \Omega} f-\min _{\bar{\Omega}} f\right)}\right)
$$

with the convention $\sum_{i=n}^{m}=0$ if $n>m$ and where we recall that (see (52), (53) and (A3j)), $\left\{z_{1}, \ldots, z_{\mathrm{k}_{1} \mathrm{c}_{1}}\right\}=\partial \mathrm{C}_{1} \cap \partial \Omega \subset \arg \min _{\partial \Omega} f \cap \mathrm{U}_{1}^{\partial \Omega}$. The asymptotic estimate (181) follows from item $(i)$ in Theorem 5 taking $\Sigma=\partial \Omega \backslash \cup_{i=1}^{k_{1}^{\partial \Omega}} \Sigma_{i}$, while (182) follows from item (ii) in Theorem 5 taking $\Sigma=\cup_{i=\mathrm{k}_{1}^{\mathrm{C}} \mathrm{C}_{1}+1}^{\partial \Omega} \Sigma_{i}$

2. Moreover, when, for some $i \in\left\{1, \ldots, \mathrm{k}_{1}^{\partial \mathrm{C}_{1}}\right\}, F$ is $C^{\infty}$ in a neighborhood of $z_{i}$, one has in the limit $h \rightarrow 0$ :

$$
\int_{\Sigma_{i}} F \partial_{n} u_{h} e^{-\frac{2}{h} f}=A_{i}\left(F\left(z_{i}\right)+O\left(h^{\frac{1}{4}}\right)\right) h^{\frac{d-6}{4}} e^{-\frac{1}{h}\left(2 \min _{\partial \Omega} f-\min _{\bar{\Omega}} f\right)},
$$

where

$$
A_{i}=-2 \partial_{n} f\left(z_{i}\right) \pi^{\frac{d-2}{4}}\left(\begin{array}{c}
\left.\operatorname{det} \operatorname{Hess} f\right|_{\partial \Omega}\left(z_{i}\right) \sum_{x \in \arg \min _{\mathcal{C}_{1}} f}(\operatorname{det} \operatorname{Hess} f(x))^{-\frac{1}{2}} \\
)^{-\frac{1}{2}}
\end{array}\right.
$$

This asymptotic equivalent follows from item (iii) in Theorem 5 taking $\Sigma=\Sigma_{i}$ for some $i \in$ $\left\{1, \ldots, k_{1}^{\partial C_{1}}\right\}$. 
3. Lastly, when $(\mathbf{A} 4 \mathbf{j})$ holds (i.e. when $\left.\mathbf{j}\left(x_{1}\right) \subset \partial \Omega\right)$, the remainder term $O\left(h^{\frac{d-5}{4}} e^{-\frac{1}{h}\left(2 f\left(z_{1}\right)-f\left(x_{1}\right)\right)}\right)$ in $(182)$ is of the order $O\left(e^{-\frac{1}{h}\left(2 \min _{\partial \Omega} f-\min _{\bar{\Omega}} f+c\right)}\right)$ for some $c>0$ and the remainder term $O\left(h^{\frac{1}{4}}\right)$ in $(183)$ is of the order $O(h)$ and admits a full asymptotic expansion in $h$.

According to Theorem 5 , when the function $F$ belongs to $C^{\infty}(\partial \Omega, \mathbb{R})$, one has the following equivalent of (181) in the limit $h \rightarrow 0$ :

$$
\int_{\partial \Omega} F \partial_{n} u_{h} e^{-\frac{2}{h} f}=\sum_{i=1}^{\mathrm{k}_{1}^{\partial \mathrm{c}_{1}}} A_{i}\left(F\left(z_{i}\right)+O\left(h^{\frac{1}{4}}\right)\right) h^{\frac{d-6}{4}} e^{-\frac{1}{h}\left(2 \min _{\partial \Omega} f-\min _{\bar{\Omega}} f\right)} .
$$

Remark 59. When the assumption (A4j) is not satisfied, the remainder terms in (182) and (183) are not optimal. In [27, Section C.4.2.2], it is proved on a one-dimensional example, that when the assumption (A4j) is not satisfied, the optimal remainder term in (182) is $O\left(h^{\frac{d-4}{4}} e^{-\frac{1}{h}\left(2 \min _{\partial \Omega} f-\min _{\bar{\Omega}} f\right)}\right)$ and the optimal remainder term in $(183)$ is $O(\sqrt{h})$. In higher-dimension, these optimal remainder terms can be obtained in some specific cases, see [27, Proposition C.40]. It is however unclear how to obtain these optimal remainder terms in general.

This section is organized as follows. In Section 5.1, one proves Proposition 58. Section 5.2 is then dedicated to the proof of Theorem 5 .

\subsection{Proof of Proposition 58}

Let us first give a corollary of Theorem 4 which is used in the proof of Proposition 58 .

Corollary 60. Let us assume that $(\mathbf{A} \mathbf{0})$ and $(\mathbf{A} 1 \mathbf{j})$ are satisfied. Then, there exists $\beta_{0}>0$ such that for all $\beta \in\left(0, \beta_{0}\right)$, there exists $h_{0}>0$ such that for all $h \in\left(0, h_{0}\right)$, the orthogonal projector

$$
\widetilde{\pi}_{h}^{(0)}:=\pi_{\left[0, e^{-\frac{2}{h}\left(f\left(\mathbf{j}\left(x_{1}\right)\right)-f\left(x_{1}\right)-\beta\right)}\right)}\left(-L_{f, h}^{D,(0)}\right)
$$

has rank 1. Moreover, choosing the parameter $\varepsilon>0$ appearing in (65) small enough, there exists $h_{0}>0$ such that for all $h \in\left(0, h_{0}\right)$, one has:

$$
\operatorname{Ran} \widetilde{\pi}_{h}^{(0)}=\operatorname{Span}\left(\widetilde{\pi}_{h}^{(0)} \widetilde{u}_{1}\right),
$$

where the function $\widetilde{u}_{1}$ is introduced in Definition 42.

Proof. The fact that $\operatorname{dim} \operatorname{Ran} \widetilde{\pi}_{h}^{(0)}=1$ is a direct consequence of Corollary 51 . Let us now prove (186). Using Lemma 25, Proposition 4, and using item 2a of Proposition 43, for any $\delta>0$, there exist $\varepsilon>0$ (see (65)), $C>0$ and $h_{0}>0$ such that one has for all $h \in\left(0, h_{0}\right)$,

$$
\begin{aligned}
\left\|\left(1-\widetilde{\pi}_{h}^{(0)}\right) \widetilde{u}_{1}\right\|_{L_{w}^{2}}^{2} & \leq e^{\frac{2}{h}\left(f\left(\mathbf{j}\left(x_{1}\right)\right)-f\left(x_{1}\right)-\beta\right)} \frac{h}{2}\left\|\nabla \widetilde{u}_{1}\right\|_{L_{w}^{2}}^{2} \\
& \leq C e^{\frac{2}{h}\left(f\left(\mathbf{j}\left(x_{1}\right)\right)-f\left(x_{1}\right)-\beta\right)} e^{-\frac{2}{h}\left(f\left(\mathbf{j}\left(x_{1}\right)\right)-f\left(x_{1}\right)-\delta\right)} \leq C e^{-\frac{2}{h}(\beta-\delta)} .
\end{aligned}
$$

Therefore, choosing $\varepsilon>0$ small enough such that $\delta \in(0, \beta)$, there exists $c>0$ and $h_{0}>0$ such that one has for all $h \in\left(0, h_{0}\right)$,

$$
\left\|\widetilde{\pi}_{h}^{(0)} \widetilde{u}_{1}\right\|_{L_{w}^{2}}=1+O\left(e^{-\frac{c}{h}}\right) .
$$


This concludes the proof of (186) and thus the proof of Corollary 60.

Let us now prove Proposition 58.

Proof of Proposition 58. Let us first assume that only $(\mathbf{A} 0)$ and $(\mathbf{A} 1 \mathbf{j})$ are satisfied. As a direct consequence of Corollary 60 and (188), one has (since the functions $u_{h}$ and $\widetilde{u}_{1}$ are non negative),

$$
u_{h}=\frac{\widetilde{\pi}_{h}^{(0)} \widetilde{u}_{1}}{\left\|\widetilde{\pi}_{h}^{(0)} \widetilde{u}_{1}\right\|_{L_{w}^{2}}}=\widetilde{u}_{1}+O\left(e^{-\frac{c}{h}}\right) \text { in } L_{w}^{2}(\Omega) .
$$

Let $O$ be an open subset of $\Omega$. Using (189) and thanks to the Cauchy-Schwarz inequality, one obtains in the limit $h \rightarrow 0$ :

$$
\int_{\mathrm{O}} u_{h} e^{-\frac{2}{h} f}=\int_{\mathrm{O}} \widetilde{u}_{1} e^{-\frac{2}{h} f}+O\left(e^{-\frac{c}{h}}\right) \sqrt{\int_{\mathrm{O}} e^{-\frac{2}{h} f}}=\int_{\mathrm{O}} \widetilde{u}_{1} e^{-\frac{2}{h} f}+O\left(e^{-\frac{1}{h}\left(\min _{\bar{\Omega}} f+c\right)}\right) .
$$

Let us recall that by construction (see Definition 42 and (65)), $\widetilde{u}_{1}=\frac{\chi_{1}^{\varepsilon, \varepsilon_{1}}}{\left\|\chi_{1}^{\varepsilon, \varepsilon_{1}}\right\|_{L_{w}^{2}}}$. Then, from the definition of $\chi_{1}^{\varepsilon, \varepsilon_{1}}$ (see (65) and the lines below) and using Laplace's method, one has in the limit $h \rightarrow 0$,

$$
\int_{\Omega}\left(\chi_{1}^{\varepsilon, \varepsilon_{1}}\right)^{2} e^{-\frac{2}{h} f}=(h \pi)^{\frac{d}{2}} e^{-\frac{2}{h} f\left(x_{1}\right)} \sum_{x \in \arg \min _{\mathcal{C}_{1}} f}(\operatorname{det} \operatorname{Hess} f(x))^{-\frac{1}{2}}(1+O(h)) .
$$

Let us assume that $\mathrm{O} \cap \arg \min _{\mathrm{C}_{1}} f \neq \emptyset$. Then, using Laplace's method, one has when $h \rightarrow 0$,

$$
\int_{\mathrm{O}} \chi_{1}^{\varepsilon, \varepsilon_{1}} e^{-\frac{2}{h} f}=(h \pi)^{\frac{d}{2}} e^{-\frac{2}{h} f\left(x_{1}\right)} \sum_{x \in \mathrm{O} \text { กarg } \min _{\mathrm{C}_{1}} f}(\operatorname{det} \operatorname{Hess} f(x))^{-\frac{1}{2}}(1+O(h)),
$$

where we recall that $x_{1} \in \arg \min _{\mathrm{C}_{1}} f$. Thus, from (190), (191), and (192), one has when $h \rightarrow 0$ :

$$
\begin{aligned}
\int_{\mathrm{O}} u_{h} e^{-\frac{2}{h} f}= & h^{\frac{d}{4}} \pi^{\frac{d}{4}} \frac{\sum_{x \in \mathrm{O} \operatorname{\mathrm {arg}} \min _{\mathrm{C}_{1}} f}(\operatorname{det} \operatorname{Hess} f(x))^{-\frac{1}{2}}}{\left(\sum_{x \in \arg \min _{\mathrm{C}_{1}} f}(\operatorname{det} \operatorname{Hess} f(x))^{-\frac{1}{2}}\right)^{\frac{1}{2}}} e^{-\frac{1}{h} f\left(x_{1}\right)}(1+O(h)) \\
& +O\left(e^{-\frac{1}{h}\left(\min _{\bar{\Omega}} f+c\right)}\right) .
\end{aligned}
$$

Let us assume moreover that $\min _{\overline{C_{1}}} f=\min _{\bar{\Omega}} f$. Then, (177) in Proposition 58 is a consequence of (193).

Let us now consider the case where $\overline{\mathrm{O}} \cap \arg \min _{\mathrm{C}_{1}} f=\emptyset$. Then, it holds

$$
\min _{\overline{\mathrm{O}} \cap \overline{\mathrm{C}_{1}}} f>\min _{\overline{\mathrm{C}_{1}}} f=\min _{\bar{\Omega}} f \text {. }
$$

Since in the limit $h \rightarrow 0, \int_{\mathrm{O}} \chi_{1}^{\varepsilon, \varepsilon_{1}} e^{-\frac{2}{h} f}=O\left(e^{-\frac{2}{h} \min _{\overline{0}} \overline{\bar{C}_{1}} f}\right)$, one obtains using (194), (190), and (191), that there exist $c>0$ and $\tilde{c}>0$ such that when $h \rightarrow 0$ :

$$
\int_{\mathrm{O}} u_{h} e^{-\frac{2}{h} f}=O\left(h^{-\frac{d}{4}} e^{-\frac{2}{h} \min _{\overline{\mathrm{Onc}_{1}} f}} e^{\frac{1}{h} \min _{\bar{\Omega}} f}\right)+O\left(e^{-\frac{1}{h}\left(\min _{\bar{\Omega}} f+c\right)}\right)=O\left(e^{-\frac{1}{h}\left(\min _{\bar{\Omega}} f+\tilde{c}\right)}\right) .
$$

This proves (178) and concludes the proof of Proposition 58. 


\subsection{Proof of Theorem 5}

Let us briefly explain the strategy for the proof of Theorem 5 . The basic idea is to notice that, since $\nabla u_{h}$ belongs to $\operatorname{Ran} \pi_{h}^{(1)}$ (according to (61)), one has for any open set $\Sigma$ of $\partial \Omega$ and for any $L_{w}^{2}$-orthonormal basis $\left(\psi_{1}, \ldots, \psi_{\mathrm{m}_{1}^{\bar{\Omega}}}\right)$ of $\operatorname{Ran} \pi_{h}^{(1)}$,

$$
\int_{\Sigma} F \partial_{n} u_{h} e^{-\frac{2}{h} f}=\sum_{i=1}^{\mathrm{m}_{1}^{\bar{\Omega}}}\left\langle\nabla u_{h}, \psi_{i}\right\rangle_{L_{w}^{2}} \int_{\Sigma} F \psi_{i} \cdot n e^{-\frac{2}{h} f} .
$$

Notice that this decomposition of $\nabla u_{h}$ is valid on $\partial \Omega$. Indeed, for all $i \in\left\{1, \ldots, \mathrm{m}_{1}^{\bar{\Omega}}\right\}, \psi_{i}$ has a smooth trace on $\partial \Omega$ since $\psi_{i} \in \Lambda^{1} C^{\infty}(\bar{\Omega})$ (due to the fact that the eigenforms of $L_{f, h}^{D,(1)}$ belongs to $C^{\infty}(\bar{\Omega})$ and $\pi_{h}^{(1)}$ is a projector onto a finite number of eigenforms of $\left.-L_{f, h}^{D,(1)}\right)$. In the rest of this section, one first introduces such a family $\left\{\psi_{1}, \ldots, \psi_{\mathrm{m}_{1}}\right\}$ using a Gram-Schmidt orthonormalization of the family $\left\{\pi_{h}^{(1)} \widetilde{\psi}_{1}, \ldots, \pi_{h}^{(1)} \widetilde{\psi}_{\mathrm{m}_{1}}\right\}$. Then, one gives estimates of the terms $\left\langle\nabla u_{h}, \psi_{i}\right\rangle_{L_{w}^{2}}$ appearing in (195). Finally, one concludes the proof of Theorem 5 in Section 5.2.3, with estimations of the boundary terms $\int_{\Sigma} F \psi_{i} \cdot n e^{-\frac{2}{h} f}$.

\subsubsection{Gram-Schmidt orthonormalization}

Let us assume that the hypothesis (A0) holds, and assume that $h>0$ is small enough such that the family $\left\{\pi_{h}^{(1)} \widetilde{\psi}_{i}, i=1, \ldots, \mathrm{m}_{1}^{\bar{\Omega}}\right\}$ is independent (which is guaranteed for small $h$ by item $3 \mathrm{~b}$ in Proposition 43 ). Using a Gram-Schmidt procedure, there exists, for all $j \in\left\{1, \ldots, \mathrm{m}_{1}^{\bar{\Omega}}\right\}$, a family $\left(\kappa_{j i}\right)_{i=1, \ldots, j-1} \subset \mathbb{R}^{j-1}$ such that the 1 -forms

$$
f_{j}:=\pi_{h}^{(1)}\left[\widetilde{\psi}_{j}+\sum_{i=1}^{j-1} \kappa_{j i} \widetilde{\psi}_{i}\right]
$$

satisfy: (i) for all $k \in\left\{1, \ldots, \mathrm{m}_{1}^{\bar{\Omega}}\right\}, \operatorname{Span}\left(\left\{f_{i}, i=1, \ldots, k\right\}\right)=\operatorname{Span}\left(\left\{\pi_{h}^{(1)} \widetilde{\psi}_{i}, i=1, \ldots, k\right\}\right)$; (ii) for all $i \neq j,\left\langle f_{i}, f_{j}\right\rangle_{L_{w}^{2}}=0$. One defines moreover, for $j \in\left\{1, \ldots, \mathrm{m}_{1}^{\bar{\Omega}}\right\}$,

$$
Z_{j}:=\left\|f_{j}\right\|_{L_{w}^{2}} \text { and } \psi_{j}:=\frac{1}{Z_{j}} f_{j}
$$

so that $\left(\psi_{j}\right)_{j \in\left\{1, \ldots, \mathrm{m}_{1}^{\bar{\Omega}}\right\}}$ is a $L_{w}^{2}$-orthonormal basis of $\operatorname{Ran} \pi_{h}^{(1)}$. By reasoning by induction (see [1, Section 2] for a similar proof), Proposition 43 easily leads to the following estimates showing in particular that the family $\left(\pi_{h}^{(1)} \widetilde{\psi}_{i}\right)_{i \in\left\{1, \ldots, \mathrm{m}_{1}^{\bar{\Omega}}\right\}}$ is close to the family $\left(\psi_{i}\right)_{i \in\left\{1, \ldots, \mathrm{m}_{1}^{\bar{\Omega}}\right\}}$.

Lemma 61. Let us assume that $(\mathbf{A 0})$ is satisfied. Then, there exists $c>0$ such that for all $j \in$ $\left\{1, \ldots, \mathrm{m}_{1}^{\bar{\Omega}}\right\}, i \in\{1, \ldots, j-1\}$ and $h>0$ small enough, $Z_{j}=1+O\left(e^{-\frac{c}{h}}\right)$ and $\kappa_{j i}=O\left(e^{-\frac{c}{h}}\right)$.

\subsubsection{Estimates of the interaction terms $\left(\left\langle\nabla u_{h}, \psi_{j}\right\rangle_{L_{w}^{2}}\right)_{j \in\left\{1, \ldots, \mathrm{m}_{1}^{\bar{\Omega}}\right\}}$}

Let us start with estimates for the terms $\left\langle\nabla \pi_{h}^{(0)} \widetilde{u}_{k}, \psi_{j}\right\rangle_{L_{w}^{2}}$, where $j \in\left\{1, \ldots, \mathrm{m}_{1}^{\bar{\Omega}}\right\}$ and $k \in\left\{1, \ldots, \mathrm{m}_{0}^{\Omega}\right\}$ (recall that estimates of the terms $\left\langle\nabla \widetilde{u}_{k}, \widetilde{\psi}_{j}\right\rangle_{L_{w}^{2}}$ are given in Proposition 47 ).

Lemma 62. Let us assume that (A0) holds. Then, there exists $c>0$ such that for all $k \in\left\{1, \ldots, \mathrm{m}_{0}^{\Omega}\right\}$, $j \in\left\{1, \ldots, \mathrm{m}_{1}^{\bar{\Omega}}\right\}$, and $h>0$ small enough, it holds: 


$$
\left\langle\nabla \pi_{h}^{(0)} \widetilde{u}_{k}, \psi_{j}\right\rangle_{L_{w}^{2}}= \begin{cases}\left\langle\nabla \widetilde{u}_{k}, \widetilde{\psi}_{j}\right\rangle_{L_{w}^{2}}\left(1+O\left(e^{-\frac{c}{h}}\right)\right) & \text { if } z_{j} \in \mathbf{j}\left(x_{k}\right) \\ O\left(e^{-\frac{1}{h}\left(f\left(\mathbf{j}\left(x_{k}\right)\right)-f\left(x_{k}\right)+c\right)}\right) & \text { if } z_{j} \notin \mathbf{j}\left(x_{k}\right)\end{cases}
$$

Proof. Using (196), (197), and Lemma 61, one has for some $c>0$ and for all $j \in\left\{1, \ldots, \mathrm{m}_{1}^{\bar{\Omega}}\right\}$ and $h>0$ small enough,

$$
\begin{aligned}
& \left\langle\nabla \pi_{h}^{(0)} \widetilde{u}_{k}, \psi_{j}\right\rangle_{L_{w}^{2}}=Z_{j}^{-1}\left[\left\langle\nabla \pi_{h}^{(0)} \widetilde{u}_{k}, \pi_{h}^{(1)} \widetilde{\psi}_{j}\right\rangle_{L_{w}^{2}}+\sum_{i=1}^{j-1} \kappa_{j i}\left\langle\nabla \pi_{h}^{(0)} \widetilde{u}_{k}, \pi_{h}^{(1)} \widetilde{\psi}_{i}\right\rangle_{L_{w}^{2}}\right] \\
& \quad=\left(1+O\left(e^{-\frac{c}{h}}\right)\right)\left[\left\langle\nabla \pi_{h}^{(0)} \widetilde{u}_{k}, \pi_{h}^{(1)} \widetilde{\psi}_{j}\right\rangle_{L_{w}^{2}}+\sum_{i=1}^{j-1} O\left(e^{-\frac{c}{h}}\right)\left\langle\nabla \pi_{h}^{(0)} \widetilde{u}_{k}, \pi_{h}^{(1)} \widetilde{\psi}_{i}\right\rangle_{L_{w}^{2}}\right] .
\end{aligned}
$$

Using Proposition 49, the statement of Lemma 62 follows immediately.

Let us now give asymptotic estimates of the terms $\left\langle\nabla u_{h}, \psi_{j}\right\rangle_{L_{w}^{2}}$ for $j \in\left\{1, \ldots, \mathrm{m}_{1}^{\bar{\Omega}}\right\}$, by proving that $\left\langle\nabla u_{h}, \psi_{j}\right\rangle_{L_{w}^{2}}$ is well approximated by $\left\langle\nabla \pi_{h}^{(0)} \widetilde{u}_{1}, \psi_{j}\right\rangle_{L_{w}^{2}}$, namely that $\pi_{h}^{(0)} \widetilde{u}_{1}$ is an accurate approximation of $u_{h}$ in $H_{w}^{1}(\Omega)$.

Let us recall that when $(\mathbf{A} 0)$ and $(\mathbf{A} 1 \mathbf{j})$ hold, Corollary 60 implies that there exists $\beta_{0}>0$ such that for all $\beta \in\left(0, \beta_{0}\right)$, there exists $h_{0}>0$ such that for all $h \in\left(0, h_{0}\right)$, the orthogonal projector $\tilde{\pi}_{h}^{(0)}=\pi_{\left[0, e^{-\frac{2}{h}\left(f\left(\mathbf{j}\left(x_{1}\right)\right)-f\left(x_{1}\right)-\beta\right)}\right)}\left(-L_{f, h}^{D,(0)}\right)$ has rank 1. Therefore, $\tilde{\pi}_{h}^{(0)}$ is the orthogonal projector onto Span $\left(u_{h}\right)$. Moreover, from the second equality in (189) and item 3a in Proposition 43, one has $u_{h}=\pi_{h}^{(0)} \widetilde{u}_{1}+O\left(e^{-\frac{c}{h}}\right)$ in $L_{w}^{2}(\Omega)$. Therefore, $\pi_{h}^{(0)} \widetilde{u}_{1}$ is an accurate approximation of $u_{h}$ in $L_{w}^{2}(\Omega)$. The following lemma extends this result in $H_{w}^{1}(\Omega)$ when assuming $(\mathbf{A} \mathbf{j} \mathbf{j})$ in addition to $(\mathbf{A} \mathbf{0})$ and $(\mathbf{A} 1 \mathbf{j})$.

Lemma 63. Assume that (A0), (A1j), and (A2j) hold. Then, it holds in the limit $h \rightarrow 0$ :

$$
\left\|\nabla \pi_{h}^{(0)} \widetilde{u}_{1}\right\|_{L_{w}^{2}}^{2}=\frac{2}{h} \lambda_{h}\left(1+O\left(\varepsilon_{h}\right)\right)
$$

and

$$
\left\|\nabla\left(\pi_{h}^{(0)}-\widetilde{\pi}_{h}^{(0)}\right) \widetilde{u}_{1}\right\|_{L_{w}^{2}}^{2}=\frac{2}{h} \lambda_{h} O\left(\varepsilon_{h}\right)=O\left(h^{-\frac{3}{2}} e^{-\frac{2}{h}\left(f\left(\mathbf{j}\left(x_{1}\right)\right)-f\left(x_{1}\right)\right)} \varepsilon_{h}\right),
$$

where $\varepsilon_{h}$ satisfies (180).

Proof. Applying the Parseval's identity to $\nabla \pi_{h}^{(0)} \widetilde{u}_{1} \in \operatorname{Ran} \pi_{h}^{(1)}$ (see (59)), one gets $\left\|\nabla \pi_{h}^{(0)} \widetilde{u}_{1}\right\|_{L_{w}^{2}}^{2}=$ $\sum_{j=1}^{\mathrm{m}_{1}^{\bar{\Omega}}}\left\langle\nabla \pi_{h}^{(0)} \widetilde{u}_{1}, \psi_{j}\right\rangle_{L_{w}^{2}}^{2}$. Using Lemma 62 , there exists consequently $c>0$ such that for all $h>0$ small enough,

$$
\left\|\nabla \pi_{h}^{(0)} \widetilde{u}_{1}\right\|_{L_{w}^{2}}^{2}=\sum_{j: z_{j} \in \mathbf{j}\left(x_{1}\right)}\left\langle\nabla \widetilde{u}_{1}, \widetilde{\psi}_{j}\right\rangle_{L_{w}^{2}}^{2}\left(1+O\left(e^{-\frac{c}{h}}\right)\right)
$$

Using in addition (176), one then obtains the first part of Lemma 63, i.e.:

$$
\left\|\nabla \pi_{h}^{(0)} \widetilde{u}_{1}\right\|_{L_{w}^{2}}^{2}=\frac{2}{h} \lambda_{h}\left(1+O\left(\varepsilon_{h}\right)\right)
$$

where, in the limit $h \rightarrow 0, \varepsilon_{h}$ satisfies (180). 
Let us recall that from (189) and (188), one has for any $h$ small enough

$$
u_{h}=\frac{\widetilde{\pi}_{h}^{(0)} \widetilde{u}_{1}}{\left\|\widetilde{\pi}_{h}^{(0)} \widetilde{u}_{1}\right\|_{L_{w}^{2}}} \text { where }\left\|\widetilde{\pi}_{h}^{(0)} \widetilde{u}_{1}\right\|_{L_{w}^{2}}=1+O\left(e^{-\frac{c}{h}}\right) .
$$

Now, since the projectors $\pi_{h}^{(0)}$ and $\widetilde{\pi}_{h}^{(0)}$ commute with $L_{f, h}^{D,(0)}$, and $\widetilde{\pi}_{h}^{(0)} \pi_{h}^{(0)}=\widetilde{\pi}_{h}^{(0)}$, one has

$$
\begin{aligned}
\frac{h}{2}\left\|\nabla\left(\pi_{h}^{(0)}-\widetilde{\pi}_{h}^{(0)}\right) \widetilde{u}_{1}\right\|_{L_{w}^{2}}^{2} & =\left\langle\left(\pi_{h}^{(0)}-\widetilde{\pi}_{h}^{(0)}\right) \widetilde{u}_{1},-L_{f, h}^{D,(0)}\left(\pi_{h}^{(0)}-\widetilde{\pi}_{h}^{(0)}\right) \widetilde{u}_{1}\right\rangle_{L_{w}^{2}} \\
& =\left\langle\pi_{h}^{(0)} \widetilde{u}_{1},-L_{f, h}^{D,(0)}\left(\pi_{h}^{(0)}-\widetilde{\pi}_{h}^{(0)}\right) \widetilde{u}_{1}\right\rangle_{L_{w}^{2}} \\
& =\left\langle\pi_{h}^{(0)} \widetilde{u}_{1},-L_{f, h}^{D,(0)} \pi_{h}^{(0)} \widetilde{u}_{1}\right\rangle_{L_{w}^{2}}-\left\langle\widetilde{\pi}_{h}^{(0)} \widetilde{u}_{1},-L_{f, h}^{D,(0)} \widetilde{\pi}_{h}^{(0)} \widetilde{u}_{1}\right\rangle_{L_{w}^{2}} \\
& =\frac{h}{2}\left\|\nabla \pi_{h}^{(0)} \widetilde{u}_{1}\right\|_{L_{w}^{2}}^{2}-\lambda_{h}\left(1+O\left(e^{-\frac{c}{h}}\right)\right),
\end{aligned}
$$

where the last line follows from (199). Using in addition (198), one obtains in the limit $h \rightarrow 0$ :

$$
\frac{h}{2}\left\|\nabla\left(\pi_{h}^{(0)}-\widetilde{\pi}_{h}^{(0)}\right) \widetilde{u}_{1}\right\|_{L_{w}^{2}}^{2}=\lambda_{h}\left(1+O\left(\varepsilon_{h}\right)\right)-\lambda_{h}\left(1+O\left(e^{-\frac{c}{h}}\right)\right)=\lambda_{h} O\left(\varepsilon_{h}\right),
$$

which proves Lemma 63, using also the asymptotic estimate of $\lambda_{h}$ given in Theorem 2, see (105).

We are now in position to estimate the interaction terms $\left(\left\langle\nabla u_{h}, \psi_{j}\right\rangle_{L_{w}^{2}}\right)_{j \in\left\{1, \ldots, \mathrm{m}_{1}^{\bar{\Omega}}\right\}}$.

Corollary 64. Let us assume that $(\mathbf{A} 0),(\mathbf{A} 1 \mathbf{j})$ and $(\mathbf{A} 2 \mathbf{j})$ hold. Let $u_{h}$ be the eigenfunction associated with the principal eigenvalue $\lambda_{h}$ of $-L_{f, h}^{D,(0)}$ (see (8)) which satisfies (9). Then, in the limit $h \rightarrow 0$ :

(i) for all $j \in\left\{1, \ldots, \mathrm{m}_{1}^{\bar{\Omega}}\right\}$ such that $z_{j} \in \mathbf{j}\left(x_{1}\right) \cap \partial \Omega$ (i.e. for all $j \in\left\{1, \ldots, \mathrm{k}_{1}^{\partial \mathrm{C}_{1}}\right\}$, see (52) and (53)),

$$
\begin{aligned}
\left\langle\nabla u_{h}, \psi_{j}\right\rangle_{L_{w}^{2}} & =\left\langle\nabla \widetilde{u}_{1}, \widetilde{\psi}_{j}\right\rangle_{L_{w}^{2}}\left(1+O\left(\sqrt{\varepsilon_{h}}\right)\right) \\
& =-C_{j, 1} h^{-\frac{3}{4}} e^{-\frac{1}{h}\left(f\left(\mathbf{j}\left(x_{1}\right)\right)-f\left(x_{1}\right)\right)}\left(1+O\left(\sqrt{\varepsilon_{h}}\right)+O(h)\right),
\end{aligned}
$$

(ii) for all $j \in\left\{1, \ldots, \mathrm{m}_{1}^{\bar{\Omega}}\right\}$ such that $z_{j} \in \mathbf{j}\left(x_{1}\right) \cap \Omega$,

$$
\begin{aligned}
\left\langle\nabla u_{h}, \psi_{j}\right\rangle_{L_{w}^{2}} & =\left\langle\nabla \widetilde{u}_{1}, \widetilde{\psi}_{j}\right\rangle_{L_{w}^{2}}\left(1+O\left(h^{-\frac{1}{4}} \sqrt{\varepsilon_{h}}\right)\right) \\
& =O\left(h^{-\frac{1}{2}} e^{-\frac{1}{h}\left(f\left(\mathbf{j}\left(x_{1}\right)\right)-f\left(x_{1}\right)\right)}\right),
\end{aligned}
$$

(iii) and for all $j \in\left\{1, \ldots, \mathrm{m}_{1}^{\bar{\Omega}}\right\}$ such that $z_{j} \notin \mathbf{j}\left(x_{1}\right)$,

$$
\left\langle\nabla u_{h}, \psi_{j}\right\rangle_{L_{w}^{2}}=O\left(h^{-\frac{3}{4}} e^{-\frac{1}{h}\left(f\left(\mathbf{j}\left(x_{1}\right)\right)-f\left(x_{1}\right)\right)} \sqrt{\varepsilon_{h}}\right),
$$

where $\varepsilon_{h}$ satisfies (180).

Proof. Using (199), there exists $c>0$ such that for all $j \in\left\{1, \ldots, \mathrm{m}_{1}^{\bar{\Omega}}\right\}$, in the limit $h \rightarrow 0$ :

$$
\left\langle\nabla u_{h}, \psi_{j}\right\rangle_{L_{w}^{2}}=\left\langle\nabla \widetilde{\pi}_{h}^{(0)} \widetilde{u}_{1}, \psi_{j}\right\rangle_{L_{w}^{2}}\left(1+O\left(e^{-\frac{c}{h}}\right)\right) .
$$


In addition, using the Cauchy-Schwarz inequality and the second statement in Lemma 63, it holds for all $j \in\left\{1, \ldots, \mathrm{m}_{1}^{\bar{\Omega}}\right\}$, in the limit $h \rightarrow 0$ :

$$
\begin{aligned}
\left\langle\nabla \widetilde{\pi}_{h}^{(0)} \widetilde{u}_{1}, \psi_{j}\right\rangle_{L_{w}^{2}} & =\left\langle\nabla \pi_{h}^{(0)} \widetilde{u}_{1}, \psi_{j}\right\rangle_{L_{w}^{2}}+\left\langle\nabla\left(\widetilde{\pi}_{h}^{(0)}-\pi_{h}^{(0)}\right) \widetilde{u}_{1}, \psi_{j}\right\rangle_{L_{w}^{2}} \\
& =\left\langle\nabla \pi_{h}^{(0)} \widetilde{u}_{1}, \psi_{j}\right\rangle_{L_{w}^{2}}+O\left(h^{-\frac{3}{4}} e^{-\frac{1}{h}\left(f\left(\mathbf{j}\left(x_{1}\right)\right)-f\left(x_{1}\right)\right)} \sqrt{\varepsilon_{h}}\right),
\end{aligned}
$$

where $\varepsilon_{h}$ is of the order given by (180). Then, the statement of Corollary 64 follows by injecting (201) into (200) and by using the estimates of the terms $\left\langle\nabla \pi_{h}^{(0)} \widetilde{u}_{1}, \psi_{j}\right\rangle_{L_{w}^{2}}\left(j \in\left\{1, \ldots, \mathrm{m}_{1}^{\bar{\Omega}}\right\}\right)$ given in Lemma 62.

5.2.3. Estimates of the boundary terms $\left(\int_{\Sigma} F \psi_{j} \cdot n e^{-\frac{2}{h} f}\right)_{j \in\left\{1, \ldots, \mathrm{m}_{1}^{\bar{\Omega}}\right\}}$

Proposition 65. Let us assume that $(\mathbf{A} 0)$ is satisfied. Let us consider $i \in\left\{1, \ldots, \mathrm{m}_{1}^{\bar{\Omega}}\right\}$, an open set $\Sigma$ of $\partial \Omega$, and $F \in L^{\infty}(\partial \Omega, \mathbb{R})$. Then, there exists $c>0$ such that in the limit $h \rightarrow 0$ :

$$
\int_{\Sigma} F \psi_{i} \cdot n e^{-\frac{2}{h} f}= \begin{cases}O\left(e^{-\frac{1}{h}\left(\min _{\partial \Omega} f+c\right)}\right) & \text { if } i \in\left\{\mathrm{k}_{1}^{\partial \Omega}+1, \ldots, \mathrm{m}_{1}^{\bar{\Omega}}\right\} \\ O\left(e^{-\frac{1}{h}\left(\min _{\partial \Omega} f+c\right)}\right) & \text { if } i \in\left\{1, \ldots, \mathrm{k}_{1}^{\partial \Omega}\right\} \text { and } z_{i} \notin \bar{\Sigma} \\ O\left(h^{\frac{d-3}{4}} e^{-\frac{1}{h} \min _{\partial \Omega} f}\right) & \text { if } i \in\left\{1, \ldots, \mathrm{k}_{1}^{\partial \Omega}\right\} \text { and } z_{i} \in \bar{\Sigma}\end{cases}
$$

where we recall that $\left\{z_{1}, \ldots, z_{\mathrm{k}_{1}^{\partial \Omega}}\right\}=\mathrm{U}_{1}^{\partial \Omega} \cap \arg \min _{\partial \Omega} f\left(\right.$ see (22)). Moreover, when $i \in\left\{1, \ldots, \mathrm{k}_{1}^{\partial \Omega}\right\}, z_{i} \in \Sigma$, and $F$ is $C^{\infty}$ in a neighborhood of $z_{i}$, it holds in the limit $h \rightarrow 0$ :

$$
\int_{\Sigma} F \psi_{i} \cdot n e^{-\frac{2}{h} f}=h^{\frac{d-3}{4}} e^{-\frac{1}{h} \min _{\partial \Omega} f}\left(B_{i} F\left(z_{i}\right)+O(h)\right),
$$

where the constant $B_{i}$ is defined in (106).

Proof. Let $F \in L^{\infty}(\partial \Omega, \mathbb{R})$. Using (196), (197), the trace theorem, and the Cauchy-Schwarz inequality, one has for all $j \in\left\{1, \ldots, \mathrm{m}_{1}^{\bar{\Omega}}\right\}$,

$$
\begin{aligned}
Z_{j} \int_{\Sigma} F \psi_{j} \cdot n e^{-\frac{2}{h} f}= & \int_{\Sigma} F \widetilde{\psi}_{j} \cdot n e^{-\frac{2}{h} f}+\int_{\Sigma} F\left(\left(\pi_{h}^{(1)}-1\right) \widetilde{\psi}_{j}\right) \cdot n e^{-\frac{2}{h} f} \\
& +\sum_{i=1}^{j-1} \kappa_{j i}\left[\int_{\Sigma} F \widetilde{\psi}_{i} \cdot n e^{-\frac{2}{h} f}+\int_{\Sigma} F\left(\left(\pi_{h}^{(1)}-1\right) \widetilde{\psi}_{i}\right) \cdot n e^{-\frac{2}{h} f}\right] \\
= & \int_{\Sigma} F \widetilde{\psi}_{j} \cdot n e^{-\frac{2}{h} f}+\left\|\left(1-\pi_{h}^{(1)}\right) \widetilde{\psi}_{j}\right\|_{H_{w}^{1}} O\left(h^{-1} e^{-\frac{\min _{\partial \Omega} f}{h}}\right) \\
& +\sum_{i=1}^{j-1} \kappa_{j i}\left[\int_{\Sigma} F \widetilde{\psi}_{i} \cdot n e^{-\frac{2}{h} f}+\left\|\left(1-\pi_{h}^{(1)}\right) \widetilde{\psi}_{i}\right\|_{H_{w}^{1}} O\left(h^{-1} e^{\left.-\frac{\min _{\partial \Omega} f}{h}\right)}\right)\right] .
\end{aligned}
$$

From Lemma 61 and item $2 \mathrm{~b}$ in Proposition 43, there exists $c>0$ such that for all $j \in\left\{1, \ldots, \mathrm{m}_{1}^{\bar{\Omega}}\right\}, i \in$ $\{1, \ldots, j-1\}$, in the limit $h \rightarrow 0, Z_{j}=1+O\left(e^{-\frac{c}{h}}\right), \kappa_{j i}=O\left(e^{-\frac{c}{h}}\right)$, and $\left\|\left(1-\pi_{h}^{(1)}\right) \widetilde{\psi}_{j}\right\|_{H_{w}^{1}}=O\left(e^{-\frac{c}{h}}\right)$.

Therefore, using Proposition 46, there exists $c>0$ such that for all $j \in\left\{1, \ldots, \mathrm{m}_{1}^{\bar{\Omega}}\right\}$, in the limit $h \rightarrow 0$ :

$$
\int_{\Sigma} F \psi_{j} \cdot n e^{-\frac{2}{h} f}=\int_{\Sigma} F \widetilde{\psi}_{j} \cdot n e^{-\frac{2}{h} f}+O\left(e^{-\frac{1}{h}\left(\min _{\partial \Omega} f+c\right)}\right) .
$$

The statement of Proposition 65 is then a straightforward consequence of Proposition 46. 
We are now in position to prove Theorem 5 .

Proof of Theorem 5. Let us assume that (A0), (A1j), (A2j), and (A3j) hold. Recall that in this case, for all $x \in \mathrm{U}_{0}^{\Omega} \backslash\left\{x_{1}\right\}$, one has $f(\mathbf{j}(x))-f(x)<f\left(\mathbf{j}\left(x_{1}\right)\right)-f\left(x_{1}\right)$ and $\mathbf{j}\left(x_{1}\right) \cap \partial \Omega=\partial \mathrm{C}_{1} \cap \partial \Omega=\left\{z_{1}, \ldots, z_{\mathrm{k}_{1} \partial \mathrm{c}_{1}}\right\} \subset$ $\arg \min _{\partial \Omega} f \cap \mathrm{U}_{1}^{\partial \Omega}$. Moreover, from (55), it holds $x_{1} \in \arg \min _{\mathrm{C}_{1}} f=\arg \min _{\Omega} f=\arg \min \bar{\Omega} f$. Thus, one has

$$
f\left(\mathbf{j}\left(x_{1}\right)\right)=\min _{\partial \Omega} f \text { and } f\left(x_{1}\right)=\min _{\bar{\Omega}} f .
$$

Let us now consider $F \in L^{\infty}(\partial \Omega, \mathbb{R})$ and an open subset $\Sigma$ of $\partial \Omega$. First, since $\left\{\psi_{j}, j=1, \ldots, \mathrm{m}_{1}^{\bar{\Omega}}\right\}$ is an orthonormal basis of $\operatorname{Ran} \pi_{h}^{(1)}$ and $\nabla u_{h} \in \operatorname{Ran} \pi_{h}^{(1)}$, one has the following decomposition:

$$
\int_{\Sigma} F \partial_{n} u_{h} e^{-\frac{2}{h} f}=\sum_{j=1}^{\mathrm{m}_{1}^{\bar{\Omega}}}\left\langle\nabla u_{h}, \psi_{j}\right\rangle_{L_{w}^{2}} \int_{\Sigma} F \psi_{j} \cdot n e^{-\frac{2}{h} f} .
$$

Using in addition Corollary 64, Proposition 65, and (202), there exists $c>0$ such that for all $h>0$ small enough,

$$
\begin{aligned}
\int_{\Sigma} F \partial_{n} u_{h} e^{-\frac{2}{h} f}= & \sum_{j=1}^{\mathrm{k}_{1}^{\partial \Omega}}\left\langle\nabla u_{h}, \psi_{j}\right\rangle_{L_{w}^{2}} \int_{\Sigma} F \psi_{j} \cdot n e^{-\frac{2}{h} f} \\
& +\sum_{j=\mathrm{k}_{1}^{\partial \Omega}+1}^{\mathrm{m}_{1}^{\bar{\Omega}}} O\left(h^{-\frac{1}{2}} e^{-\frac{1}{h}\left(\min _{\partial \Omega} f-\min _{\bar{\Omega}} f\right)}\right) O\left(e^{-\frac{1}{h}\left(\min _{\partial \Omega} f+c\right)}\right) .
\end{aligned}
$$

Hence, when $\bar{\Sigma}$ does not contain any of the $z_{i}, i \in\left\{1, \ldots, \mathrm{k}_{1}^{\partial \Omega}\right\}$, from (203), Corollary 64, Proposition 65, and (202), one deduces the following relation for some $c>0$ independent of $h$ and every $h>0$ small enough:

$$
\begin{aligned}
\int_{\Sigma} F \partial_{n} u_{h} e^{-\frac{2}{h} f}= & \sum_{j=1}^{k_{1}^{\partial \Omega}} O\left(h^{-\frac{3}{4}} e^{-\frac{1}{h}\left(\min _{\partial \Omega} f-\min _{\bar{\Omega}} f\right)}\right) O\left(e^{-\frac{1}{h}\left(\min _{\partial \Omega} f+c\right)}\right) \\
& +O\left(e^{-\frac{1}{h}\left(2 \min _{\partial \Omega} f-\min _{\bar{\Omega}} f+c\right)}\right) \\
= & O\left(e^{-\frac{1}{h}\left(2 \min _{\partial \Omega} f-\min _{\bar{\Omega}} f+\frac{c}{2}\right)}\right) .
\end{aligned}
$$

This proves item $(i)$ in Theorem 5 .

Assume now that $\bar{\Sigma}$ does not contain any of the $z_{i}, i \in\left\{1, \ldots, \mathrm{k}_{1}^{\partial \mathrm{C}_{1}}\right\}$. Then, from (203), Corollary 64 , Proposition 65, and (202), one deduces that in the limit $h \rightarrow 0$ :

$$
\begin{aligned}
\int_{\Sigma} F \partial_{n} u_{h} e^{-\frac{2}{h} f}= & \sum_{j=1}^{\mathrm{k}_{1}^{\partial \mathrm{C}_{1}}} O\left(h^{-\frac{3}{4}} e^{-\frac{1}{h}\left(\min _{\partial \Omega} f-\min _{\bar{\Omega}} f\right)}\right) O\left(e^{-\frac{1}{h}\left(\min _{\partial \Omega} f+c\right)}\right) \\
& +\sum_{j=\mathrm{k}_{1}^{\partial \mathrm{C}_{1}}+1}^{\mathrm{k}_{1}^{\partial \Omega}} O\left(h^{-\frac{3}{4}} e^{-\frac{1}{h}\left(\min _{\partial \Omega} f-\min _{\bar{\Omega}} f\right)} \sqrt{\varepsilon_{h}}\right) O\left(h^{\frac{d-3}{4}} e^{-\frac{1}{h} \min _{\partial \Omega} f}\right) \\
& +O\left(e^{-\frac{1}{h}\left(2 \min _{\partial \Omega} f-\min _{\bar{\Omega}} f+c\right)}\right) \\
= & O\left(e^{-\frac{1}{h}\left(2 \min _{\partial \Omega} f-\min _{\bar{\Omega}} f+\frac{c}{2}\right)}\right)+O\left(h^{\frac{d-6}{4}} e^{-\frac{1}{h}\left(2 \min _{\partial \Omega} f-\min _{\bar{\Omega}} f\right)} \sqrt{\varepsilon_{h}}\right),
\end{aligned}
$$

where the constant $c>0$ is independent of $h$ and $\varepsilon_{h}$ satisfies (180). This proves item (ii) in Theorem 5 . 
Assume lastly that $\bar{\Sigma} \cap\left\{z_{1}, \ldots, z_{\mathrm{k}_{1}^{\partial \mathrm{c}_{1}}}\right\}=\left\{z_{i}\right\}, F$ is $C^{\infty}$ in a neighborhood of $z_{i}$, and $z_{i} \in \Sigma$. From (203), Corollary 64, Proposition 65, and (202), one then deduces that in the limit $h \rightarrow 0$, it holds for some $c>0$ and $\varepsilon_{h}$ which satisfies (180),

$$
\begin{aligned}
\int_{\Sigma} F \partial_{n} u_{h} e^{-\frac{2}{h} f} & =\left\langle\nabla u_{h}, \psi_{i}\right\rangle_{L_{w}^{2}} \int_{\Sigma} F \psi_{i} \cdot n e^{-\frac{2}{h} f}+O\left(h^{\frac{d-6}{4}} e^{-\frac{1}{h}\left(2 \min _{\partial \Omega} f-\min _{\bar{\Omega}} f\right)} \sqrt{\varepsilon_{h}}\right) \\
& =-B_{i} C_{i, 1} h^{\frac{d-6}{4}} e^{-\frac{1}{h}\left(2 \min _{\partial \Omega} f-\min _{\bar{\Omega}} f\right)}\left(F\left(z_{i}\right)+O\left(\sqrt{\varepsilon_{h}}\right)+O(h)\right),
\end{aligned}
$$

where the constants $B_{i}$ and $C_{i, 1}$ are defined in (117)-(106). This concludes the proof of item (iii) in Theorem 5 .

\subsection{Proof of Theorem 1}

The proof of Theorem 1 is a straightforward consequence of Theorem 2, Proposition 58 and Theorem 5. Indeed, let us recall that from (12), one has:

$$
\mathbb{E}_{\nu_{h}}\left[F\left(X_{\tau_{\Omega}}\right)\right]=-\frac{h}{2 \lambda_{h}} \frac{\int_{\Omega} F \partial_{n} u_{h} e^{-\frac{2}{h} f}}{\int_{\Omega} u_{h} e^{-\frac{2}{h} f}} .
$$

Moreover, recall that (A1), (A2), and (A3) (see Section 2.4 and more precisely Lemma 21) are equivalent to the assumptions $(\mathbf{A} 1 \mathbf{j}),(\mathbf{A} 2 \mathbf{j})$, and $(\mathbf{A} 3 \mathbf{j})$. In addition, under $(\mathbf{A} 1 \mathbf{j})$, one has $\mathbf{C}_{1}=\mathrm{C}_{\max }($ see Lemma 21$), \mathrm{k}_{1}^{\partial C_{1}}=$ $\mathrm{k}_{1}^{\partial \mathrm{C}_{\max }}($ see $(54)), f\left(\mathbf{j}\left(x_{1}\right)\right)=\min _{\partial \Omega} f\left(\right.$ see $(\mathbf{A} 3 \mathbf{j})$ together with the fact that $\left.\mathbf{j}\left(x_{1}\right) \subset \partial \mathrm{C}_{1}\right)$ and $f\left(x_{1}\right)=$ $\min _{\bar{\Omega}} f$ (see (55)). Thus, injecting the results of Theorem 2 (and more precisely (105)), Proposition 58 (applied to $\mathrm{O}=\Omega$, see (179)) and Theorem 5 in (204), one obtains the statements of Theorem 1.

\section{Main notation used in this work}

- $\tau_{\Omega}$, Equation (2)

- $L_{f, h}^{(0)}, L_{f, h}^{D,(0)}$, Equation (7) and Proposition 4

- $\lambda_{h}, u_{h}, \nu_{h}$, Equations (8)-(9), (10)-(11)

- Assumptions (A0), (A1), (A2), (A3), and (A4), Section 1.3.1

- $\{f<a\},\{f \leq a\},\{f=a\}$, Section 1.3.1

- $\mathrm{H}_{f}(x)$, Equation (13)

- $\mathrm{C}_{\max }$, Section 1.3.1

- $\mathcal{C}, \mathrm{C}(x)$, Equations (14)-(15) and (32)

- $\mathrm{U}_{0}^{\Omega}=\left\{x_{1}, \ldots, x_{\mathrm{m}_{0}^{\Omega}}\right\}$ and $\mathrm{m}_{0}^{\Omega}$, Equation (16)

- $\mathrm{U}_{1}^{\Omega}=\left\{z_{\mathrm{m}_{1}^{\partial \Omega}+1}, \ldots, z_{\mathrm{m}_{1}}\right\}$ and $\mathrm{m}_{1}^{\Omega}$, Equation (21)

- $\mathrm{U}_{1}^{\partial \Omega}=\left\{z_{1}, \ldots, z_{\mathrm{m}_{1}^{\partial \Omega}}\right\}$ and $\mathrm{m}_{1}^{\partial \Omega}$, Equations (17)-(18) and (20)

- $\mathrm{U}_{1}^{\bar{\Omega}}=\left\{z_{1}, \ldots, z_{\mathrm{m}_{1}^{\partial \Omega}}, z_{\mathrm{m}_{1}^{\partial \Omega}+1}, \ldots, z_{\mathrm{m}_{1}^{\bar{\Omega}}}\right\}$ and $\mathrm{m}_{1}^{\bar{\Omega}}$, Equation (19)

- $\left\{z_{1}, \ldots, z_{\mathbf{k}_{1}^{\partial \Omega}}\right\}$ and $\mathbf{k}_{1}^{\partial \Omega}$, Equation (22)

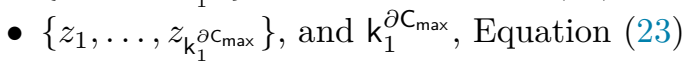

- $a_{i}$, Equation (27)

- $\mathrm{C}(\lambda, x), \mathrm{C}^{+}(\lambda, x), \lambda(x)$, Definition 10

- $\mathrm{N}_{1},\left(C_{\ell}\right)_{\ell \in\left\{1, \ldots, \mathrm{N}_{1}\right\}}=\left(\mathrm{E}_{1, \ell}\right)_{\ell \in\left\{1, \ldots, \mathrm{N}_{1}\right\}}$, Definition 12

- $\mathrm{U}_{1}^{\text {ssp }}, \mathcal{C}_{\text {crit }}$, Definition 13

- $\mathbf{j}, \widetilde{\mathbf{j}},\left(\mathrm{N}_{k}\right)_{k \geq 2},\left(x_{k, \ell}\right)_{k \geq 1, \ell \in\left\{1, \ldots, \mathrm{N}_{k}\right\}}$,

$\left(\mathrm{E}_{k, \ell}\right)_{k \geq 2, \ell \in\left\{1, \ldots, \mathrm{N}_{k}\right\}}$, Section 2.3

- $\Lambda^{p} C^{\infty}(\bar{\Omega}), \quad \Lambda^{p} C_{T}^{\infty}(\bar{\Omega}), \quad \Lambda^{p} L_{w}^{2}(\Omega), \quad \Lambda^{p} H_{w}^{q}(\Omega)$, $\Lambda^{p} H_{w, T}^{q}(\Omega), \quad \Lambda^{p} L^{2}(\Omega), \quad \Lambda^{p} H^{q}(\Omega), \quad \Lambda^{p} H_{T}^{q}(\Omega)$, $\Lambda^{p} H_{N}^{q}(\Omega)$, Section 3.1.1

- $\|\cdot\|_{H_{w}^{q}},\langle,\rangle_{L_{w}^{2}},\|\cdot\|_{H^{q}},\langle,\rangle_{L^{2}}$, Section 3.1.1

- $\Delta_{f, h}^{(p)}, \Delta_{f, h}^{D,(p)}(\Omega), L_{f, h}^{D,(p)}(\Omega)$, Section 3.1.2

- $\pi_{E}$, Lemma 25

- $\pi_{h}^{(p)}$, Equation (60)

- $\widetilde{v}_{k, \ell}$ and $\chi_{k, \ell}$, Definition 26

- $\Phi_{j}$, Equations (73) and (87)

- $w_{j}$, Propositions 30 and 35

- $\theta_{j}$ and $\widetilde{\phi}_{j}$, Definitions 31 and 36 
- $u_{j, w k b}^{(1)}, c_{j}(h)$, Propositions 33 and 38

- $\widetilde{\phi}_{j, w k b}$, Equation (99)

- $\left(\mathrm{E}_{k}\right)_{k \in\left\{1, \ldots, \mathrm{m}_{0}^{\Omega}\right\}},\left(\chi_{k}^{\varepsilon, \varepsilon_{1}}\right)_{k \in\left\{1, \ldots, \mathrm{m}_{0}^{\Omega}\right\}},\left(\widetilde{v}_{k}\right)_{k \in\left\{1, \ldots, \mathbf{m}_{0}^{\Omega}\right\}}$, $\left(x_{k}\right)_{k \in\left\{1, \ldots, \mathbf{m}_{0}^{\Omega}\right\}}$, Definition 41

- $\widetilde{u}_{k}$ and $\widetilde{\psi}_{j}$, Definition 42

- $\lambda_{2, h}$, Theorem 2

- $B_{j}$, Equation (106)

- $\varepsilon_{j, k}, p_{j, k}$ and $C_{j, k}$, Equations (115), (116), (117)

- $S=\left(S_{j, k}\right)$, Equation (126)

- $\widetilde{S}=\left(\widetilde{S}_{j, k}\right)$, Equation $(127)$
- $D, D_{k, k}$, and $q_{k}$, Equation (128)

- $\widetilde{C}=\left(\widetilde{C}_{j, k}\right)$, Equations (131)-(132)

- $\mathrm{S}_{k}$, Equation (146)

- $\eta_{i}(T)$, Lemma 52

- $C_{0}, C_{1}$, Equation (147)

- $\lambda_{k, h}$, Equation (150)

- $\varepsilon_{h}$, Equation (180)

- $\widetilde{\pi}_{h}^{(0)}$, Equation (185)

- $\kappa_{j i}$, Equation (196)

- $Z_{j}$ and $\psi_{j}$, Equation (197)

\section{Acknowledgements}

This work is supported by the European Research Council under the European Union's Seventh Framework Programme (FP/2007-2013)/ERC Grant Agreement number 614492. The authors thank Laurent Michel for fruitful discussions.

\section{References}

[1] G. Di Gesù, T. Lelièvre, D. Le Peutrec, B. Nectoux, Sharp asymptotics of the first exit point density, Ann. PDE 5 (1) (2019).

[2] G. Di Gesù, T. Lelièvre, D. Le Peutrec, B. Nectoux, Jump Markov models and transition state theory: the quasi-stationary distribution approach, Faraday Discuss. 195 (2017) 469-495.

[3] T. Lelièvre, Mathematical foundations of accelerated molecular dynamics methods, in: W. Andreoni, S. Yip (Eds.), Handbook of Materials Modeling, Springer, Cham, 2019.

[4] M.V. Day, Mathematical approaches to the problem of noise-induced exit, in: Stochastic Analysis, Control, Optimization and Applications, in: Systems Control Found. Appl., Birkhäuser, Boston, 1999, pp. 269-287.

[5] B. Matkowsky, Z. Schuss, The exit problem for randomly perturbed dynamical systems, SIAM J. Appl. Math. 33 (2) (1977) 365-382.

[6] T. Naeh, M. Klosek, B. Matkowsky, Z. Schuss, A direct approach to the exit problem, SIAM J. Appl. Math. 50 (2) (1990) 595-627.

[7] Z. Schuss, B. Matkowsky, The exit problem: a new approach to diffusion across potential barriers, SIAM J. Appl. Math. 36 (3) (1979) 604-623.

[8] S. Kamin, On elliptic singular perturbation problems with turning points, SIAM J. Math. Anal. 10 (3) (1979) $447-455$.

[9] S. Kamin, Elliptic perturbation of a 1st order operator with a singular point of attracting type, Indiana Univ. Math. J. 27 (6) (1978) 935-952.

[10] B. Perthame, Perturbed dynamical systems with an attracting singularity and weak viscosity limits in Hamilton-Jacobi equations, Trans. Am. Math. Soc. 317 (2) (1990) 723-748.

[11] H. Ishii, P. Souganidis, Metastability for parabolic equations with drift: part 1, Indiana Univ. Math. J. 64 (2015) 875-913.

[12] H. Ishii, P. Souganidis, Metastability for parabolic equations with drift, part II: the quasilinear case, Indiana Univ. Math. J. 66 (1) (2017) 315-360.

[13] M. Freidlin, A. Wentzell, Random Perturbations of Dynamical Systems, Springer-Verlag, 2012.

[14] M. Day, On the asymptotic relation between equilibrium density and exit measure in the exit problem, Stoch. Int. J. Probab. Stoch. Process. 12 (3-4) (1984) 303-330.

[15] M. Day, Recent progress on the small parameter exit problem, Stoch. Int. J. Probab. Stoch. Process. 20 (2) (1987) $121-150$.

[16] C. Le Bris, T. Lelièvre, M. Luskin, D. Perez, A mathematical formalization of the parallel replica dynamics, Monte Carlo Methods Appl. 18 (2) (2012) 119-146.

[17] T. Lelièvre, F. Nier, Low temperature asymptotics for quasistationary distributions in a bounded domain, Anal. PDE 8 (3) (2015) 561-628, https://doi.org/10.2140/apde.2015.8.561.

[18] T. Lelièvre, D. Le Peutrec, B. Nectoux, The exit from a metastable state: concentration of the exit point distribution on the low energy saddle points, part 2, 2019, in preparation.

[19] P. Collet, S. Martínez, J. San Martín, Quasi-Stationary Distributions: Markov Chains, Diffusions and Dynamical Systems, Springer Science \& Business Media, 2012.

[20] D. Gilbarg, N. Trudinger, Elliptic Partial Differential Equations of Second Order, Classics in Mathematics, Springer-Verlag, Berlin, 2001, reprint of the 1998 edition.

[21] L. Evans, Partial Differential Equations, 2nd edition, Graduate Studies in Mathematics, vol. 19, American Mathematical Society, Providence, 2010.

[22] J. Jost, Riemannian Geometry and Geometric Analysis, 7th ed., Springer, 2017.

[23] B. Helffer, F. Nier, Quantitative analysis of metastability in reversible diffusion processes via a Witten complex approach: the case with boundary, Mém. Soc. Math. Fr. 105 (2019). 
[24] G. Di Gesù, T. Lelièvre, D. Le Peutrec, B. Nectoux, The exit from a metastable state: concentration of the exit point distribution on the low energy saddle points, 2019, arXiv:1902.03270.

[25] T. Lelièvre, D. Le Peutrec, B. Nectoux, Exit event from a metastable state and Eyring-Kramers law for the overdamped Langevin dynamics, in: G. Giacomin, S. Olla, E. Saada, H. Spohn, G. Stoltz (Eds.), Stochastic Dynamics out of Equilibrium, in: Springer Proceedings in Mathematics \& Statistics, 2017, pp. 331-363, https://www.springer.com/gp/book/ 9783030150952 , Hal-01696187.

[26] D. Le Peutrec, B. Nectoux, Repartition of the quasi-stationary distribution and first exit point density for a double-well potential, arXiv:1902.06304, 2019.

[27] B. Nectoux, Analyse spectrale et analyse semi-classique pour la métastabilité en dynamique moléculaire, Ph.D. Thesis, Université Paris Est. Hal-01653731, 2017.

[28] F. Hérau, M. Hitrik, J. Sjöstrand, Tunnel effect and symmetries for Kramers-Fokker-Planck type operators, J. Inst. Math. Jussieu 10 (3) (2011) 567-634, https://doi.org/10.1017/S1474748011000028.

[29] A. Bovier, M. Eckhoff, V. Gayrard, M. Klein, Metastability in reversible diffusion processes, I: sharp asymptotics for capacities and exit times, J. Eur. Math. Soc. 6 (4) (2004) 399-424, https://doi.org/10.4171/JEMS/14.

[30] A. Bovier, V. Gayrard, M. Klein, Metastability in reversible diffusion processes, II: precise asymptotics for small eigenvalues, J. Eur. Math. Soc. 7 (1) (2005) 69-99, https://doi.org/10.4171/JEMS/22.

[31] B. Helffer, M. Klein, F. Nier, Quantitative analysis of metastability in reversible diffusion processes via a Witten complex approach, Mat. Contemp. 26 (2004) 41-85.

[32] B. Helffer, J. Sjöstrand, Puits multiples en meécanique semi-classique IV Etude du complexe de Witten, Commun. Partial Differ. Equ. 10 (3) (1985) 245-340.

[33] G. Schwarz, Hodge Decomposition-A Method for Solving Boundary Value Problems, Lecture Notes in Mathematics, vol. 1607, Springer-Verlag, Berlin, 1995.

[34] E. Witten, Supersymmetry and Morse theory, J. Differ. Geom. 17 (4) (1982) 661-692, http://projecteuclid.org/euclid.jdg/ 1214437492.

[35] B. Helffer, H. Kovař́k, M.P. Sundqvist, On the semiclassical analysis of the ground state energy of the Dirichlet Pauli operator III: magnetic fields that change sign, Lett. Math. Phys. 109 (7) (2019) 1533-1558.

[36] B. Helffer, Spectral Theory and Its Applications, vol. 139, Cambridge University Press, 2013.

[37] D. Le Peutrec, Small eigenvalues of the Neumann realization of the semiclassical Witten Laplacian, Ann. Fac. Sci. Toulouse Math. (6) 19 (3-4) (2010) 735-809, http://afst.cedram.org/item?id=AFST_2010__19_3-4_735_0.

[38] L. Michel, About small eigenvalues of Witten Laplacian, Pure Appl. Anal. 1 (2) (2019) 149-206.

[39] C. Landim, M. Mariani, I. Seo, Dirichlet's and Thomson's principles for non-selfadjoint elliptic operators with application to non-reversible metastable diffusion processes, Arch. Ration. Mech. Anal. 231 (2) (2019) 887-938.

[40] R.A. Holley, S. Kusuoka, D. Stroock, Asymptotics of the spectral gap with applications to the theory of simulated annealing, J. Funct. Anal. 83 (2) (1989) 333-347.

[41] L. Miclo, Comportement de spectres d'opérateurs de Schrödinger à basse température, Bull. Sci. Math. 119 (6) (1995) 529-554.

[42] B. Simon, Trace Ideals and Their Applications, vol. 35, Cambridge University Press, Cambridge, 1979.

[43] D. Le Peutrec, Small singular values of an extracted matrix of a Witten complex, CUBO, Math. J. 11 (4) (2009) 49-57. 